\title{
Molecular Structure of Pyrazinamide: a Critical Assessment of Modern Gas Electron Diffraction Data from Three Laboratories
}

\author{
Arseniy A. Otlyotov* Georgiy V. Girichev* Anatolii N. Rykov ${ }^{\dagger} \quad$ Timo Glodde $^{\ddagger}$ \\ Yury V. Vishnevskiy ${ }^{\S}$
}

\section{Abstract}

Accuracy and precision of molecular parameters determined by modern gas electron diffraction method have been investigated. Diffraction patterns of gaseous pyrazinamide have been measured independently in three laboratories, in Bielefeld (Germany), Ivanovo (Russia) and Moscow (Russia). All data sets have been analysed in equal manner using highly controlled background elimination procedure and flexible restraints in molecular structure refinement. In detailed examination and comparison of the obtained results we have determined the average experimental precision of $0.004 \AA$ for bond lengths and 0.2 degrees for angles. The corresponding average deviations of the refined parameters from the ae-CCSD $(\mathrm{T}) / \mathrm{cc}-$ pwCVTZ theoretical values were $0.003 \AA$ and 0.2 degrees. The average precision for refined amplitudes of interatomic vibrations was determined to be $0.005 \AA$. It is recommended to take into account these values in calculations of total errors for refined parameters of other molecules with comparable complexity.

\footnotetext{
*Ivanovo State University of Chemistry and Technology, Department of Physics, Sheremetievskiy ave., 7, 153000 Ivanovo, Russian Federation

${ }^{\dagger}$ M. V. Lomonosov Moscow State University, Faculty of Chemistry, Department of Physical Chemistry, GSP-1, 1-3 Leninskiye Gory, 119991 Moscow, Russian Federation

${ }^{\ddagger}$ Lehrstuhl für Anorganische Chemie und Strukturchemie, Universität Bielefeld, Universitätsstraße 25, 33615 Bielefeld, Germany. Tel.: +49521106 6164 Fax: +49521 1066026

§Corresponding author, yury.vishnevskiy@uni-bielefeld.de
}

\section{Introduction}

Gas electron diffraction (GED) is one of the most well established direct methods for the experimental investigation of molecular structure in the gas phase. Already in 1930 due to GED appeared data on molecular structures of carbon tetrachloride, cyclopentane and cyclohexane, benzene and some of its chloro derivatives [1], although with very limited accuracy and precision. Since then the GED method improved significantly and its accuracy increased due to developments both in experimental techniques and in methods of data interpretation, see [2] for a review. Still, the investigation of fine structural effects can be a big challenge, especially in cases of large molecules and complicated vapor compositions. Different GED groups have elaborated approaches for solving these problems. However, each laboratory has its own unique experimental setup. In addition, methods for data reduction and structure refinement can also differ. In this respect an important question arises about reproducibility of results produced in different groups. It is considered to be normal when molecular structures from modern investigations deviate from those of significantly older studies, for example see the case of antimony(III) oxide [3]. This can be explained by development of the method. A completely different situation is when results of two or more concurrent investigations disagree. Several publications in the past have been addressed to this problem [4-10]. Some of them documented significant deviations in structural parameters $[4,6,10]$, which was an indication of relatively large systematic errors leading to biased parameters or underes- 
timated uncertainties. The work of Campanelli et al. [10] should be mentioned due to careful comparison of molecular structures determined by GED method in different groups for a series of halogenated benzene derivatives. An expressive example from this paper is the $r_{\mathrm{g}}$ length of $\mathrm{C}-\mathrm{C}$ bonds in benzene ring of 1,3,5-trichlorobenzene determined to be $1.392(2)$ $\AA$ [11] and 1.400(1) $\AA$ [12]. The difference of $0.008 \AA$ between the values from the two independent investigations is considered to be significant taking into account the symmetry of the molecule and the high precision typical for benzene bonds, as uncertainties suggest.

The development of the GED method in the last two decades requires a thorough assessment of data and refined molecular structures on modern level. In this work we have analysed three data sets measured independently in laboratories at Bielefeld University (denoted below as UBi), M. V. Lomonosov Moscow State University (LMSU) and Ivanovo State University of Chemistry and Technology (ISUCT). As a test molecule we chose pyrazinamide (PZA, see Figure 1). This compound has been recently investigated by the GED method [13] and the obtained experience showed its suitability for our study. Here the most relevant properties of PZA are (a) the stability under the experimental conditions, (b) the existence in only one single conformation in a broad range of temperatures, (c) a reasonable complexity of its geometrical structure. The last two properties ensure that the refinement of the molecular structure is not an overcomplicated problem. On the other hand, the structure is complex enough to make it possible an overinterpretation of experimental data. Thus can be obtained valuable information on the accuracy and precision of molecular structures obtained by the modern GED method.

\section{$3 \quad$ Experiments}

Measurements of electron diffraction patterns of gaseous PZA have been performed independently in three laboratories. Details of the experiments are given below. A commercial sample of PZA (Acros Organics, purity at least $99 \%$ ) was used from the

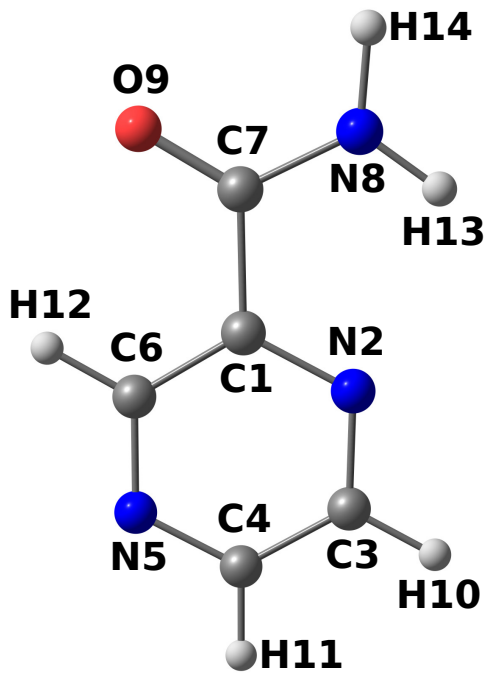

Figure 1: Molecular structure of pyrazinamide with atom numbering.

same batch in all cases. Note, different temperatures in experiments were required due to construction peculiarities of particular electron diffraction units.

\section{$3.1 \mathrm{UBi}$}

At Bielefeld University diffraction patterns of PZA have been measured at $443-448 \mathrm{~K}$ on BAS-MP Imaging Plates in Balzers Eldigraph KD-G2 diffractometer, which has been significantly modified [14] and also improved recently [15]. Gaseous $\mathrm{CCl}_{4}$ was used as standard, for which diffraction patterns were also measured along with the studied substance. The plates were scanned using a calibrated Fuji BAS1800II reader. As usually, two series of measurements were done, one for middle camera $(250 \mathrm{~mm})$ and another for the long camera setting $(500 \mathrm{~mm})$ to obtain data for the widest possible range of scattering angles. Details of experimental conditions are provided in Table S1 of Supporting Information. The data reduction for all patterns has been done in the same way according to our standard procedure [16]. Electron wavelengths were refined from the obtained intensity functions of $\mathrm{CCl}_{4}$ as usually [17] taking the most accurate available parameters [18]. 


\section{$3.2 \quad$ LMSU}

At M. V. Lomonosov Moscow State University electron diffraction patterns of PZA have been measured at 400-404 K with EG-100M apparatus (for details see Table S2 in SI). Photo films MACO EM-FILM EMS ES209 were used for recording diffraction patterns. The measurements were done for two camera settings, long $(362.3 \mathrm{~mm})$ and short $(193.9 \mathrm{~mm})$. Diffraction patterns of gaseous $\mathrm{CCl}_{4}$ have been measured in the same experiments for calibration of electron wavelengths. Exposed photo films were scanned on a EPSON Perfection V850 Pro scanner, which had been beforehand calibrated for optical density and spatial resolution. The scanning mode was 16bit grayscale 800 dpi. Data reduction of the obtained digitized images and refinement of electron wavelengths were done in the same way as for UBi data.

\section{$3.3 \quad$ ISUCT}

At Ivanovo State University of Chemistry and Technology synchronous gas electron diffraction and mass spectrometric (GED/MS) experiments were carried out using EMR-100/APDM-1 unit [19-21] for long (LD) and short (SD) nozzle-to-film distances. A sample of PZA was evaporated from a stainless steel (X18H10T) effusion cell with a cylindrical effusion nozzle of $0.5 \times 1.6 \mathrm{~mm}$ size (diameter x length) at $372(5)$ K. Main conditions of the experiments are listed in Table S3 of SI. Two additional films for polycrystalline $\mathrm{ZnO}$ were recorded before and after taking the diffraction patterns of PZA in order to determine accurate electron wavelengths. Optical densities of the diffraction patterns were measured by a modified MD-100 (Carl Zeiss, Jena) microdensitometer [22] with a step size of $0.1 \mathrm{~mm}$ along diagonal. A $10 \times 130 \mathrm{~mm}$ region was scanned; the number of equidistant scan lines was 33 .

\section{Structure refinement}

A complete and unambiguous refinement of molecular structure for PZA solely from gas-phase electron diffraction data is impossible. Therefore a series of quantum-chemical calculations were done, whose results were then used in the analysis of the measured data. First, optimization of molecular structure has been done at ae-CCSD (T)/cc-pwCVTZ level of theory as implemented in the Cfour package of programs [23]. The obtained theoretical parameters were used in refinement as starting approximation and for regularization as flexible restraints. For the description of this method see [24] and references therein. The refinements were done using the UNEX program [25] assuming $C_{\mathrm{s}}$ symmetry, since the planarity of the structure was previously confirmed both experimentally [13] and theoretically [26]. Special attention has been paid to the generalized regularization factor $\alpha$ (see Eq. 3 in [24]). It has been adjusted manually to keep the balance of maximized contributions of experimental data into refined parameters and stabilized solution of the least-squares problem. For this the deviations of refined values of parameters from their initial values have been analysed for different $\alpha$, see Tables S7-S12 of SI. Also, least-squares correlation factors between parameters were taken into account; their values in final refinements were less then 0.6. Contributions of GED data into refined parameters were calculated according to W2 method [27], their values are provided in Table S6 of SI.

As with the molecular structure supplementary information was also required for vibrational parameters of interatomic pairs in PZA for interpretation of the experimental electron diffraction intensities. For this, calculations of anharmonic force fields and frequencies were done using a series of DFT functionals (B3LYP, O3LYP, X3LYP, PW6B95, PBE0, B3PW91, TPSSh) paired with def2-TZVP basis set as implemented in the Gaussian program package [28]. The calculated frequencies were compared with available experimental values obtained for isolated PZA monomer in xenon matrix [26], see Table S13. Several functionals gave low root-mean-square deviations and finally the results of B3LYP calculation have been taken for further processing. Optimized at this level of theory geometry and calculated analytic harmonic and numeric cubic force fields were used in computation of vibrational amplitudes $l$ and corrections $\left(r_{\mathrm{e}}-r_{\mathrm{a}}\right)$ for interatomic pairs of PZA. This has been done using the VibModule program [29]. Thus, 
the molecular structure of PZA has been refined in terms of equilibrium geometry. In addition, in the least squares analysis of all data sets scale factors for groups of amplitudes were refined in the same manner. The grouping scheme, the theoretical and refined $l$ values as well as corrections are provided in Tables S14-S16.

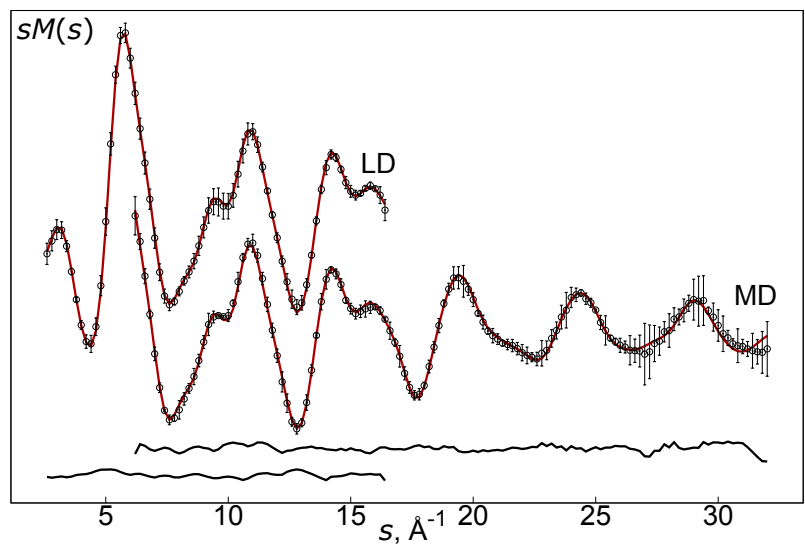

Figure 2: Molecular intensity functions measured at UBi (dots), corresponding model (lines) and difference curves. Vertical bars indicate threefold standard deviations.

Special attention has been focused on the quality of background lines calculated for the measured intensity functions. Any nonsmoothness on background can significantly influence the resulted values of refined parameters [30]. Poorly levelled total intensity functions are prone to this problem [18]. Therefore backgrounds were approximated with cubic splines for reduced experimental intensity functions (see description of the method and discussion in [18]) measured at UBi and ISUCT. For the data set from ISUCT this procedure was of critical importance due to the special form of the sector device, which resulted in large drops of intensity values in a very narrow range of diffraction angles. The data set from LMSU was already well levelled and did not require this kind of treatment. Thus, for all data sets equally strong criteria have been applied in calculating background lines. Their smoothness has been controlled by defining the maximal allowed numbers of inflec-

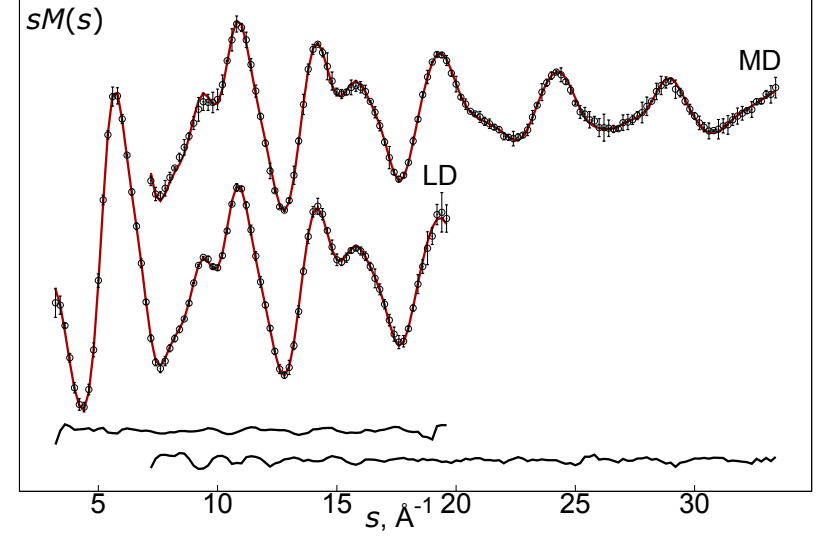

Figure 3: Molecular intensity functions measured at LMSU (dots), corresponding model (lines) and difference curves. Vertical bars indicate threefold standard deviations.

tion points (see Tables S1-S3 of SI).

In the least-squares analysis averaged intensity functions were used in two variants. First, the averaging of total intensities has been tested. The obtained curves have been converted into experimental molecular intensity $s M(s)$ functions by applying multiplicative background correction as described above. However, in this procedure could not be obtained reliable standard deviations for the $s M(s)$ values. In the other variant all individual total intensities were first converted into $s M(s)$ functions, which were then averaged. In this case realistic standard deviations $\sigma$ were obtained and used in least squares analysis for calculation of weighting factors as $w=1 / \sigma^{2}$. Finally this variant has been accepted as the main. Results of refinements of both types are provided in Table S5. Molecular intensity functions from the main refinements are shown in Figures 2, 3, 4. For radial distribution functions see Figure 5.

\section{Results}

Before analyzing results several statements should be made about design of our study. In comparison to previous work this investigation has advantages in the following aspects. 


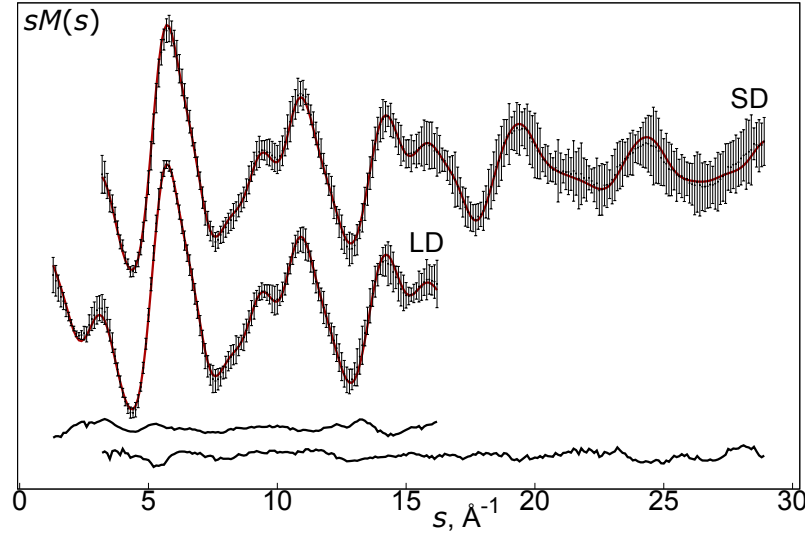

Figure 4: Molecular intensity functions measured at ISUCT (dots), corresponding model (lines) and difference curves. Vertical bars indicate threefold standard deviations.

- The refinements of the molecular structures were performed in terms of equilibrium parameters. Therefore their comparison does not require considering differences between temperatures in the experiments. In addition, experimental conditions can differ in extents of the spread of the diffraction volume. As the result the refined effective values of amplitudes can also differ from one set of the data to another due to the effect of finite sample size $[31,32]$. Because of this problem parameters of $r_{\mathrm{g}}$ type (amplitudes are required for their calculation) cannot be used for accurate comparison.

- In this work we used flexible restraints to stabilize solutions of the least squares problem. We explicitly avoided fixing any geometrical parameters as this would blur the experimental status of the refined structures. On the other hand for the case of flexible restraints recently it has become possible to calculate contributions of experimental data into refined parameters $[13,27]$. This has been utilized for PZA to maximize the contribution of the GED data in the refined structures and to keep the solution of the inverse problem stable. Within this procedure were calculated pure experimental standard deviations

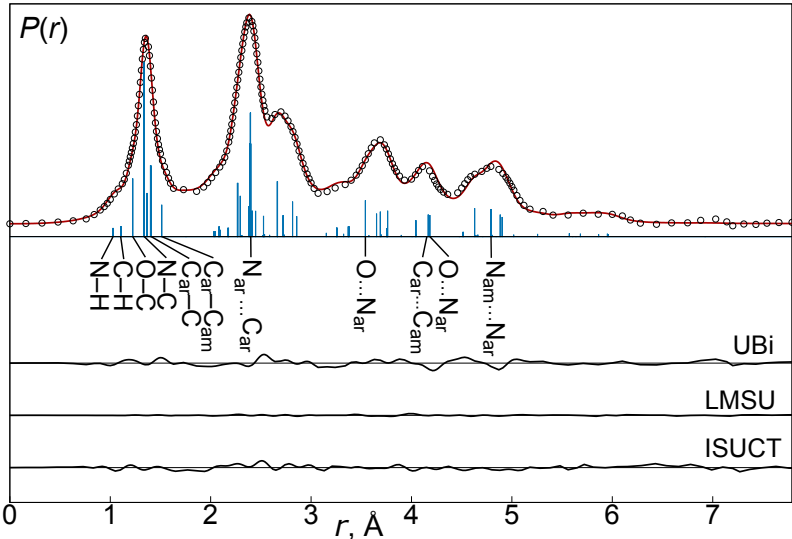

Figure 5: Experimental (dots) and model (line) radial distribution functions and their differences. Vertical bars correspond to interatomic distances in PZA.

for the refined parameters as described in [13]. These values allowed more correct comparison of the results.

- In the refinements we used controlled background lines with very high requirements for their smoothness. This principle was applied to all data sets from each laboratory. In this way the influence of background on the refined structures has been minimized.

Selected calculated and refined geometrical parameters of PZA are collected in Table 1. The complete set of parameters can be found in Table S4 of SI. Based on these data weighted root-mean-square deviations (WRMSD) have been calculated for all pairs of parameter sets as

$$
\mathrm{WRMSD}=\sqrt{\frac{\sum_{i=1}^{N} w_{i}\left(p_{i}^{\mathrm{A}}-p_{i}^{\mathrm{B}}\right)^{2}}{\sum_{i=1}^{N} w_{i}}}
$$

where $p_{i}^{\mathrm{A}}$ and $p_{i}^{\mathrm{B}}$ are the values of the $i$-th parameter from the sets $\mathrm{A}$ and $\mathrm{B}$ (one of $\mathrm{CCSD}(\mathrm{T})$, UBi, LMSU or ISUCT), respectively; $w_{i}$ is the weighting factor; $N$ is the total number of parameters. The weighting factors were calculated from respective experimental standard deviations of parameters (first values in 
Table 1: Selected theoretical and experimental structural parameters of $\mathrm{PZA}^{\mathrm{a}}$

\begin{tabular}{llllll}
\hline \multirow{2}{*}{ Method } & \multirow{2}{*}{ CCSD(T) } & \multicolumn{4}{c}{ GED $^{\mathrm{b}}$} \\
\cline { 3 - 6 } Parameter & & UBi & LMSU & ISUCT & $\Delta_{\max }{ }^{\mathrm{c}}$ \\
\hline$r(\mathrm{C} 1-\mathrm{N} 2)$ & 1.337 & $1.333(3 / 2)$ & $1.339(1 / 1)$ & $1.335(4 / 4)$ & 0.006 \\
$r(\mathrm{C} 1-\mathrm{C} 6)$ & 1.394 & $1.398(3 / 2)$ & $1.398(2 / 1)$ & $1.392(4 / 4)$ & 0.006 \\
$r(\mathrm{C} 1-\mathrm{C} 7)$ & 1.505 & $1.501(2 / 2)$ & $1.506(1 / 1)$ & $1.497(3 / 3)$ & 0.009 \\
$r(\mathrm{~N} 2-\mathrm{C} 3)$ & 1.335 & $1.329(3 / 3)$ & $1.337(2 / 1)$ & $1.330(4 / 4)$ & 0.008 \\
$r(\mathrm{C} 3-\mathrm{C} 4)$ & 1.393 & $1.391(3 / 2)$ & $1.397(2 / 1)$ & $1.391(4 / 4)$ & 0.006 \\
$r(\mathrm{C}-\mathrm{H})_{\text {average }}$ & 1.082 & $1.083(11 / 3)$ & $1.081(11 / 2)$ & $1.085(14 / 4)$ & 0.004 \\
$r(\mathrm{C} 4-\mathrm{N} 5)$ & 1.337 & $1.333(3 / 3)$ & $1.338(2 / 1)$ & $1.334(4 / 4)$ & 0.005 \\
$r(\mathrm{~N} 5-\mathrm{C} 6)$ & 1.336 & $1.333(3 / 3)$ & $1.338(2 / 1)$ & $1.330(4 / 4)$ & 0.008 \\
$r(\mathrm{C} 7-\mathrm{N} 8)$ & 1.350 & $1.347(3 / 3)$ & $1.352(2 / 1)$ & $1.348(4 / 4)$ & 0.005 \\
$r(\mathrm{C} 7-\mathrm{O} 9)$ & 1.219 & $1.220(2 / 2)$ & $1.221(1 / 1)$ & $1.214(2 / 2)$ & 0.007 \\
$r(\mathrm{~N}-\mathrm{H})_{\text {average }}$ & 1.003 & $1.005(11 / 3)$ & $1.003(7 / 2)$ & $1.001(14 / 4)$ & 0.004 \\
$\angle(\mathrm{N} 2-\mathrm{C} 1-\mathrm{C} 6)$ & 122.2 & $121.8(4 / 3)$ & $122.0(2 / 1)$ & $121.8(4 / 4)$ & 0.2 \\
$\angle(\mathrm{C} 1-\mathrm{N} 2-\mathrm{C} 3)$ & 115.9 & $116.0(2 / 1)$ & $115.9(1 / 1)$ & $116.0(3 / 2)$ & 0.1 \\
$\angle(\mathrm{C} 6-\mathrm{C} 1-\mathrm{C} 7)$ & 119.3 & $119.9(3 / 2)$ & $119.6(2 / 2)$ & $119.5(5 / 4)$ & 0.4 \\
$\angle(\mathrm{C} 1-\mathrm{C} 6-\mathrm{N} 5)$ & 122.0 & $122.2(5 / 3)$ & $122.3(3 / 2)$ & $122.5(8 / 5)$ & 0.3 \\
$\angle(\mathrm{C} 1-\mathrm{C} 7-\mathrm{N} 8)$ & 113.8 & $113.7(3 / 2)$ & $113.8(2 / 1)$ & $114.2(3 / 2)$ & 0.5 \\
$\angle(\mathrm{C} 1-\mathrm{C} 7-\mathrm{O} 9)$ & 121.2 & $121.0(3 / 2)$ & $121.3(2 / 1)$ & $121.3(4 / 2)$ & 0.3 \\
$\angle(\mathrm{N} 2-\mathrm{C} 3-\mathrm{C} 4)$ & 121.9 & $122.0(2 / 1)$ & $121.9(1 / 1)$ & $121.9(4 / 2)$ & 0.1 \\
$\angle(\mathrm{C} 3-\mathrm{C} 4-\mathrm{N} 5)$ & 122.5 & $122.4(2 / 1)$ & $122.5(2 / 1)$ & $122.5(4 / 2)$ & 0.1 \\
$\angle(\mathrm{C} 4-\mathrm{N} 5-\mathrm{C} 6)$ & 115.6 & $115.5(3 / 2)$ & $115.5(2 / 1)$ & $115.3(5 / 4)$ & 0.2 \\
$\angle(\mathrm{N} 8-\mathrm{C} 7-\mathrm{O} 9)$ & 125.0 & $125.3(3 / 2)$ & $125.0(2 / 1)$ & $124.5(4 / 3)$ & 0.8 \\
\hline$w R,{ }^{\mathrm{d}} \%$ & & 4.36 & 4.07 & 5.20 & \\
\hline
\end{tabular}

${ }^{a}$ Bond lengths $(\AA)$ and angles (degrees) correspond to equilibrium structure.

${ }^{b}$ In parentheses the first number is the pure experimental standard deviation calculated using method from [13], the second value is the least-squares standard deviation.

${ }^{c}$ Maximal absolute differences between refined values.

d Weighted factor of disagreement between model and experimental $s M(s)$ functions, $w R=\left[\sum w_{i}\left\{s_{i} M\left(s_{i}\right)_{\text {model }}-s_{i} M\left(s_{i}\right)_{\text {exper }}\right\}^{2} / \sum w_{i}\left\{s_{i} M\left(s_{i}\right)_{\text {exper }}\right\}^{2}\right]^{1 / 2} \times 100 \%$

parentheses in Table 1). If both compared sets were refined from experimental data then the weighting factors were calculated as $w_{i}=\left(\sigma_{A,(i)}^{2}+\sigma_{B,(i)}^{2}\right)^{-1}$. When an experimental set $A$ was compared with the theoretical set $\operatorname{CCSD}(\mathrm{T})$ then the weights were calculated as $w_{i}=\sigma_{A,(i)}^{-2}$. All sets of the experimental intensities were used in as much as possible similar way in the structural refinements. Still, their different quality has led to the different values of experimental standard deviations (the definition and a method for their calculation are given in [13]) for the refined parameters. Thus, the usage of these standard deviations in calculations of WRMSD values should lead to a more robust estimation of experimental accuracy and precision. As the values in Table 2 demonstrate, the average discrepancy between experimental 
results is about $0.005 \AA$ for bond lengths and 0.3 degrees for angles. These values express reproducibility of experimental results and are closely related to the precision of the refined parameters. Slightly smaller WRMSD values were obtained comparing experimental and theoretical values. If the structure from ae$\operatorname{CCSD}(\mathrm{T}) /$ cc-pwCVTZ calculation can be taken as a reference, the accuracy of refined parameters is about $0.003 \AA$ for bond lengths and 0.2 degrees for angles. This is in good agreement with results of systematic investigations of Vogts et al., see for example [33-35]. The electronic structure of PZA is relatively simple and can be accurately calculated using single-reference coupled cluster theory, as the calculated value (0.012) of $T_{1}$ diagnostic [36] showed. The situation may, however, change for molecules with larger contribution of static electron correlation. In this case feasible computational methods can be significantly less accurate whereas the accuracy of the GED method expected to be stable for molecules of comparable geometrical complexity.

Table 2: WRMSD for bond lengths ( $\AA$, lower triangle) and angles (degrees, upper triangle) in PZA

\begin{tabular}{ccccc}
\hline & CCSD(T) & UBi & LMSU & ISUCT \\
CCSD(T) & & 0.23 & 0.09 & 0.28 \\
UBi & 0.003 & & 0.17 & 0.37 \\
LMSU & 0.002 & 0.005 & & 0.24 \\
ISUCT & 0.005 & 0.004 & 0.007 & \\
\hline
\end{tabular}

A detailed analysis of the results revealed several parameters with relatively large differences between refined values, see column $\Delta_{\max }$ in Table 1 . The largest difference $0.009 \AA$ was between $\mathrm{C} 1-\mathrm{C} 7$ bond lengths refined from LMSU and ISUCT data. Notable differences were also between sets LMSU and ISUCT for lengths of bonds N2-C3 and N5-C6. This is probably related to imperfections in experimental data. The analysis of standard $\mathrm{CCl}_{4}$ diffraction data from LMSU (Figures S3 and S4 in SI) shows systematic discrepancies at small diffraction angles corresponding to the region about $12.5 \mathrm{~mm}$ away from the center of diffraction patterns. Most likely this was due to a distortion in the shape of the sector device. In the refinement of the molecular structure for PZA this could lead to biased parameters. The problem can be solved by measuring sector function in explicit form and using it for correcting experimental data. For the data from ISUCT this kind of analysis was impossible in the present work since this laboratory routinely measures diffraction patterns of polycrystalline $\mathrm{ZnO}$ for calibration purposes. From these data no particular conclusions can be made regarding unevenness in the sector. The other problem was its large opening in the center, which produces very rapid and large drops in the measured electron diffraction intensity (see Figure S5). An accurate response function may be required in this case. Also, the overall precision of the data from ISUCT set was relatively low as the comparison of Figures 2, 3 and 4 shows. This can be due to the fact that in the standard procedure for data reduction at ISUCT only relatively small area of diffraction pattern is processed. The precision of the LD data from UBi (Figure 2) was also relatively low probably due to suboptimal experimental conditions, which was also confirmed in the analysis of standard $\mathrm{CCl}_{4}$ diffraction patterns (see Figure S1). In general, reproducibility of experimental data can be expressed in terms of experimental $R$-factors [9]. For the data in this work experimental $R$-factors were also calculated and collected in Tables S1-S3. The data from LMSU showed the best values, whereas the data from UBi and especially from ISUCT sets were less precise.

Table 3: WMAD for bond lengths ( $\AA$, lower triangle) and angles (degrees, upper triangle) in PZA

\begin{tabular}{ccccc}
\hline & CCSD(T) & UBi & LMSU & ISUCT \\
CCSD $(\mathrm{T})$ & & 0.18 & 0.06 & 0.21 \\
UBi & 0.003 & & 0.13 & 0.25 \\
LMSU & 0.002 & 0.004 & & 0.16 \\
ISUCT & 0.004 & 0.003 & 0.006 & \\
\hline
\end{tabular}

WRMSD values in Table 2 can be sensitive to pos- 
sible outliers. Therefore we also calculated weighted mean absolute deviations as

$$
\mathrm{WMAD}=\frac{\sum_{i=1}^{N} w_{i}\left|p_{i}^{\mathrm{A}}-p_{i}^{\mathrm{B}}\right|}{\sum_{i=1}^{N} w_{i}}
$$

where all symbols have the same meaning as in equation 1 except for the weighting factors $w_{i}$. The latter were calculated as $w_{i}=\left(\sigma_{A,(i)}^{2}+\sigma_{B,(i)}^{2}\right)^{-1 / 2}$ when both $\mathrm{A}$ and $\mathrm{B}$ were experimental sets and $w_{i}=\sigma_{A,(i)}^{-1}$ when only one of the sets was experimental. The obtained values (see Table 3 ) were only slightly smaller than respective WRMSD.

Table 4: WRMSD ( $\AA$, upper triangle) and WMAD ( $\AA$, lower triangle) for amplitudes of interatomic vibrations in PZA

\begin{tabular}{ccccc}
\hline & B3LYP & UBi & LMSU & ISUCT \\
B3LYP & & 0.007 & 0.002 & 0.002 \\
UBi & 0.006 & & 0.005 & 0.007 \\
LMSU & 0.002 & 0.005 & & 0.002 \\
ISUCT & 0.002 & 0.006 & 0.003 & \\
\hline
\end{tabular}

As the amplitudes of interatomic vibrations were also refined, WRMSD and WMAD values have been calculated for them as well, see Table 4 . The analysis of these values was significantly hindered due to the aforementioned effect of finite sample size. Hence the deviations between refined and theoretical values and between refined values themselves have contributions due to random and systematic errors in measured data and due to differences in experimental setups and conditions. In particular, the latter makes it impossible to require exact agreement between accurately calculated and refined amplitudes if the model does not take into account the effect of finite sample size $[31,32]$ explicitly. However, the results of this work suggest that the setups at LMSU and ISUCT produce data which gives the most accurate amplitudes.

\section{Conclusions and outlook}

Based on the WMAD values the determined average experimental precision of the parameters refined from the GED data is about $0.004 \AA$ for bond lengths and 0.2 degrees for angles. We recommend to take these values into account in calculations of total uncertainties if the complexity of the studied molecule is comparable to that of pyrazinamide. The accuracy of geometrical parameters was also approximately within the stated error limits, although this result has been obtained with a less strong evidence. The average disagreement between refined amplitudes of vibrations was $0.005 \AA$. The present work was limited by using only experimental electron diffraction data and applying flexible restraints in structural refinements. Further investigations are required to determine the true accuracy and precision for parameters refined in substantially different inverse problems.

\section{Acknowledgments}

This work was supported by Deutsche Forschungsgemeinschaft (DFG, Grant VI 713/1-2), by the Ministry of Science and Higher Education of the Russian Federation (Project No. FZZW-2020-0007) and by Russian Foundation for Basic Research (Project No. 20-03-00747 A). YuVV is grateful to HPC facilities at the Universität zu Köln for providing computational time and programs. Many thanks to Norbert W. Mitzel for fruitful discussions and support.

\section{Compliance with ethical stan- dards}

The authors declare no competing financial interest.

\section{$9 \quad$ Keywords}

gas electron diffraction, molecular structure, accuracy, precision, uncertainty, pyrazinamide 


\section{References}

[1] H. Mark and R. Wierl. "The determination of molecular structure by the diffraction of electrons by a stream of vapor." In: Z Elektrochem. Angew. Phys. Chem. 36 (1930), pp. 675-676.

[2] I. Hargittai. "A Survey: The Gas-Phase Electron Diffraction Technique of Molecular Structure Determination". In: Stereochemical Applications of Gas Phase Electron Diffraction, Part A: The Electron Diffraction Technique. Ed. by I. Hargittai and M. Hargittai. VCH Publishers, Inc., New York, 1988.

[3] S. L. Masters, G. V. Girichev, and S. A. Shlykov. "The re-determination of the molecular structure of antimony(III) oxide using very-high-temperature gas electron diffraction (VHT-GED)". In: Dalton Trans. 42.10 (2013), pp. 3581-3586. DOI: 10.1039/c2dt32790b.

[4] A. Almenningen, I. M. Anfinsen, and A. Haaland. "An Electron Diffraction Study of Azomethane, $\mathrm{CH}_{3} \mathrm{NNCH}_{3}$ ". In: Acta Chem. Scand. 24 (1970), pp. 1230-1234. DOI: 10 . 3891/acta.chem. scand.24-1230.

[5] I. Hargittai et al. "Two independent gas electron diffraction investigations of the structure of plumbous chloride". In: J. Mol. Struct. 42 (1977), pp. 147-151. DOI: 10 . $1016 / 0022-$ 2860 (77) 87038-5.

[6] L. Schäfer. "Electron diffraction determinations of gas-phase molecular structures". In: Molecular Structure by Diffraction Methods: Volume 6. Ed. by L. E. Sutton and M. R. Truter. 1978, pp. 1-37. DOI: $10.1039 / 9781847556790-$ 00001.

[7] V. M. Petrov et al. "Three independent studies of the molecular structure of $\mathrm{CdBr}_{2}$ by means of gas-phase electron diffraction". In: J. Struct. Chem. 26.2 (1985), pp. 189-192.

[8] F. Ramondo et al. "Molecular structure of 1,3,5-trifluorobenzene: comparison of the results of two electron diffraction studies". In: $J$. Mol. Struct. 269.3 (1992), pp. 367-373. DOI: 10.1016/0022-2860 (92)85007-4.
[9] Yu. V. Vishnevskii et al. "Molecular Structures of $o$ - and $m$-Fluoro(trifluoromethoxy)benzenes According to Gas Electron Diffraction and Quantum-Chemical Studies: Comparison of the Structures of Trifluoromethoxybenzene and Its Fluorinated Derivatives". In: Russ. J. Phys. Chem. 79.10 (2005), pp. 1537-1547.

[10] A. Campanelli, A. Domenicano, and I. Hargittai. "Comparison of the molecular structures of five benzene derivatives as determined independently by gas-phase electron diffraction in two different laboratories: a perspective". In: Struct. Chem. 21 (2010), pp. 803-808. DOI: 10.1007/ s11224-010-9614-x.

[11] A. Almenningen et al. "The molecular structures of 1,3,5-trichlorobenzene and 1,3,5trifluorobenzene from electron diffraction". In: J. Mol. Struct. 116.1 (1984), pp. 199-206. DOI: 10.1016/0022-2860 (84)80195-7.

[12] R. Blom et al. "The molecular structure of 1,3,5-trichlorobenzene determined by combined analysis of gas-phase electron diffraction and liquid crystal NMR data". In: J. Mol. Struct. 245.3 (1991), pp. 369-377. DOI: 10.1016/00222860 (91) $87111-\mathrm{T}$.

[13] D. S. Tikhonov et al. "Semi-experimental equilibrium structure of pyrazinamide from gasphase electron diffraction. How much experimental is it?" In: J. Mol. Struct. 1132 (2017), pp. 20-27. DOI: $10.1016 / \mathrm{j}$. molstruc . 2016 . 05.090.

[14] R. J. F. Berger et al. "An Improved Gas Electron Diffractometer - The Instrument, Data Collection, Reduction and Structure Refinement Procedures". In: Z. Naturforsch. B 64b.11/12 (2009), pp. 1259-1268.

[15] C. G. Reuter et al. "Gas electron diffraction of increased performance through optimization of nozzle, system design and digital control". In: Z. Naturforsch. B 71.1 (2016), pp. 1-13. DOI: 10.1515/znb-2015-0186. 
[16] Yu. V. Vishnevskiy. "The Initial Processing of the Gas Electron Diffraction Data: an Improved Method for Obtaining Intensity Curves from Diffraction Patterns". In: J. Mol. Struct. 833 (2007), pp. 30-41. DOI: 10.1016/j.molstruc. 2006.08 .026$.

[17] Yu. V. Vishnevskiy. "The Initial Processing of the Gas Electron Diffraction Data: New Method for Simultaneous Determination of the Sector Function and Electron Wavelength from Gas Standard Data". In: J. Mol. Struct. 871.1-3 (2007), pp. 24-32. DOI: 10.1016/j.molstruc. 2007.01 .053$.

[18] Yury V. Vishnevskiy, Sebastian Blomeyer, and Christian G. Reuter. "Gas standards in gas electron diffraction: accurate molecular structures of $\mathrm{CO}_{2}$ and $\mathrm{CCl}_{\text {textrm4 }}$ ". In: Struct. Chem. 31.2 (2020), pp. 667-677. DOI: 10.1007/ s11224-019-01443-5.

[19] G. V. Girichev, A. N. Utkin, and Yu. F. Revichev. "Modernization of the EMR-100 setup for the studies of gases (in Russian)". In: Prib. Tekh. Eksp. 27.2 (1984), pp. 187-190.

[20] G. V. Girichev, S. A. Shlykov, and Yu. F. Revichev. "Apparatus for studies of the molecular structures of non-saturated compounds (in Russian)". In: Prib. Tekh. Eksp. 29.4 (1986), pp. 167-169.

[21] S. A. Shlykov and G. V. Girichev. "A Radiofrequency Mass Spectrometer based on APDM1 unit for the mass range of 1-1600 amu (in Russian)". In: Prib. Tekh. Eksp. 31.2 (1988), pp. 141-142.

[22] E. G. Girichev et al. "Automation of a physicochemical experiment: photometry and voltammetry (in Russian)". In: Izv. Vysh. Uchebn. Zaved., Tekstiln. Prom. 2 (2000), pp. 142-146.

[23] J. F. Stanton et al. CFOUR, Coupled-Cluster techniques for Computational Chemistry, a quantum-chemical program package. With contributions from A.A. Auer, R.J. Bartlett, U. Benedikt, C. Berger, D.E. Bernholdt, Y.J. Bomble, O. Christiansen, F. Engel, R. Faber,
M. Heckert, O. Heun, M. Hilgenberg, C. Huber, T.-C. Jagau, D. Jonsson, J. Jusélius, T. Kirsch, K. Klein, W.J. Lauderdale, F. Lipparini, T. Metzroth, L.A. Mück, D.P. O'Neill, D.R. Price, E. Prochnow, C. Puzzarini, K. Ruud, F. Schiffmann, W. Schwalbach, C. Simmons, S. Stopkowicz, A. Tajti, J. Vázquez, F. Wang, J.D. Watts and the integral packages MOLECULE (J. Almlöf and P.R. Taylor), PROPS (P.R. Taylor), ABACUS (T. Helgaker, H.J. Aa. Jensen, P. Jørgensen, and J. Olsen), and ECP routines by A. V. Mitin and C. van Wüllen. For the current version, see http://www.cfour.de.

[24] Yu. V. Vishnevskiy et al. "Structure and Bonding Nature of the Strained Lewis Acid 3Methyl-1-boraadamantane: A Case Study Employing a New Data-Analysis Procedure in Gas Electron Diffraction". In: Chem. Eur. J. 18.34 (2012), pp. 10585-10594. DOI: 10.1002/chem. 201200264.

[25] Yu. V. Vishnevskiy, 2020, UNEX version 1.6, http://unexprog.org (accessed Mon Jan 20 2020).

[26] A. Borba et al. "Low Temperature Infrared Spectroscopy Study of Pyrazinamide: From the Isolated Monomer to the Stable Low Temperature Crystalline Phase". In: J. Phys. Chem. A 114.1 (2010), pp. 151-161. DOI: 10 .1021/ jp907466h.

[27] T. Baše et al. "Icosahedral Carbaboranes with Peripheral Hydrogen-Chalcogenide Groups: Structures from Gas Electron Diffraction and Chemical Shielding in Solution". In: Chem. Eur. J. 25.9 (2019), pp. 2313-2321. DOI: 10. 1002/chem. 201805145.

[28] M. J. Frisch et al. Gaussian 16 Revision B.01. Gaussian Inc. Wallingford CT. 2016.

[29] Yu. V. Vishnevskiy and Yu. A. Zhabanov. "New implementation of the first-order perturbation theory for calculation of interatomic vibrational amplitudes and corrections in gas electron diffraction". In: J. Phys.: Conf. Ser. 633.1 
(2015), p. 012076. URL: http://stacks.iop. org $/ 1742-6596 / 633 / i=1 / a=012076$.

[30] L. S. Bartell and H. Yow. "Error matrices in gas-electron diffraction I. Effects of systematic errors in intensities". In: J. Mol. Struct. 15.2 (1973), pp. 173-188. DOI: 10 . $1016 / 0022$ 2860 (73) $85001-\mathrm{x}$.

[31] I. L. Karle and J. Karle. "Internal Motion and Molecular Structure Studies by Electron Diffraction. III. Structure of $\mathrm{CH}_{2} \mathrm{CF}_{2}$ and $\mathrm{CF}_{2} \mathrm{CF}_{2}$ ". In: J. Chem. Phys. 18.7 (1950), pp. 963-971. DOI: 10.1063/1.1747820.

[32] R. B. Harvey, F. A. Keidel, and S. H. Bauer. "Some Effects of Nozzle Design on the Diffraction of Electrons by Gases". In: J. Appl. Phys. 21.9 (1950), pp. 860-874. DOI: $10.1063 / 1$. 1699775.

[33] N. Vogt, I. I. Marochkin, and A. N. Rykov. "From the Determination of the Accurate Equilibrium Structure of 1-Methylthymine by Gas Electron Diffraction and Coupled Cluster Computations to the Observation of Methylation and Flexibility Effects in Pyrimidine Nucleobases". In: J. Phys. Chem. A 119.1 (2015), pp. 152-159. DOI: 10.1021/jp508500z.

[34] L. S. Khaikin et al. "The Equilibrium Molecular Structure of 4-Cyanopyridine According to a Combined Analysis of Gas-Phase Electron Diffraction and Microwave Data and CoupledCluster Computations". In: Russ. J. Phys. Chem. A 92.10 (2018), pp. 1970-1974. DOI: 10. $1134 / \mathrm{S} 0036024418100102$.

[35] N. Vogt, I. I. Marochkin, and A. N. Rykov. "Experiment and theory at the convergence limit: accurate equilibrium structure of picolinic acid by gas-phase electron diffraction and coupledcluster computations". In: Phys. Chem. Chem. Phys. 20.15 (2018), pp. 9787-9795. DOI: 10 . 1039/C8CP00310F.

[36] T. J. Lee and P. R. Taylor. "A diagnostic for determining the quality of single-reference electron correlation methods". In: Int. J. Quant. Chem. 36.S23 (1989), pp. 199-207. DOI: 10. 1002/qua. 560360824 . 


\title{
Molecular Structure of Pyrazinamide: a Critical Assessment of Modern Gas Electron Diffraction Data from Three Laboratories Supporting Information
}

\author{
Arseniy A. Otlyotov, Georgiy V. Girichev, Anatolii N. Rykov, Timo Glodde, \\ Yury V. Vishnevskiy
}

Table S1: Conditions of GED experiments at UBi

\begin{tabular}{|c|c|c|}
\hline Parameter/Camera setting & $\mathrm{LD}$ & $\mathrm{MD}$ \\
\hline Nozzle-to-film distance, $\mathrm{mm}$ & 500 & 250 \\
\hline Primary electron beam current, $\mu \mathrm{A}$ & 2.9 & 2.9 \\
\hline Accelerating voltage, $\mathrm{kV}$ & 60 & 60 \\
\hline Nozzle tip temperature, $\mathrm{K}$ & $448(1)$ & $443(1)$ \\
\hline Wavelength of electrons, $\AA$ & $0.04858(2)$ & $0.04873(2)$ \\
\hline Exposure time, s & 10 & $8-10$ \\
\hline Residual gas pressure, ${ }^{\mathrm{a}}$ mbar & $2 \times 10^{-6}$ & $7 \times 10^{-7}$ \\
\hline Recorded plates, substance/standard & $4 / 3$ & $4 / 3$ \\
\hline$s$-range $/ \Delta s, \AA^{-1}$ & $2.6-16.4 / 0.2$ & $6.2-32.0 / 0.2$ \\
\hline Inflection points for background ${ }^{\mathrm{b}}$ & 2 & 3 \\
\hline Experimental weighted $R$-factor, $\%^{\mathrm{c}}$ & 1.52 & 2.97 \\
\hline Experimental $R$-factor, $\% \mathrm{~d}$ & 1.70 & 6.72 \\
\hline
\end{tabular}

${ }^{a}$ During measurements of diffraction patterns for PZA.

${ }^{\mathrm{b}}$ For reduced intensities of PZA.

c Calculated for $s M(s)$ of PZA as

$\left[\sum_{i}^{N} \sum_{j}^{M} w_{j}\left(s_{j} M_{i}\left(s_{j}\right)-s_{j} M_{\mathrm{av}}\left(s_{j}\right)\right)^{2} / N \sum_{j}^{M} w_{j}\left(s_{j} M_{\mathrm{av}}\left(s_{j}\right)\right)^{2}\right]^{1 / 2} \times 100 \%$, where $s_{j} M_{i}\left(s_{j}\right)$ is the

experimental molecular intensity from set $i$ in point $s_{j}, s_{j} M_{\mathrm{av}}\left(s_{j}\right)$ is the average molecular intensity curve in the point $s_{j}, w_{j}$ is the weight of the averaged $s M(s)$ calculated from the respective standard deviation as $1 / \sigma^{2}, \mathrm{~N}$ is the number of data sets, $\mathrm{M}$ is the number of points in each set.

d Calculated with all $w_{j}=1$. 
Table S2: Conditions of GED experiments at LMSU

\begin{tabular}{|c|c|c|}
\hline Parameter/Camera setting & LD & $\mathrm{SD}$ \\
\hline Nozzle-to-film distance, $\mathrm{mm}$ & 362.3 & 193.9 \\
\hline Primary electron beam current, $\mu \mathrm{A}$ & 2.0 & 2.3 \\
\hline Accelerating voltage, $\mathrm{kV}$ & 60 & 60 \\
\hline Nozzle tip temperature, $\mathrm{K}$ & $400(3)$ & $404(2)$ \\
\hline Wavelength of electrons, $\AA$ & $0.04954(7)$ & $0.04996(44)$ \\
\hline Exposure time, s & 30 & 60 \\
\hline Residual gas pressure, ${ }^{\mathrm{a}} \mathrm{mmHg}$ & $3 \times 10^{-5}$ & $3 \times 10^{-5}$ \\
\hline Recorded films, substance/standard & $3 / 2$ & $3 / 2$ \\
\hline$s$-range $/ \Delta s, \AA^{-1}$ & $3.2-19.6 / 0.2$ & $7.2-33.4 / 0.2$ \\
\hline Inflection points for background ${ }^{\mathrm{b}}$ & 2 & 3 \\
\hline Experimental weighted $R$-factor, $\%$ c & 1.22 & 1.73 \\
\hline Experimental $R$-factor, $\%^{\mathrm{c}}$ & 2.22 & 4.54 \\
\hline
\end{tabular}

a During measurements of diffraction patterns for PZA.

b For unmodified intensities of PZA.

c See notes in Table S1.

Table S3: Conditions of GED/MS experiments at ISUCT

\begin{tabular}{lll}
\hline Parameter/Camera setting & LD & $\mathrm{SD}$ \\
Nozzle-to-film distance, mm & 598 & 338 \\
Primary electron beam current, $\mu \mathrm{A}$ & 0.76 & 1.24 \\
Accelerating voltage, $\mathrm{kV}$ & 82 & 83 \\
Temperature of effusion cell, $\mathrm{K}$ & $370(5)$ & $373(5)$ \\
Wavelength of electrons, $\AA$ & $0.04110(3)$ & $0.04081(3)$ \\
Exposure time, s & 96 & 83 \\
Residual gas pressure, Torr & & \\
-in diffraction chamber & $1.4 \times 10^{-6}$ & $1.2 \times 10^{-6}$ \\
-in mass-spectrometric block & $5.6 \times 10^{-7}$ & $6.0 \times 10^{-7}$ \\
Ionization voltage, V & 50 & 50 \\
Recorded films, substance/standard & $5 / 2$ & $5 / 2$ \\
$s$-range/ $\Delta s, \AA^{-1}$ & $1.3-16.2 / 0.1$ & $3.2-28.9 / 0.1$ \\
Inflection points for background & 2 & 3 \\
Experimental weighted $R$-factor, $\%^{\mathrm{b}}$ & 3.92 & 6.64 \\
Experimental $R$-factor, \% & 7.09 & 12.51 \\
\hline
\end{tabular}

${ }^{\text {a }}$ For reduced intensities of PZA.

b See notes in Table S1. 


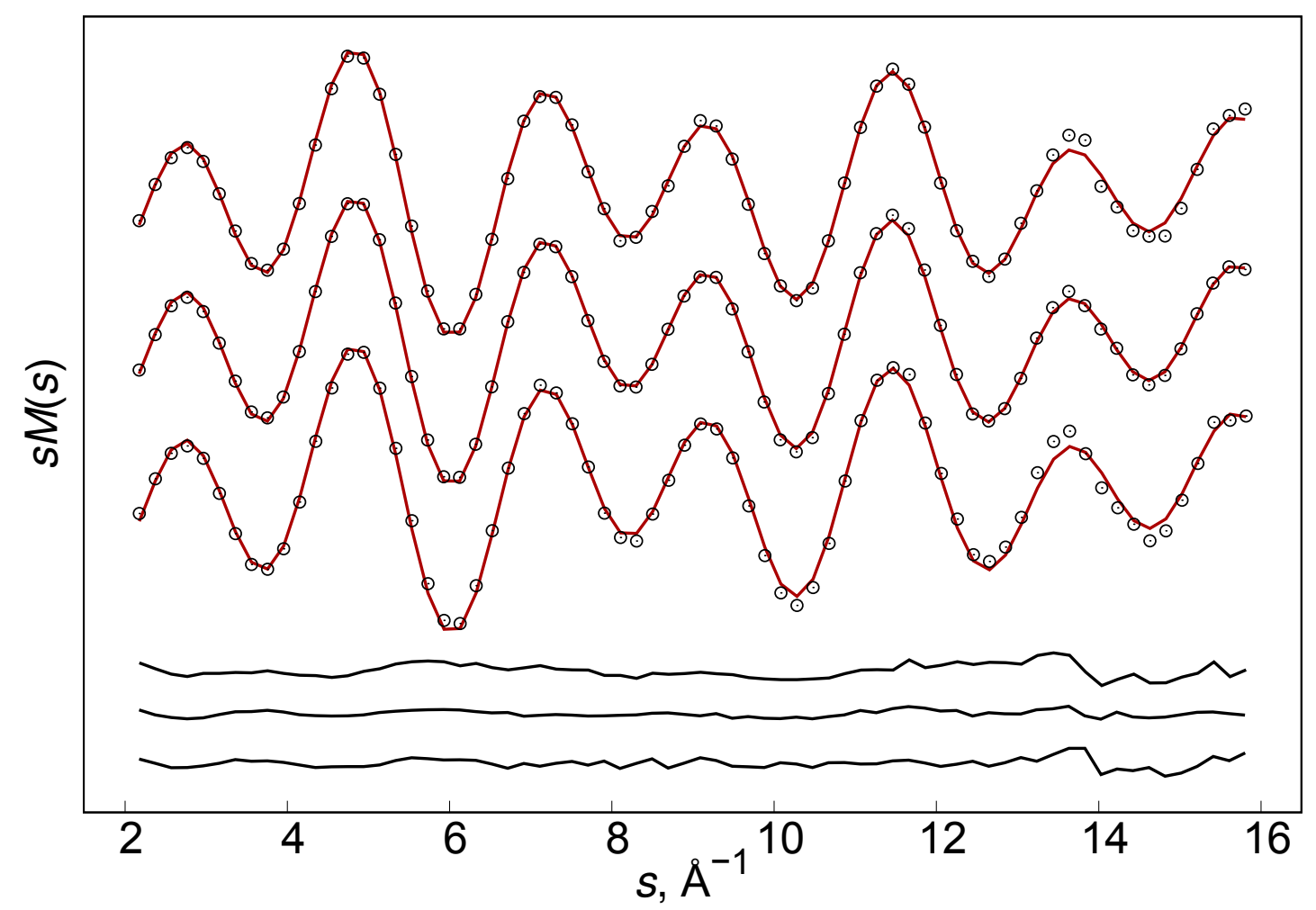

Figure S1: Molecular intensity functions of standard CCl4 measured at UBi (dots), corresponding model (lines) and difference curves. The data for the long nozzle-to-detector camera setting are shown.

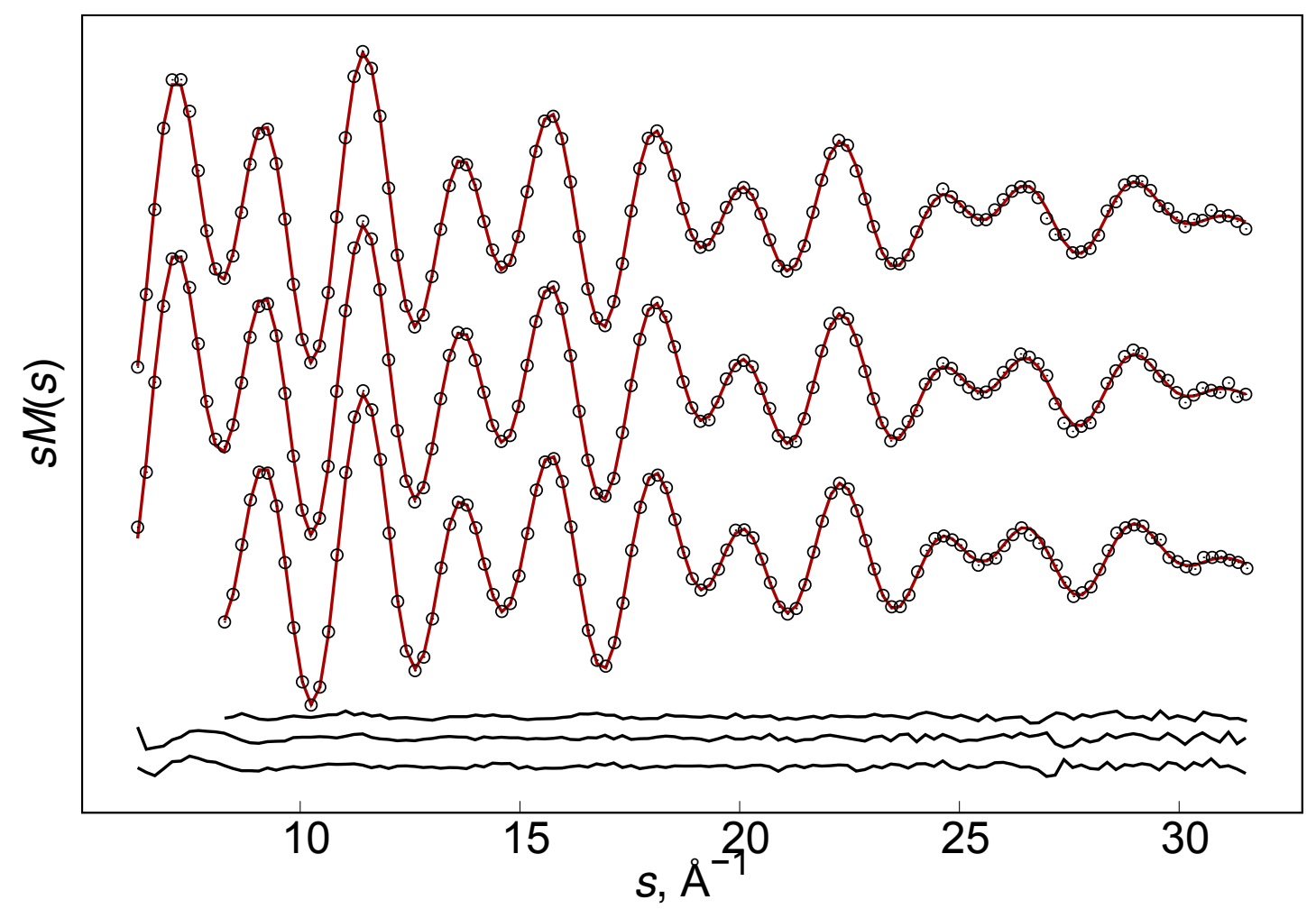

Figure S2: Molecular intensity functions of standard CCl4 measured at UBi (dots), corresponding model (lines) and difference curves. The data for the middle nozzle-to-detector camera setting are shown. 


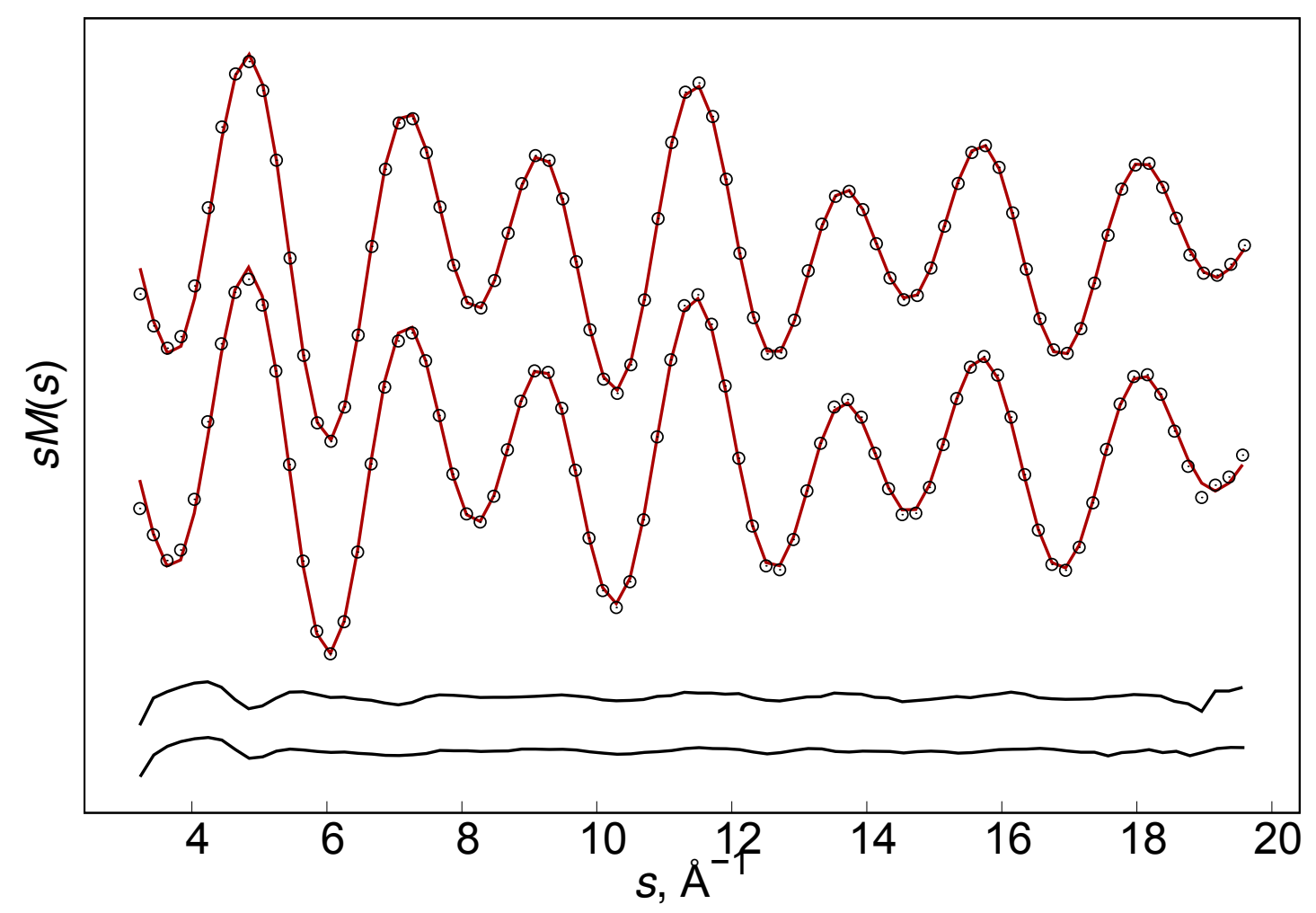

Figure S3: Molecular intensity functions of standard CCl4 measured at LMSU (dots), corresponding model (lines) and difference curves. The data for the long nozzle-to-detector camera setting are shown.

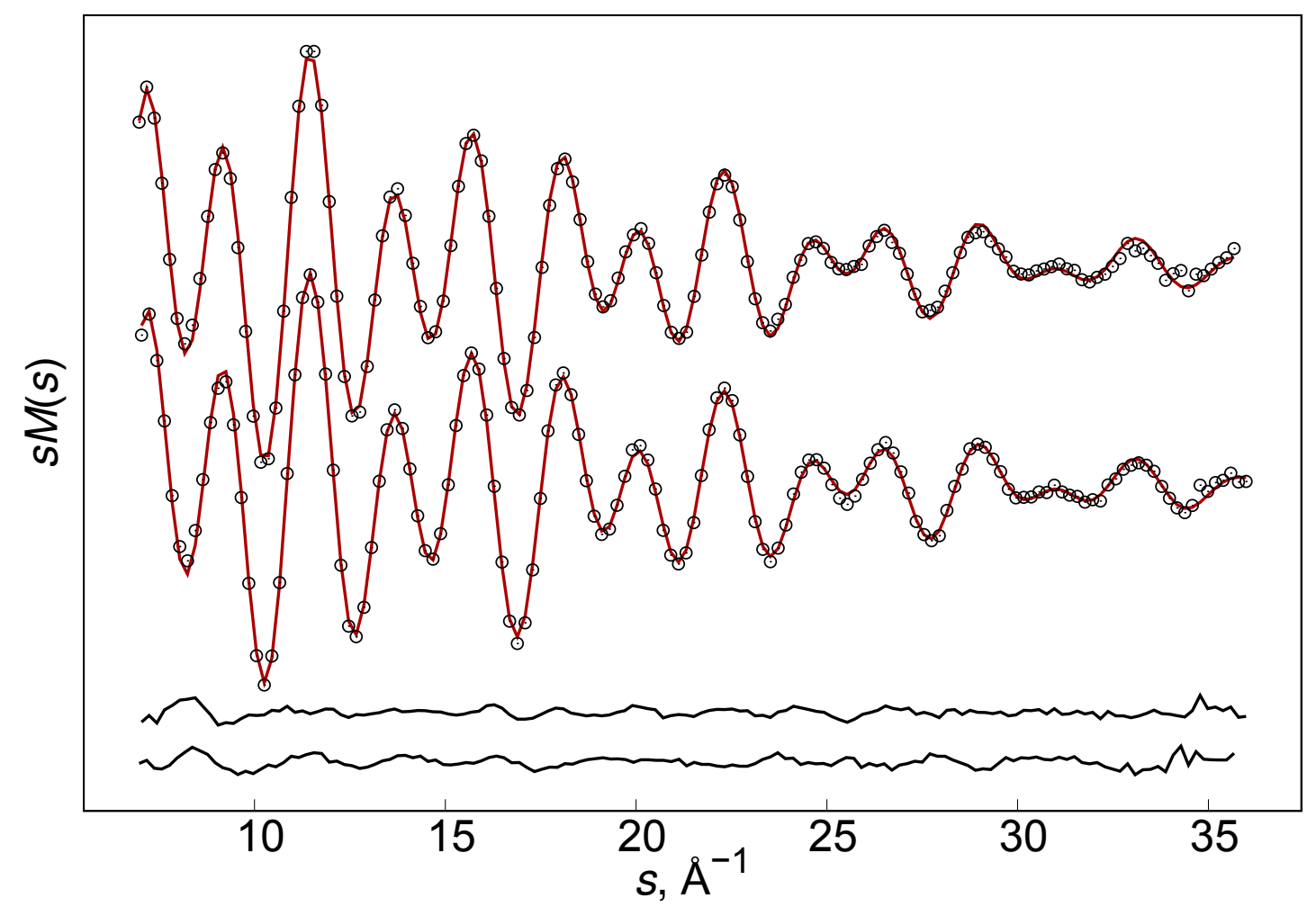

Figure S4: Molecular intensity functions of standard CCl4 measured at LMSU (dots), corresponding model (lines) and difference curves. The data for the short nozzle-to-detector camera setting are shown. 


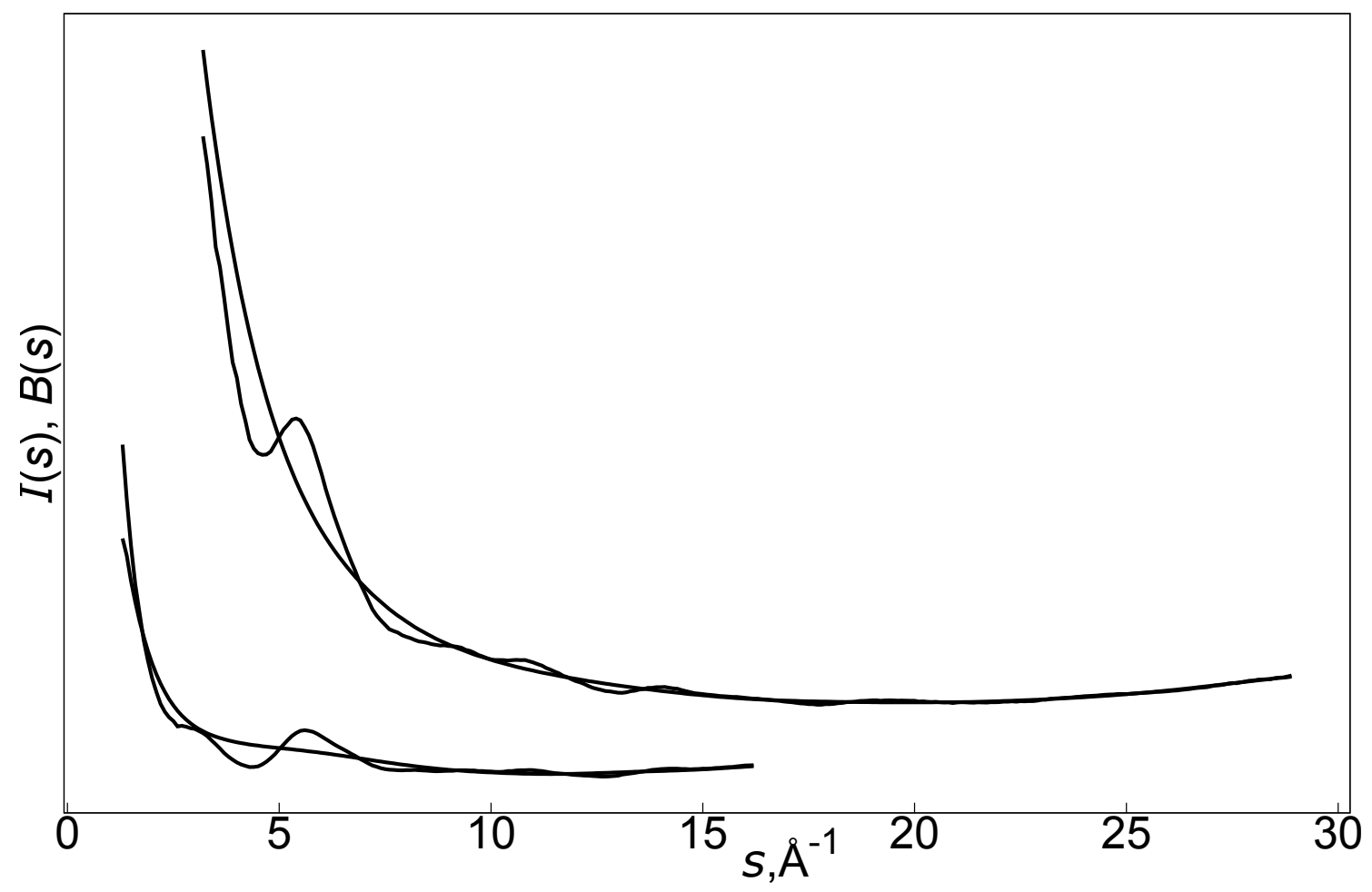

Figure S5: Total intensity functions measured for PZA at ISUCT and corresponding background lines. The data from the first diffraction patterns in LD and SD measurements are shown. 
Table S4: Molecular parameters of PZA by three GED experiments and CCSD(T)/cc-pwCVTZ calculations $^{\mathrm{a}}$

\begin{tabular}{|c|c|c|c|c|}
\hline \multirow{2}{*}{$\begin{array}{c}\text { Method } \\
\text { Parameter }\end{array}$} & \multirow{2}{*}{$\begin{array}{l}\operatorname{CCSD}(\mathrm{T}) / \\
\text { cc-pwCVTZ }\end{array}$} & \multicolumn{3}{|c|}{$\mathrm{GED}^{\mathrm{b}}$} \\
\hline & & UBi & LMSU & ISUCT \\
\hline$r(\mathrm{C} 1-\mathrm{N} 2)$ & 1.337 & $1.333(3 / 2)$ & $1.339(1 / 1)$ & $1.335(4 / 4)$ \\
\hline$r(\mathrm{C} 1-\mathrm{C} 6)$ & 1.394 & $1.398(3 / 2)$ & $1.398(2 / 1)$ & $1.392(4 / 4)$ \\
\hline$r(\mathrm{C} 1-\mathrm{C} 7)$ & 1.505 & $1.501(2 / 2)$ & $1.506(1 / 1)$ & $1.497(3 / 3)$ \\
\hline$r(\mathrm{~N} 2-\mathrm{C} 3)$ & 1.335 & $1.329(3 / 3)$ & $1.337(2 / 1)$ & $1.330(4 / 4)$ \\
\hline$r(\mathrm{C} 3-\mathrm{C} 4)$ & 1.393 & $1.391(3 / 2)$ & $1.397(2 / 1)$ & $1.391(4 / 4)$ \\
\hline$r(\mathrm{C} 3-\mathrm{H} 10)$ & 1.082 & $1.084(11 / 3)$ & $1.082(11 / 2)$ & $1.085(14 / 4)$ \\
\hline$r(\mathrm{C} 4-\mathrm{N} 5)$ & 1.337 & $1.333(3 / 3)$ & $1.338(2 / 1)$ & $1.334(4 / 4)$ \\
\hline$r(\mathrm{C} 4-\mathrm{H} 11)$ & 1.082 & $1.084(11 / 3)$ & $1.083(10 / 2)$ & $1.086(14 / 4)$ \\
\hline$r(\mathrm{~N} 5-\mathrm{C} 6)$ & 1.336 & $1.333(3 / 3)$ & $1.338(2 / 1)$ & $1.330(4 / 4)$ \\
\hline$r(\mathrm{C} 6-\mathrm{H} 12)$ & 1.080 & $1.082(11 / 3)$ & $1.080(10 / 2)$ & $1.084(14 / 4)$ \\
\hline$r(\mathrm{C} 7-\mathrm{N} 8)$ & 1.350 & $1.347(3 / 3)$ & $1.352(2 / 1)$ & $1.348(4 / 4)$ \\
\hline$r(\mathrm{C} 7-\mathrm{O} 9)$ & 1.219 & $1.220(2 / 2)$ & $1.221(1 / 1)$ & $1.214(2 / 2)$ \\
\hline$r(\mathrm{~N} 8-\mathrm{H} 13)$ & 1.003 & $1.006(11 / 3)$ & $1.003(7 / 2)$ & $1.001(14 / 4)$ \\
\hline$r(\mathrm{~N} 8-\mathrm{H} 14)$ & 1.002 & $1.005(10 / 3)$ & $1.002(6 / 2)$ & $1.001(14 / 4)$ \\
\hline$\angle(\mathrm{N} 2-\mathrm{C} 1-\mathrm{C} 6)$ & 122.2 & $121.8(4 / 3)$ & $122.0(2 / 1)$ & $121.7(4 / 4)$ \\
\hline$\angle(\mathrm{N} 2-\mathrm{C} 1-\mathrm{C} 7)$ & 118.5 & $118.3(3 / 2)$ & $118.5(2 / 1)$ & $118.7(4 / 2)$ \\
\hline$\angle(\mathrm{C} 1-\mathrm{N} 2-\mathrm{C} 3)$ & 115.9 & $116.0(2 / 1)$ & $115.9(1 / 1)$ & $116.0(3 / 2)$ \\
\hline$\angle(\mathrm{C} 6-\mathrm{C} 1-\mathrm{C} 7)$ & 119.3 & $119.9(3 / 2)$ & $119.6(2 / 2)$ & $119.5(4 / 4)$ \\
\hline$\angle(\mathrm{C} 1-\mathrm{C} 6-\mathrm{N} 5)$ & 122.0 & $122.2(5 / 3)$ & $122.3(3 / 2)$ & $122.6(8 / 5)$ \\
\hline$\angle(\mathrm{C} 1-\mathrm{C} 6-\mathrm{H} 12)$ & 119.6 & $119.5(8 / 4)$ & $119.4(7 / 2)$ & $119.1(15 / 6)$ \\
\hline$\angle(\mathrm{C} 1-\mathrm{C} 7-\mathrm{N} 8)$ & 113.8 & $113.7(3 / 2)$ & $113.8(2 / 1)$ & $114.2(3 / 2)$ \\
\hline$\angle(\mathrm{C} 1-\mathrm{C} 7-\mathrm{O} 9)$ & 121.2 & $121.0(3 / 2)$ & $121.3(2 / 1)$ & $121.3(4 / 2)$ \\
\hline$\angle(\mathrm{N} 2-\mathrm{C} 3-\mathrm{C} 4)$ & 121.9 & $122.0(2 / 1)$ & $121.9(1 / 1)$ & $121.9(4 / 2)$ \\
\hline$\angle(\mathrm{N} 2-\mathrm{C} 3-\mathrm{H} 10)$ & 117.2 & $117.2(6 / 2)$ & $117.2(4 / 1)$ & $117.2(9 / 3)$ \\
\hline$\angle(\mathrm{C} 4-\mathrm{C} 3-\mathrm{H} 10)$ & 120.9 & $120.8(5 / 2)$ & $120.9(3 / 1)$ & $120.9(8 / 3)$ \\
\hline$\angle(\mathrm{C} 3-\mathrm{C} 4-\mathrm{N} 5)$ & 122.5 & $122.4(2 / 1)$ & $122.5(2 / 1)$ & $122.5(4 / 2)$ \\
\hline$\angle(\mathrm{C} 3-\mathrm{C} 4-\mathrm{H} 11)$ & 120.5 & $120.5(5 / 2)$ & $120.5(4 / 1)$ & $120.5(9 / 3)$ \\
\hline$\angle(\mathrm{N} 5-\mathrm{C} 4-\mathrm{H} 11)$ & 117.0 & $117.1(6 / 2)$ & $117.0(4 / 1)$ & $117.1(8 / 3)$ \\
\hline$\angle(\mathrm{C} 4-\mathrm{N} 5-\mathrm{C} 6)$ & 115.6 & $115.5(3 / 2)$ & $115.5(2 / 1)$ & $115.3(5 / 4)$ \\
\hline$\angle(\mathrm{N} 5-\mathrm{C} 6-\mathrm{H} 12)$ & 118.3 & $118.3(6 / 2)$ & $118.3(5 / 1)$ & $118.4(10 / 3)$ \\
\hline$\angle(\mathrm{N} 8-\mathrm{C} 7-\mathrm{O} 9)$ & 125.0 & $125.3(3 / 2)$ & $125.0(2 / 1)$ & $124.5(4 / 3)$ \\
\hline$\angle(\mathrm{C} 7-\mathrm{N} 8-\mathrm{H} 13)$ & 119.4 & $119.4(28 / 2)$ & $119.4(34 / 1)$ & $119.4(30 / 3)$ \\
\hline$\angle(\mathrm{C} 7-\mathrm{N} 8-\mathrm{H} 14)$ & 119.0 & $119.0(29 / 2)$ & $119.0(24 / 1)$ & $119.1(41 / 3)$ \\
\hline$\angle(\mathrm{H} 13-\mathrm{N} 8-\mathrm{H} 14)$ & 121.6 & $121.6(23 / 2)$ & $121.6(23 / 1)$ & $121.5(28 / 4)$ \\
\hline$w R_{\mathrm{f}},{ }^{\mathrm{c}} \%$ & & 4.36 & 4.07 & 5.20 \\
\hline
\end{tabular}

${ }^{a}$ Calculated and refined from GED data parameters correspond to equilibrium structure.

b Numbers given in parentheses are 1 standard deviations (first is the pure experimental value, second one is from the least-squares analysis).

c Structural $R$-factors. 
Table S5: Refined molecular parameters of PZA from data obtained by averaging total intensities $I(s)$ and molecular intensities $s M(s)^{\mathrm{a}}$

\begin{tabular}{|c|c|c|c|c|c|c|}
\hline \multirow[b]{2}{*}{ Parameter } & \multicolumn{3}{|c|}{ average $s M(s)$} & \multicolumn{3}{|c|}{ average $I(s)$} \\
\hline & $\begin{array}{c}\mathrm{UBi} \\
\alpha=3.0 \cdot 10^{5}\end{array}$ & $\begin{array}{c}\text { LMSU } \\
\alpha=3.0 \cdot 10^{6}\end{array}$ & $\begin{array}{c}\text { ISUCT } \\
\alpha=5.0 \cdot 10^{4}\end{array}$ & $\begin{array}{c}\mathrm{UBi} \\
\alpha=40.0\end{array}$ & $\begin{array}{c}\text { LMSU } \\
\alpha=20.0\end{array}$ & $\begin{array}{c}\text { ISUCT } \\
\alpha=40.0\end{array}$ \\
\hline$r(\mathrm{C} 1$ & $333(3 / 2)$ & $1.339(1 / 1)$ & $1.335(4 / 4)$ & $1.333(3 / 3)$ & $1.337(2 / 2)$ & $1.334(4 / 3)$ \\
\hline$r(\mathrm{C} 1-\mathrm{C} 6)$ & $398(3 / 2)$ & $1.398(2 / 1)$ & $1.392(4 / 4)$ & $1.404(3 / 3)$ & $1.395(2 / 2)$ & $1.393(4 / 4)$ \\
\hline$r(\mathrm{C} 1-\mathrm{C} 7)$ & $1.501(2 / 2)$ & $1.506(1 / 1)$ & $1.497(3 / 3)$ & $1.503(2 / 2)$ & $1.502(2 / 1)$ & $1.498(2 / 2)$ \\
\hline$r(\mathrm{~N} 2-\mathrm{C} 3)$ & $1.329(3 / 3)$ & $1.337(2 / 1)$ & $1.330(4 / 4)$ & $1.328(3 / 3)$ & $1.335(2 / 2)$ & $1.333(4 / 4)$ \\
\hline$r(\mathrm{C} 3-\mathrm{C} 4)$ & $1.391(3 / 2)$ & $1.397(2 / 1)$ & $1.391(4 / 4)$ & $1.394(3 / 3)$ & $1.394(2 / 2)$ & $1.391(4 / 3)$ \\
\hline$r(\mathrm{C} 3-\mathrm{H} 10)$ & $1.084(11 / 3)$ & $1.082(11 / 2)$ & $1.085(14 / 4)$ & $1.085(13 / 4)$ & $1.082(10 / 2)$ & $1.084(12 / 5)$ \\
\hline$r(\mathrm{C} 4-\mathrm{N} 5)$ & $1.333(3 / 3)$ & $1.338(2 / 1)$ & $1.334(4 / 4)$ & $1.331(4 / 3)$ & $1.337(2 / 2)$ & $1.332(4 / 4)$ \\
\hline$r(\mathrm{C} 4-\mathrm{H} 11)$ & $4(11 / 3)$ & $1.083(10 / 2)$ & $1.086(14 / 4)$ & $5(13 / 4)$ & $1.082(10 / 2)$ & $1.084(12 / 5)$ \\
\hline$r(\mathrm{~N} 5-\mathrm{C} 6)$ & $33(3 / 3)$ & $1.338(2 / 1)$ & $1.330(4 / 4)$ & $1.332(4 / 3)$ & $1.337(2 / 2)$ & $1.335(4 / 4)$ \\
\hline$r(\mathrm{C} 6-\mathrm{H} 12)$ & & $1.080(10 / 2)$ & & $1.084(13 / 4)$ & $1.081(10 / 2)$ & $1.082(12 / 5)$ \\
\hline$r(\mathrm{C} 7-\mathrm{N} 8)$ & $347(3 / 3)$ & $1.352(2 / 1)$ & $1.348(4 / 4)$ & $1.344(3 / 3)$ & $1.349(2 / 2)$ & $1.346(4 / 3)$ \\
\hline$r(\mathrm{C} 7-\mathrm{O} 9)$ & $1.220(2 / 2)$ & $1.221(1 / 1)$ & $1.214(2 / 2)$ & $1.216(2 / 1)$ & $1.221(1 / 1)$ & $1.211(2 / 2)$ \\
\hline$r(\mathrm{~N} 8-\mathrm{H} 13)$ & $06(11 / 3)$ & $1.003(7 / 2)$ & /4) & $1 / 4)$ & $1.003(9 / 2)$ & $11 / 4)$ \\
\hline$r(\mathrm{~N} 8-\mathrm{H} 14)$ & $1.005(10 / 3)$ & $1.002(6 / 2)$ & $14 / 4)$ & $1.006(11 / 4)$ & $1.002(9 / 2)$ & $1.000(11 / 4)$ \\
\hline$\angle(\mathrm{N} 2-\mathrm{C} 1-\mathrm{C} 6)$ & & & & & $121.9(3 / 2)$ & $121.8(4 / 3)$ \\
\hline$\angle(\mathrm{N} 2-\mathrm{C} 1-\mathrm{C} 7)$ & $8.3(3 / 2)$ & $118.5(2 / 1)$ & /2) & $8.3(3 / 2)$ & $118.5(2 / 1)$ & $118.8(3 / 2)$ \\
\hline$\angle(\mathrm{C} 1-)$ & & $115.9(1 / 1)$ & & & $116.0(2 / 1)$ & $116.2(2 / 2)$ \\
\hline$\angle(\mathrm{C} 6-\mathrm{C} 1-\mathrm{C} 7)$ & $3 / 2)$ & $119.6(2 / 2)$ & 119 & $/ 3)$ & $119.6(3 / 2)$ & $119.4(3 / 3)$ \\
\hline$\angle(\mathrm{C} 1-\mathrm{C} 6-\mathrm{N} 5)$ & $2.2(5 / 3)$ & $122.3(3 / 2)$ & 122. & $121.3(6 / 4)$ & $122.3(4 / 3)$ & $122.1(6 / 5)$ \\
\hline$\angle(\mathrm{C} 1-\mathrm{C} 6-\mathrm{H} 12)$ & $(8 / 4)$ & $119.4(7 / 2)$ & 119. & $120.4(11 / 5)$ & $119.4(8 / 3)$ & $119.4(10 / 5)$ \\
\hline$\angle(\mathrm{C} 1-\mathrm{C} 7-\mathrm{N} 8)$ & $3.7(3 / 2)$ & $113.8(2 / 1)$ & $114.2(3 / 2)$ & $113.7(3 / 2)$ & $113.8(2 / 1)$ & $114.2(3 / 2)$ \\
\hline & & & & & $121.2(2 / 1)$ & $3 / 2)$ \\
\hline$\angle(\mathrm{N} 2-\mathrm{C} 3-\mathrm{C} 4)$ & $2.0(2 / 1)$ & $121.9(1 / 1)$ & $121.9(4 / 2)$ & $121.9(3 / 2)$ & $121.9(2 / 1)$ & $121.8(3 / 2)$ \\
\hline$\angle(\mathrm{N} 2-\mathrm{C} 3-\mathrm{H} 10)$ & $117.2(6 / 2)$ & $117.2(4 / 1)$ & $117.2(9 / 3)$ & $117.2(8 / 2)$ & $117.2(5 / 1)$ & $117.3(7 / 3)$ \\
\hline$\angle(\mathrm{C} 4-\mathrm{C} 3-\mathrm{H} 10)$ & $0.8(5 / 2)$ & $120.9(3 / 1)$ & 120. & $121.0(7 / 3)$ & $120.9(4 / 2)$ & $120.9(6 / 3)$ \\
\hline$\angle(\mathrm{C} 3-\mathrm{C} 4-\mathrm{N} 5)$ & $122.4(2 / 1)$ & $122.5(2 / 1)$ & $122.5(4 / 2)$ & $122.3(3 / 2)$ & $122.4(2 / 1)$ & $122.3(3 / 2)$ \\
\hline$\angle(\mathrm{C} 3-\mathrm{C} 4-\mathrm{H} 11)$ & $120.5(5 / 2)$ & $120.5(4 / 1)$ & 120. & $120.5(7 / 2)$ & $120.5(5 / 1)$ & $120.4(6 / 3)$ \\
\hline$\angle(\mathrm{N} 5-\mathrm{C} 4-\mathrm{H} 11)$ & $117.1(6 / 2)$ & $117.0(4 / 1)$ & $117.1(8 / 3)$ & $117.2(7 / 3)$ & $117.1(5 / 2)$ & $117.2(6 / 3)$ \\
\hline$\angle(\mathrm{C} 4-\mathrm{N} 5-\mathrm{C} 6)$ & & $115.5(2 / 1)$ & (4) & & $115.5(2 / 2)$ & $115.7(4 / 3)$ \\
\hline$\angle(\mathrm{N} 5-\mathrm{C} 6-\mathrm{H} 12)$ & $118.3(6 / 2)$ & $118.3(5 / 1)$ & $118.4(10 / 3)$ & $118.3(8 / 2)$ & $118.3(5 / 1)$ & $118.4(7 / 3)$ \\
\hline$\angle(\mathrm{N} 8-\mathrm{C} 7-\mathrm{O} 9)$ & $125.3(3 / 2)$ & $125.0(2 / 1)$ & $124.5(4 / 3)$ & $125.2(3 / 3)$ & $125.0(2 / 2)$ & $124.3(3 / 3)$ \\
\hline$\angle(\mathrm{C} 7-\mathrm{N} 8-\mathrm{H} 13)$ & $119.4(28 / 2)$ & $119.4(34 / 1)$ & $119.4(30 / 3)$ & $119.4(38 / 2)$ & $119.4(29 / 1)$ & $119.4(29 / 3)$ \\
\hline$\angle(\mathrm{C} 7-\mathrm{N} 8-\mathrm{H} 14)$ & $119.0(29 / 2)$ & $119.0(24 / 1)$ & $119.1(41 / 3)$ & $119.0(44 / 2)$ & $119.0(32 / 1)$ & $119.0(33 / 3)$ \\
\hline$\angle(\mathrm{H} 13-\mathrm{N} 8-\mathrm{H} 14)$ & $121.6(23 / 2)$ & $121.6(23 / 1)$ & $121.5(28 / 4)$ & $121.6(32 / 3)$ & $121.7(26 / 2)$ & $121.6(24 / 4)$ \\
\hline$w R_{\mathrm{f}},{ }^{\mathrm{c}} \%$ & 4.36 & 4.07 & 5.20 & 4.72 & 4.31 & 5.78 \\
\hline
\end{tabular}

${ }^{a}$ Refined from GED data parameters (bond lengths in $\AA$ and bond angles in degrees) correspond to equilibrium structure.

b Numbers given in parentheses are 1 standard deviations (first is the pure experimental value, second one is from the least-squares analysis).

c Structural $R$-factors. 
Table S6: Contributions of GED data into refined molecular parameters of $\mathrm{PZA}^{\mathrm{a}}$

\begin{tabular}{|c|c|c|c|}
\hline Parameter & $\begin{array}{c}\mathrm{UBi} \\
\alpha=3.0 \cdot 10^{5}\end{array}$ & $\begin{array}{c}\text { LMSU } \\
\alpha=3.0 \cdot 10^{6}\end{array}$ & $\begin{array}{c}\text { ISUCT } \\
\alpha=5.0 \cdot 10^{4}\end{array}$ \\
\hline$r(\mathrm{C} 1-\mathrm{N} 2)$ & 0.8 & 0.8 & 0.8 \\
\hline$r(\mathrm{C} 1-\mathrm{C} 6)$ & 0.9 & 0.6 & 0.8 \\
\hline$r(\mathrm{C} 1-\mathrm{C} 7)$ & 0.9 & 0.8 & 0.9 \\
\hline$r(\mathrm{~N} 2-\mathrm{C} 3)$ & 0.8 & 0.8 & 0.8 \\
\hline$r(\mathrm{C} 3-\mathrm{C} 4)$ & 0.9 & 0.6 & 0.8 \\
\hline$r(\mathrm{C} 3-\mathrm{H} 10)$ & 0.1 & 0.0 & 0.1 \\
\hline$r(\mathrm{C} 4-\mathrm{N} 5)$ & 0.8 & 0.8 & 0.8 \\
\hline$r(\mathrm{C} 4-\mathrm{H} 11)$ & 0.1 & 0.0 & 0.1 \\
\hline$r(\mathrm{~N} 5-\mathrm{C} 6)$ & 0.8 & 0.8 & 0.8 \\
\hline$r(\mathrm{C} 6-\mathrm{H} 12)$ & 0.1 & 0.0 & 0.1 \\
\hline$r(\mathrm{C} 7-\mathrm{N} 8)$ & 0.8 & 0.7 & 0.8 \\
\hline$r(\mathrm{C} 7-\mathrm{O} 9)$ & 0.9 & 0.9 & 0.9 \\
\hline$r(\mathrm{~N} 8-\mathrm{H} 13)$ & 0.1 & 0.1 & 0.1 \\
\hline$r(\mathrm{~N} 8-\mathrm{H} 14)$ & 0.1 & 0.1 & 0.1 \\
\hline$\angle(\mathrm{N} 2-\mathrm{C} 1-\mathrm{C} 6)$ & 0.5 & 0.4 & 0.7 \\
\hline$\angle(\mathrm{N} 2-\mathrm{C} 1-\mathrm{C} 7)$ & 0.4 & 0.2 & 0.4 \\
\hline$\angle(\mathrm{C} 1-\mathrm{N} 2-\mathrm{C} 3)$ & 0.5 & 0.3 & 0.4 \\
\hline$\angle(\mathrm{C} 6-\mathrm{C} 1-\mathrm{C} 7)$ & 0.7 & 0.4 & 0.6 \\
\hline$\angle(\mathrm{C} 1-\mathrm{C} 6-\mathrm{N} 5)$ & 0.6 & 0.4 & 0.5 \\
\hline$\angle(\mathrm{C} 1-\mathrm{C} 6-\mathrm{H} 12)$ & 0.2 & 0.1 & 0.2 \\
\hline$\angle(\mathrm{C} 1-\mathrm{C} 7-\mathrm{N} 8)$ & 0.3 & 0.2 & 0.4 \\
\hline$\angle(\mathrm{C} 1-\mathrm{C} 7-\mathrm{O} 9)$ & 0.4 & 0.2 & 0.3 \\
\hline$\angle(\mathrm{N} 2-\mathrm{C} 3-\mathrm{C} 4)$ & 0.3 & 0.2 & 0.3 \\
\hline$\angle(\mathrm{N} 2-\mathrm{C} 3-\mathrm{H} 10)$ & 0.1 & 0.1 & 0.1 \\
\hline$\angle(\mathrm{C} 4-\mathrm{C} 3-\mathrm{H} 10)$ & 0.2 & 0.1 & 0.1 \\
\hline$\angle(\mathrm{C} 3-\mathrm{C} 4-\mathrm{N} 5)$ & 0.4 & 0.2 & 0.3 \\
\hline$\angle(\mathrm{C} 3-\mathrm{C} 4-\mathrm{H} 11)$ & 0.1 & 0.1 & 0.1 \\
\hline$\angle(\mathrm{N} 5-\mathrm{C} 4-\mathrm{H} 11)$ & 0.2 & 0.1 & 0.2 \\
\hline$\angle(\mathrm{C} 4-\mathrm{N} 5-\mathrm{C} 6)$ & 0.7 & 0.6 & 0.6 \\
\hline$\angle(\mathrm{N} 5-\mathrm{C} 6-\mathrm{H} 12)$ & 0.1 & 0.0 & 0.1 \\
\hline$\angle(\mathrm{N} 8-\mathrm{C} 7-\mathrm{O} 9)$ & 0.6 & 0.5 & 0.7 \\
\hline$\angle(\mathrm{C} 7-\mathrm{N} 8-\mathrm{H} 13)$ & 0.0 & 0.0 & 0.0 \\
\hline$\angle(\mathrm{C} 7-\mathrm{N} 8-\mathrm{H} 14)$ & 0.0 & 0.0 & 0.0 \\
\hline$\angle(\mathrm{H} 13-\mathrm{N} 8-\mathrm{H} 14)$ & 0.0 & 0.0 & 0.0 \\
\hline$l 1$ & 0.4 & 0.2 & 0.3 \\
\hline$l 2$ & 0.9 & 0.8 & 0.8 \\
\hline$l 3$ & 0.3 & 0.1 & 0.3 \\
\hline$l 4$ & 0.9 & 0.7 & 0.8 \\
\hline 15 & 0.6 & 0.4 & 0.6 \\
\hline$l 6$ & 0.1 & 0.0 & 0.1 \\
\hline$l 7$ & 0.4 & 0.2 & 0.3 \\
\hline 18 & 0.3 & 0.2 & 0.3 \\
\hline$l 9$ & 0.2 & 0.1 & 0.2 \\
\hline$l 10$ & 0.0 & 0.0 & 0.0 \\
\hline
\end{tabular}

a The values calculated using the method described in [1]. 
Table S7: Deviations of the refined molecular parameters of PZA ${ }^{a}$ from their starting CCSD(T) values for different values of regularizaton parameter $\alpha$. Molecular intensities $s M(s)$ obtained from UBi GED data were averaged in the refinement procedure.

\begin{tabular}{|c|c|c|c|c|c|c|c|c|c|c|}
\hline \multirow{2}{*}{ Parameter } & \multirow{3}{*}{$\begin{array}{c}\operatorname{CCSD}(\mathrm{T}) \\
r_{\mathrm{e}}\end{array}$} & \multicolumn{9}{|c|}{ GED } \\
\hline & & $\Delta^{\mathrm{b}}$ & $\Delta$ & $\Delta$ & $\Delta$ & $\Delta$ & $\Delta$ & $\Delta$ & $\Delta$ & $\Delta$ \\
\hline$\alpha$ & & $3.0 \mathrm{e} 07$ & $1.0 \mathrm{e} 07$ & $1.0 \mathrm{e} 06$ & $5.0 \mathrm{e} 05$ & $3.0 \mathrm{e} 05$ & $2.0 \mathrm{e} 05$ & $1.0 \mathrm{e} 05$ & $5.0 \mathrm{e} 04$ & $1.0 \mathrm{e} 04$ \\
\hline$r(\mathrm{C} 1-\mathrm{N} 2)$ & 1.337 & -0.001 & -0.001 & -0.004 & -0.004 & -0.004 & -0.004 & -0.004 & -0.003 & 0.002 \\
\hline$r(\mathrm{C} 1-\mathrm{C} 6)$ & 1.394 & 0.000 & -0.001 & 0.000 & 0.002 & 0.003 & 0.005 & 0.008 & 0.012 & 0.024 \\
\hline$r(\mathrm{C} 1-\mathrm{C} 7)$ & 1.505 & 0.000 & -0.001 & -0.003 & -0.003 & -0.004 & -0.004 & -0.004 & -0.005 & -0.009 \\
\hline$r(\mathrm{~N} 2-\mathrm{C} 3)$ & 1.335 & -0.001 & -0.002 & -0.004 & -0.005 & -0.006 & -0.007 & -0.009 & -0.011 & -0.015 \\
\hline$r(\mathrm{C} 3-\mathrm{C} 4)$ & 1.393 & -0.001 & -0.002 & -0.002 & -0.002 & -0.002 & -0.001 & -0.001 & 0.000 & -0.002 \\
\hline$r(\mathrm{C} 3-\mathrm{H} 10)$ & 1.082 & 0.000 & 0.000 & 0.001 & 0.001 & 0.002 & 0.002 & 0.004 & 0.006 & 0.012 \\
\hline$r(\mathrm{C} 4-\mathrm{N} 5)$ & 1.337 & -0.001 & -0.001 & -0.003 & -0.004 & -0.004 & -0.005 & -0.005 & -0.006 & -0.007 \\
\hline$r(\mathrm{C} 4-\mathrm{H} 11)$ & 1.082 & 0.000 & 0.000 & 0.001 & 0.001 & 0.002 & 0.003 & 0.004 & 0.006 & 0.014 \\
\hline$r(\mathrm{~N} 5-\mathrm{C} 6)$ & 1.336 & -0.001 & -0.001 & -0.003 & -0.003 & -0.003 & -0.003 & -0.004 & -0.005 & -0.009 \\
\hline$r(\mathrm{C} 6-\mathrm{H} 12)$ & 1.080 & 0.000 & 0.000 & 0.001 & 0.001 & 0.002 & 0.002 & 0.004 & 0.006 & 0.015 \\
\hline$r(\mathrm{C} 7-\mathrm{N} 8)$ & 1.350 & -0.001 & -0.002 & -0.003 & -0.003 & -0.003 & -0.002 & -0.001 & 0.000 & 0.002 \\
\hline$r(\mathrm{C} 7-\mathrm{O} 9)$ & 1.219 & 0.000 & 0.000 & 0.000 & 0.001 & 0.001 & 0.001 & 0.002 & 0.001 & 0.001 \\
\hline$r(\mathrm{~N} 8-\mathrm{H} 13)$ & 1.003 & 0.000 & 0.000 & 0.001 & 0.001 & 0.002 & 0.003 & 0.007 & 0.012 & 0.028 \\
\hline$r(\mathrm{~N} 8-\mathrm{H} 14)$ & 1.002 & 0.000 & 0.000 & 0.001 & 0.002 & 0.003 & 0.004 & 0.008 & 0.014 & 0.033 \\
\hline$\angle(\mathrm{N} 2-\mathrm{C} 1-\mathrm{C} 6)$ & 122.2 & 0.0 & 0.0 & -0.2 & -0.3 & -0.3 & -0.4 & -0.4 & -0.4 & -0.5 \\
\hline$\angle(\mathrm{N} 2-\mathrm{C} 1-\mathrm{C} 7)$ & 118.5 & 0.0 & 0.0 & -0.1 & -0.2 & -0.2 & -0.3 & -0.3 & -0.2 & 0.3 \\
\hline$\angle(\mathrm{C} 1-\mathrm{N} 2-\mathrm{C} 3)$ & 115.9 & 0.0 & 0.0 & 0.1 & 0.2 & 0.2 & 0.2 & 0.2 & 0.2 & 0.0 \\
\hline$\angle(\mathrm{C} 6-\mathrm{C} 1-\mathrm{C} 7)$ & 119.3 & 0.0 & 0.0 & 0.4 & 0.5 & 0.6 & 0.6 & 0.6 & 0.5 & 0.2 \\
\hline$\angle(\mathrm{C} 1-\mathrm{C} 6-\mathrm{N} 5)$ & 122.0 & 0.0 & 0.0 & 0.2 & 0.2 & 0.1 & 0.1 & -0.1 & -0.3 & -0.8 \\
\hline$\angle(\mathrm{C} 1-\mathrm{C} 6-\mathrm{H} 12)$ & 119.6 & 0.0 & 0.0 & -0.2 & -0.2 & -0.2 & -0.1 & 0.1 & 0.3 & 1.0 \\
\hline$\angle(\mathrm{C} 1-\mathrm{C} 7-\mathrm{N} 8)$ & 113.8 & 0.0 & 0.0 & 0.0 & -0.1 & -0.1 & -0.1 & -0.2 & -0.3 & -0.6 \\
\hline$\angle(\mathrm{C} 1-\mathrm{C} 7-\mathrm{O} 9)$ & 121.2 & 0.0 & 0.0 & -0.1 & -0.2 & -0.2 & -0.3 & -0.4 & -0.6 & -0.8 \\
\hline$\angle(\mathrm{N} 2-\mathrm{C} 3-\mathrm{C} 4)$ & 121.9 & 0.0 & 0.0 & 0.1 & 0.1 & 0.1 & 0.1 & 0.2 & 0.3 & 0.9 \\
\hline$\angle(\mathrm{N} 2-\mathrm{C} 3-\mathrm{H} 10)$ & 117.2 & 0.0 & 0.0 & 0.0 & 0.0 & 0.0 & 0.0 & 0.0 & 0.0 & -0.1 \\
\hline$\angle(\mathrm{C} 4-\mathrm{C} 3-\mathrm{H} 10)$ & 120.9 & 0.0 & 0.0 & -0.1 & -0.1 & -0.1 & -0.2 & -0.2 & -0.3 & -0.7 \\
\hline$\angle(\mathrm{C} 3-\mathrm{C} 4-\mathrm{N} 5)$ & 122.5 & 0.0 & 0.0 & 0.0 & 0.0 & 0.0 & -0.1 & -0.1 & -0.2 & -0.5 \\
\hline$\angle(\mathrm{C} 3-\mathrm{C} 4-\mathrm{H} 11)$ & 120.5 & 0.0 & 0.0 & 0.0 & 0.0 & 0.0 & 0.0 & 0.0 & 0.0 & 0.1 \\
\hline$\angle(\mathrm{N} 5-\mathrm{C} 4-\mathrm{H} 11)$ & 117.0 & 0.0 & 0.0 & 0.0 & 0.0 & 0.1 & 0.1 & 0.1 & 0.2 & 0.4 \\
\hline$\angle(\mathrm{C} 4-\mathrm{N} 5-\mathrm{C} 6)$ & 115.6 & 0.0 & 0.0 & -0.1 & -0.1 & -0.1 & 0.0 & 0.1 & 0.3 & 0.9 \\
\hline$\angle(\mathrm{N} 5-\mathrm{C} 6-\mathrm{H} 12)$ & 118.3 & 0.0 & 0.0 & 0.0 & 0.0 & 0.0 & 0.0 & 0.0 & 0.0 & -0.2 \\
\hline$\angle(\mathrm{N} 8-\mathrm{C} 7-\mathrm{O} 9)$ & 125.0 & 0.0 & 0.0 & 0.1 & 0.2 & 0.3 & 0.4 & 0.7 & 0.9 & 1.4 \\
\hline$\angle(\mathrm{C} 7-\mathrm{N} 8-\mathrm{H} 13)$ & 119.4 & 0.0 & 0.0 & 0.0 & 0.0 & 0.0 & 0.0 & 0.0 & -0.1 & -0.2 \\
\hline$\angle(\mathrm{C} 7-\mathrm{N} 8-\mathrm{H} 14)$ & 119.0 & 0.0 & 0.0 & 0.0 & 0.0 & 0.0 & 0.0 & 0.0 & 0.0 & 0.0 \\
\hline$\angle(\mathrm{H} 13-\mathrm{N} 8-\mathrm{H} 14)$ & 121.6 & 0.0 & 0.0 & 0.0 & 0.0 & 0.0 & 0.0 & 0.0 & 0.0 & 0.2 \\
\hline$w R_{\mathrm{f}},{ }^{\mathrm{c}} \%$ & & 8.80 & 8.32 & 5.09 & 4.65 & 4.36 & 4.15 & 3.88 & 3.46 & 2.67 \\
\hline
\end{tabular}

a Bond lengths and corresponding deviations in $\AA$ and bond angles in degrees.

b Difference between the respective refined and starting (CCSD(T)/cc-pwCVTZ) values.

${ }^{\mathrm{c}}$ Weighted factor of disagreement between model and experimental $s M(s)$ functions. 
Table S8: Deviations of the refined molecular parameters of $\mathrm{PZA}^{\text {a }}$ from their starting CCSD(T) values for different values of regularizaton parameter $\alpha$. Molecular intensities $s M(s)$ obtained from LMSU GED data were averaged in the refinement procedure.

\begin{tabular}{|c|c|c|c|c|c|c|c|c|c|c|}
\hline \multirow{2}{*}{ Parameter } & \multirow{3}{*}{$\begin{array}{c}\operatorname{CCSD}(\mathrm{T}) \\
r_{\mathrm{e}}\end{array}$} & \multicolumn{9}{|c|}{ GED } \\
\hline & & $\Delta^{\mathrm{b}}$ & $\Delta$ & $\Delta$ & $\Delta$ & $\Delta$ & $\Delta$ & $\Delta$ & $\Delta$ & $\Delta$ \\
\hline$\alpha$ & & $3.0 \mathrm{e} 07$ & $1.0 \mathrm{e} 07$ & $3.0 \mathrm{e} 06$ & $1.0 \mathrm{e} 06$ & $5.0 \mathrm{e} 05$ & $3.0 \mathrm{e} 05$ & $1.0 \mathrm{e} 05$ & $5.0 \mathrm{e} 04$ & $1.0 \mathrm{e} 04$ \\
\hline$r(\mathrm{C} 1-\mathrm{N} 2)$ & 1.337 & 0.001 & 0.002 & 0.002 & 0.000 & -0.002 & -0.003 & -0.010 & -0.015 & -0.026 \\
\hline$r(\mathrm{C} 1-\mathrm{C} 6)$ & 1.394 & 0.001 & 0.002 & 0.004 & 0.007 & 0.009 & 0.009 & 0.008 & 0.006 & 0.003 \\
\hline$r(\mathrm{C} 1-\mathrm{C} 7)$ & 1.505 & 0.001 & 0.001 & 0.001 & 0.000 & -0.001 & -0.002 & -0.002 & 0.000 & -0.007 \\
\hline$r(\mathrm{~N} 2-\mathrm{C} 3)$ & 1.335 & 0.001 & 0.002 & 0.002 & 0.002 & 0.002 & 0.004 & 0.009 & 0.013 & 0.015 \\
\hline$r(\mathrm{C} 3-\mathrm{C} 4)$ & 1.393 & 0.001 & 0.002 & 0.004 & 0.007 & 0.009 & 0.011 & 0.016 & 0.019 & 0.034 \\
\hline$r(\mathrm{C} 3-\mathrm{H} 10)$ & 1.082 & 0.000 & 0.000 & 0.000 & 0.000 & 0.000 & 0.000 & 0.000 & -0.001 & -0.003 \\
\hline$r(\mathrm{C} 4-\mathrm{N} 5)$ & 1.337 & 0.001 & 0.001 & 0.002 & 0.001 & 0.000 & 0.000 & -0.001 & 0.000 & 0.000 \\
\hline$r(\mathrm{C} 4-\mathrm{H} 11)$ & 1.082 & 0.000 & 0.000 & 0.000 & 0.001 & 0.002 & 0.003 & 0.005 & 0.007 & 0.019 \\
\hline$r(\mathrm{~N} 5-\mathrm{C} 6)$ & 1.336 & 0.001 & 0.001 & 0.002 & 0.002 & 0.001 & 0.001 & -0.002 & -0.004 & -0.013 \\
\hline$r(\mathrm{C} 6-\mathrm{H} 12)$ & 1.080 & 0.000 & 0.000 & 0.000 & 0.000 & 0.000 & 0.001 & 0.001 & 0.000 & 0.000 \\
\hline$r(\mathrm{C} 7-\mathrm{N} 8)$ & 1.350 & 0.001 & 0.001 & 0.002 & 0.002 & 0.002 & 0.003 & 0.005 & 0.007 & 0.018 \\
\hline$r(\mathrm{C} 7-\mathrm{O} 9)$ & 1.219 & 0.001 & 0.001 & 0.003 & 0.005 & 0.005 & 0.006 & 0.005 & 0.004 & 0.003 \\
\hline$r(\mathrm{~N} 8-\mathrm{H} 13)$ & 1.003 & 0.000 & 0.000 & 0.000 & 0.000 & 0.002 & 0.003 & 0.006 & 0.010 & 0.028 \\
\hline$r(\mathrm{~N} 8-\mathrm{H} 14)$ & 1.002 & 0.000 & 0.000 & 0.000 & -0.001 & 0.000 & 0.000 & -0.001 & -0.002 & 0.000 \\
\hline$\angle(\mathrm{N} 2-\mathrm{C} 1-\mathrm{C} 6)$ & 122.2 & -0.2 & -0.3 & -0.2 & -0.1 & 0.0 & 0.0 & 0.3 & 0.5 & 0.2 \\
\hline$\angle(\mathrm{N} 2-\mathrm{C} 1-\mathrm{C} 7)$ & 118.5 & 0.0 & 0.0 & 0.0 & -0.1 & -0.3 & -0.3 & -0.4 & -0.5 & -0.4 \\
\hline$\angle(\mathrm{C} 1-\mathrm{N} 2-\mathrm{C} 3)$ & 115.9 & 0.0 & 0.0 & 0.1 & 0.1 & 0.2 & 0.2 & 0.2 & 0.2 & 1.0 \\
\hline$\angle(\mathrm{C} 6-\mathrm{C} 1-\mathrm{C} 7)$ & 119.3 & 0.2 & 0.3 & 0.2 & 0.3 & 0.3 & 0.3 & 0.2 & 0.0 & 0.2 \\
\hline$\angle(\mathrm{C} 1-\mathrm{C} 6-\mathrm{N} 5)$ & 122.0 & 0.3 & 0.3 & 0.2 & 0.1 & -0.1 & -0.2 & -0.3 & -0.3 & 0.4 \\
\hline$\angle(\mathrm{C} 1-\mathrm{C} 6-\mathrm{H} 12)$ & 119.6 & -0.3 & -0.3 & -0.2 & -0.1 & 0.0 & 0.1 & 0.1 & 0.0 & -1.3 \\
\hline$\angle(\mathrm{C} 1-\mathrm{C} 7-\mathrm{N} 8)$ & 113.8 & 0.0 & 0.0 & 0.0 & 0.0 & -0.1 & -0.1 & -0.2 & -0.2 & -0.4 \\
\hline$\angle(\mathrm{C} 1-\mathrm{C} 7-\mathrm{O} 9)$ & 121.2 & 0.0 & 0.0 & 0.0 & 0.0 & 0.1 & 0.1 & 0.4 & 0.6 & 1.5 \\
\hline$\angle(\mathrm{N} 2-\mathrm{C} 3-\mathrm{C} 4)$ & 121.9 & 0.0 & 0.0 & 0.0 & 0.0 & -0.1 & -0.1 & -0.3 & -0.4 & -1.3 \\
\hline$\angle(\mathrm{N} 2-\mathrm{C} 3-\mathrm{H} 10)$ & 117.2 & 0.0 & 0.0 & 0.0 & 0.0 & 0.0 & 0.0 & -0.1 & -0.2 & -0.7 \\
\hline$\angle(\mathrm{C} 4-\mathrm{C} 3-\mathrm{H} 10)$ & 120.9 & 0.0 & 0.0 & 0.0 & 0.0 & 0.1 & 0.1 & 0.4 & 0.6 & 2.0 \\
\hline$\angle(\mathrm{C} 3-\mathrm{C} 4-\mathrm{N} 5)$ & 122.5 & 0.0 & 0.0 & 0.0 & -0.1 & -0.2 & -0.3 & -0.6 & -0.9 & -1.0 \\
\hline$\angle(\mathrm{C} 3-\mathrm{C} 4-\mathrm{H} 11)$ & 120.5 & 0.0 & 0.0 & 0.0 & 0.0 & 0.0 & 0.0 & 0.0 & 0.0 & 0.3 \\
\hline$\angle(\mathrm{N} 5-\mathrm{C} 4-\mathrm{H} 11)$ & 117.0 & 0.0 & 0.0 & 0.0 & 0.1 & 0.2 & 0.3 & 0.6 & 0.8 & 0.7 \\
\hline$\angle(\mathrm{C} 4-\mathrm{N} 5-\mathrm{C} 6)$ & 115.6 & -0.2 & -0.2 & -0.1 & 0.0 & 0.2 & 0.4 & 0.7 & 0.9 & 0.8 \\
\hline$\angle(\mathrm{N} 5-\mathrm{C} 6-\mathrm{H} 12)$ & 118.3 & 0.0 & 0.0 & 0.0 & 0.0 & 0.1 & 0.1 & 0.2 & 0.3 & 0.8 \\
\hline$\angle(\mathrm{N} 8-\mathrm{C} 7-\mathrm{O} 9)$ & 125.0 & 0.0 & 0.0 & 0.0 & 0.0 & 0.1 & 0.0 & -0.1 & -0.3 & -1.1 \\
\hline$\angle(\mathrm{C} 7-\mathrm{N} 8-\mathrm{H} 13)$ & 119.4 & 0.0 & 0.0 & 0.0 & 0.0 & 0.0 & 0.0 & 0.0 & 0.0 & -0.3 \\
\hline$\angle(\mathrm{C} 7-\mathrm{N} 8-\mathrm{H} 14)$ & 119.0 & 0.0 & 0.0 & 0.0 & 0.0 & -0.1 & -0.1 & -0.2 & -0.4 & -1.2 \\
\hline$\angle(\mathrm{H} 13-\mathrm{N} 8-\mathrm{H} 14)$ & 121.6 & 0.0 & 0.0 & 0.0 & 0.0 & 0.1 & 0.1 & 0.2 & 0.4 & 1.5 \\
\hline$w R_{\mathrm{f}},{ }^{\mathrm{c}} \%$ & & 4.67 & 4.43 & 4.07 & 3.72 & 3.81 & 3.65 & 3.34 & 3.15 & 2.84 \\
\hline
\end{tabular}

a Bond lengths and corresponding deviations in $\AA$ and bond angles in degrees.

b Difference between the respective refined and starting (CCSD(T)/cc-pwCVTZ) values.

${ }^{\mathrm{c}}$ Weighted factor of disagreement between model and experimental $s M(s)$ functions. 
Table S9: Deviations of the refined molecular parameters of $\mathrm{PZA}^{\text {a }}$ from their starting CCSD(T) values for different values of regularizaton parameter $\alpha$. Molecular intensities $s M(s)$ obtained from ISUCT GED data were averaged in the refinement procedure.

\begin{tabular}{|c|c|c|c|c|c|c|c|c|c|c|}
\hline \multirow{2}{*}{ Parameter } & \multirow{3}{*}{$\begin{array}{c}\operatorname{CCSD}(\mathrm{T}) \\
r_{\mathrm{e}}\end{array}$} & \multicolumn{9}{|c|}{ GED } \\
\hline & & $\Delta^{\mathrm{b}}$ & $\Delta$ & $\Delta$ & $\Delta$ & $\Delta$ & $\Delta$ & $\Delta$ & $\Delta$ & $\Delta$ \\
\hline$\alpha$ & & $3.0 \mathrm{e} 07$ & $1.0 \mathrm{e} 07$ & $1.0 \mathrm{e} 06$ & $5.0 \mathrm{e} 05$ & $3.0 \mathrm{e} 05$ & $2.0 \mathrm{e} 05$ & $1.0 \mathrm{e} 05$ & $5.0 \mathrm{e} 04$ & $1.0 \mathrm{e} 04$ \\
\hline$r(\mathrm{C} 1-\mathrm{N} 2)$ & 1.337 & 0.000 & 0.000 & -0.002 & -0.002 & -0.003 & -0.003 & -0.003 & -0.002 & 0.004 \\
\hline$r(\mathrm{C} 1-\mathrm{C} 6)$ & 1.394 & 0.000 & 0.000 & -0.001 & -0.002 & -0.002 & -0.003 & -0.003 & -0.002 & 0.002 \\
\hline$r(\mathrm{C} 1-\mathrm{C} 7)$ & 1.505 & 0.000 & 0.000 & -0.002 & -0.003 & -0.004 & -0.005 & -0.006 & -0.008 & -0.012 \\
\hline$r(\mathrm{~N} 2-\mathrm{C} 3)$ & 1.335 & 0.000 & 0.000 & -0.002 & -0.002 & -0.003 & -0.003 & -0.003 & -0.004 & -0.016 \\
\hline$r(\mathrm{C} 3-\mathrm{C} 4)$ & 1.393 & 0.000 & 0.000 & -0.001 & -0.002 & -0.002 & -0.002 & -0.002 & -0.002 & -0.002 \\
\hline$r(\mathrm{C} 3-\mathrm{H} 10)$ & 1.082 & 0.000 & 0.000 & 0.000 & 0.001 & 0.001 & 0.001 & 0.002 & 0.003 & 0.010 \\
\hline$r(\mathrm{C} 4-\mathrm{N} 5)$ & 1.337 & 0.000 & 0.000 & -0.001 & -0.002 & -0.002 & -0.002 & -0.002 & -0.002 & -0.001 \\
\hline$r(\mathrm{C} 4-\mathrm{H} 11)$ & 1.082 & 0.000 & 0.000 & 0.000 & 0.001 & 0.001 & 0.001 & 0.002 & 0.004 & 0.012 \\
\hline$r(\mathrm{~N} 5-\mathrm{C} 6)$ & 1.336 & 0.000 & 0.000 & -0.002 & -0.002 & -0.003 & -0.003 & -0.004 & -0.006 & -0.013 \\
\hline$r(\mathrm{C} 6-\mathrm{H} 12)$ & 1.080 & 0.000 & 0.000 & 0.000 & 0.001 & 0.001 & 0.001 & 0.002 & 0.003 & 0.008 \\
\hline$r(\mathrm{C} 7-\mathrm{N} 8)$ & 1.350 & 0.000 & 0.000 & -0.002 & -0.002 & -0.003 & -0.003 & -0.003 & -0.002 & 0.007 \\
\hline$r(\mathrm{C} 7-\mathrm{O} 9)$ & 1.219 & 0.000 & 0.000 & -0.001 & -0.002 & -0.002 & -0.003 & -0.004 & -0.005 & -0.007 \\
\hline$r(\mathrm{~N} 8-\mathrm{H} 13)$ & 1.003 & 0.000 & 0.000 & 0.000 & 0.000 & -0.001 & -0.001 & -0.001 & -0.003 & -0.010 \\
\hline$r(\mathrm{~N} 8-\mathrm{H} 14)$ & 1.002 & 0.000 & 0.000 & 0.000 & 0.000 & 0.000 & 0.000 & -0.001 & -0.001 & -0.005 \\
\hline$\angle(\mathrm{N} 2-\mathrm{C} 1-\mathrm{C} 6)$ & 122.2 & 0.0 & 0.0 & 0.1 & 0.0 & -0.1 & -0.1 & -0.3 & -0.4 & -0.5 \\
\hline$\angle(\mathrm{N} 2-\mathrm{C} 1-\mathrm{C} 7)$ & 118.5 & 0.0 & 0.0 & 0.0 & 0.0 & 0.0 & 0.0 & 0.1 & 0.2 & 0.5 \\
\hline$\angle(\mathrm{C} 1-\mathrm{N} 2-\mathrm{C} 3)$ & 115.9 & 0.0 & 0.0 & 0.0 & 0.0 & 0.0 & 0.0 & 0.1 & 0.1 & 0.5 \\
\hline$\angle(\mathrm{C} 6-\mathrm{C} 1-\mathrm{C} 7)$ & 119.3 & 0.0 & 0.0 & -0.1 & 0.0 & 0.0 & 0.1 & 0.1 & 0.2 & 0.0 \\
\hline$\angle(\mathrm{C} 1-\mathrm{C} 6-\mathrm{N} 5)$ & 122.0 & 0.0 & 0.0 & -0.1 & 0.0 & 0.1 & 0.2 & 0.4 & 0.5 & 0.0 \\
\hline$\angle(\mathrm{C} 1-\mathrm{C} 6-\mathrm{H} 12)$ & 119.6 & 0.0 & 0.0 & 0.1 & 0.0 & -0.2 & -0.3 & -0.4 & -0.6 & -0.3 \\
\hline$\angle(\mathrm{C} 1-\mathrm{C} 7-\mathrm{N} 8)$ & 113.8 & 0.0 & 0.0 & 0.0 & 0.1 & 0.1 & 0.2 & 0.3 & 0.4 & 1.1 \\
\hline$\angle(\mathrm{C} 1-\mathrm{C} 7-\mathrm{O} 9)$ & 121.2 & 0.0 & 0.0 & 0.0 & 0.0 & 0.0 & 0.0 & 0.1 & 0.1 & 0.1 \\
\hline$\angle(\mathrm{N} 2-\mathrm{C} 3-\mathrm{C} 4)$ & 121.9 & 0.0 & 0.0 & 0.0 & 0.0 & 0.0 & 0.0 & 0.0 & 0.0 & -0.2 \\
\hline$\angle(\mathrm{N} 2-\mathrm{C} 3-\mathrm{H} 10)$ & 117.2 & 0.0 & 0.0 & 0.0 & 0.0 & 0.0 & 0.0 & 0.0 & 0.0 & 0.2 \\
\hline$\angle(\mathrm{C} 4-\mathrm{C} 3-\mathrm{H} 10)$ & 120.9 & 0.0 & 0.0 & 0.0 & 0.0 & 0.0 & 0.0 & -0.1 & -0.1 & 0.0 \\
\hline$\angle(\mathrm{C} 3-\mathrm{C} 4-\mathrm{N} 5)$ & 122.5 & 0.0 & 0.0 & 0.0 & 0.0 & 0.0 & 0.0 & 0.0 & 0.0 & 0.1 \\
\hline$\angle(\mathrm{C} 3-\mathrm{C} 4-\mathrm{H} 11)$ & 120.5 & 0.0 & 0.0 & 0.0 & 0.0 & 0.0 & 0.0 & 0.0 & 0.0 & -0.2 \\
\hline$\angle(\mathrm{N} 5-\mathrm{C} 4-\mathrm{H} 11)$ & 117.0 & 0.0 & 0.0 & 0.0 & 0.0 & 0.0 & 0.0 & 0.0 & 0.0 & 0.1 \\
\hline$\angle(\mathrm{C} 4-\mathrm{N} 5-\mathrm{C} 6)$ & 115.6 & 0.0 & 0.0 & 0.0 & 0.0 & -0.1 & -0.2 & -0.2 & -0.3 & 0.2 \\
\hline$\angle(\mathrm{N} 5-\mathrm{C} 6-\mathrm{H} 12)$ & 118.3 & 0.0 & 0.0 & 0.0 & 0.0 & 0.0 & 0.0 & 0.0 & 0.1 & 0.3 \\
\hline$\angle(\mathrm{N} 8-\mathrm{C} 7-\mathrm{O} 9)$ & 125.0 & 0.0 & 0.0 & 0.0 & -0.1 & -0.1 & -0.2 & -0.3 & -0.5 & -1.2 \\
\hline$\angle(\mathrm{C} 7-\mathrm{N} 8-\mathrm{H} 13)$ & 119.4 & 0.0 & 0.0 & 0.0 & 0.0 & 0.0 & 0.0 & 0.0 & 0.1 & 0.4 \\
\hline$\angle(\mathrm{C} 7-\mathrm{N} 8-\mathrm{H} 14)$ & 119.0 & 0.0 & 0.0 & 0.0 & 0.0 & 0.0 & 0.0 & 0.0 & 0.1 & 0.3 \\
\hline$\angle(\mathrm{H} 13-\mathrm{N} 8-\mathrm{H} 14)$ & 121.6 & 0.0 & 0.0 & 0.0 & 0.0 & 0.0 & 0.0 & -0.1 & -0.1 & -0.7 \\
\hline$w R_{\mathrm{f}},{ }^{\mathrm{c}} \%$ & & 6.38 & 6.32 & 5.99 & 5.73 & 5.64 & 5.36 & 5.30 & 5.20 & 4.89 \\
\hline
\end{tabular}

a Bond lengths and corresponding deviations in $\AA$ and bond angles in degrees.

b Difference between the respective refined and starting (CCSD(T)/cc-pwCVTZ) values.

${ }^{c}$ Weighted factor of disagreement between model and experimental $s M(s)$ functions. 
Table S10: Deviations of the refined molecular parameters of $\mathrm{PZA}^{\mathrm{a}}$ from their starting $\mathrm{CCSD}(\mathrm{T})$ values for different values of regularizaton parameter $\alpha$. Total intensities $I(s)$ obtained from UBi GED data were averaged in the refinement procedure.

\begin{tabular}{|c|c|c|c|c|c|c|c|c|c|}
\hline \multirow{2}{*}{ Parameter } & \multirow{2}{*}{$\operatorname{CCSD}(\mathrm{T})$} & \multicolumn{8}{|c|}{ GED } \\
\hline & & $\Delta^{\mathrm{b}}$ & $\Delta$ & $\Delta$ & $\Delta$ & $\Delta$ & $\Delta$ & $\Delta$ & $\Delta$ \\
\hline$\alpha$ & & 200.0 & 150.0 & 100.0 & 80.0 & 60.0 & 40.0 & 20.0 & 1.0 \\
\hline$r(\mathrm{C} 1-\mathrm{N} 2)$ & 1.337 & -0.003 & -0.003 & -0.004 & -0.004 & -0.004 & -0.004 & -0.004 & 0.003 \\
\hline$r(\mathrm{C} 1-\mathrm{C} 6)$ & 1.394 & 0.001 & 0.001 & 0.003 & 0.004 & 0.006 & 0.010 & 0.016 & 0.039 \\
\hline$r(\mathrm{C} 1-\mathrm{C} 7)$ & 1.505 & -0.001 & -0.001 & -0.001 & -0.001 & -0.002 & -0.002 & -0.003 & -0.012 \\
\hline$r(\mathrm{~N} 2-\mathrm{C} 3)$ & 1.335 & -0.004 & -0.004 & -0.005 & -0.005 & -0.006 & -0.007 & -0.008 & -0.007 \\
\hline$r(\mathrm{C} 3-\mathrm{C} 4)$ & 1.393 & -0.001 & -0.001 & -0.001 & 0.000 & 0.001 & 0.002 & 0.003 & -0.011 \\
\hline$r(\mathrm{C} 3-\mathrm{H} 10)$ & 1.082 & 0.001 & 0.001 & 0.002 & 0.002 & 0.002 & 0.003 & 0.005 & 0.027 \\
\hline$r(\mathrm{C} 4-\mathrm{N} 5)$ & 1.337 & -0.004 & -0.004 & -0.004 & -0.005 & -0.005 & -0.005 & -0.006 & -0.007 \\
\hline$r(\mathrm{C} 4-\mathrm{H} 11)$ & 1.082 & 0.001 & 0.001 & 0.002 & 0.002 & 0.002 & 0.003 & 0.005 & 0.023 \\
\hline$r(\mathrm{~N} 5-\mathrm{C} 6)$ & 1.336 & -0.003 & -0.003 & -0.004 & -0.004 & -0.004 & -0.004 & -0.005 & -0.012 \\
\hline$r(\mathrm{C} 6-\mathrm{H} 12)$ & 1.080 & 0.001 & 0.001 & 0.002 & 0.002 & 0.002 & 0.003 & 0.005 & 0.027 \\
\hline$r(\mathrm{C} 7-\mathrm{N} 8)$ & 1.350 & -0.004 & -0.004 & -0.005 & -0.005 & -0.005 & -0.006 & -0.007 & 0.001 \\
\hline$r(\mathrm{C} 7-\mathrm{O} 9)$ & 1.219 & -0.001 & -0.001 & -0.001 & -0.001 & -0.002 & -0.002 & -0.004 & 0.001 \\
\hline$r(\mathrm{~N} 8-\mathrm{H} 13)$ & 1.003 & 0.001 & 0.001 & 0.001 & 0.002 & 0.002 & 0.003 & 0.005 & 0.033 \\
\hline$r(\mathrm{~N} 8-\mathrm{H} 14)$ & 1.002 & 0.001 & 0.001 & 0.002 & 0.002 & 0.003 & 0.004 & 0.007 & 0.041 \\
\hline$\angle(\mathrm{N} 2-\mathrm{C} 1-\mathrm{C} 6)$ & 122.2 & -0.2 & -0.2 & -0.1 & -0.1 & 0.0 & 0.1 & 0.2 & -0.1 \\
\hline$\angle(\mathrm{N} 2-\mathrm{C} 1-\mathrm{C} 7)$ & 118.5 & -0.1 & -0.1 & -0.1 & -0.2 & -0.2 & -0.2 & -0.2 & 0.6 \\
\hline$\angle(\mathrm{C} 1-\mathrm{N} 2-\mathrm{C} 3)$ & 115.9 & 0.1 & 0.1 & 0.1 & 0.1 & 0.2 & 0.2 & 0.2 & -0.5 \\
\hline$\angle(\mathrm{C} 6-\mathrm{C} 1-\mathrm{C} 7)$ & 119.3 & 0.3 & 0.3 & 0.3 & 0.2 & 0.2 & 0.1 & 0.0 & -0.6 \\
\hline$\angle(\mathrm{C} 1-\mathrm{C} 6-\mathrm{N} 5)$ & 122.0 & 0.1 & 0.0 & -0.2 & -0.3 & -0.5 & -0.8 & -1.2 & -1.9 \\
\hline$\angle(\mathrm{C} 1-\mathrm{C} 6-\mathrm{H} 12)$ & 119.6 & -0.1 & 0.0 & 0.2 & 0.3 & 0.5 & 0.8 & 1.2 & 2.1 \\
\hline$\angle(\mathrm{C} 1-\mathrm{C} 7-\mathrm{N} 8)$ & 113.8 & 0.0 & 0.0 & 0.0 & 0.0 & 0.0 & -0.1 & -0.2 & -0.5 \\
\hline$\angle(\mathrm{C} 1-\mathrm{C} 7-\mathrm{O} 9)$ & 121.2 & 0.0 & 0.0 & -0.1 & -0.1 & -0.1 & -0.1 & -0.2 & -0.1 \\
\hline$\angle(\mathrm{N} 2-\mathrm{C} 3-\mathrm{C} 4)$ & 121.9 & 0.0 & 0.0 & 0.0 & 0.0 & 0.0 & 0.0 & 0.0 & 1.2 \\
\hline$\angle(\mathrm{N} 2-\mathrm{C} 3-\mathrm{H} 10)$ & 117.2 & 0.0 & 0.0 & 0.0 & 0.0 & 0.0 & 0.0 & 0.0 & 0.0 \\
\hline$\angle(\mathrm{C} 4-\mathrm{C} 3-\mathrm{H} 10)$ & 120.9 & 0.0 & 0.0 & 0.0 & 0.0 & 0.0 & 0.0 & 0.0 & -1.2 \\
\hline$\angle(\mathrm{C} 3-\mathrm{C} 4-\mathrm{N} 5)$ & 122.5 & 0.0 & 0.0 & -0.1 & -0.1 & -0.1 & -0.1 & -0.2 & -0.5 \\
\hline$\angle(\mathrm{C} 3-\mathrm{C} 4-\mathrm{H} 11)$ & 120.5 & 0.0 & 0.0 & 0.0 & 0.0 & 0.0 & 0.0 & 0.0 & 0.1 \\
\hline$\angle(\mathrm{N} 5-\mathrm{C} 4-\mathrm{H} 11)$ & 117.0 & 0.0 & 0.0 & 0.1 & 0.1 & 0.1 & 0.1 & 0.2 & 0.4 \\
\hline$\angle(\mathrm{C} 4-\mathrm{N} 5-\mathrm{C} 6)$ & 115.6 & 0.0 & 0.1 & 0.2 & 0.3 & 0.4 & 0.6 & 1.0 & 1.8 \\
\hline$\angle(\mathrm{N} 5-\mathrm{C} 6-\mathrm{H} 12)$ & 118.3 & 0.0 & 0.0 & 0.0 & 0.0 & 0.0 & 0.0 & 0.0 & -0.2 \\
\hline$\angle(\mathrm{N} 8-\mathrm{C} 7-\mathrm{O} 9)$ & 125.0 & 0.0 & 0.0 & 0.1 & 0.1 & 0.1 & 0.2 & 0.3 & 0.6 \\
\hline$\angle(\mathrm{C} 7-\mathrm{N} 8-\mathrm{H} 13)$ & 119.4 & 0.0 & 0.0 & 0.0 & 0.0 & 0.0 & 0.0 & 0.0 & 0.3 \\
\hline$\angle(\mathrm{C} 7-\mathrm{N} 8-\mathrm{H} 14)$ & 119.0 & 0.0 & 0.0 & 0.0 & 0.0 & 0.0 & 0.0 & 0.0 & 0.2 \\
\hline$\angle(\mathrm{H} 13-\mathrm{N} 8-\mathrm{H} 14)$ & 121.6 & 0.0 & 0.0 & 0.0 & 0.0 & 0.0 & 0.0 & 0.0 & -0.5 \\
\hline$R_{\mathrm{f}}{ }^{\mathrm{c}} \%$ & & 5.80 & 5.60 & 5.34 & 5.19 & 5.00 & 4.72 & 4.11 & 3.08 \\
\hline
\end{tabular}

a Bond lengths and corresponding deviations in $\AA$ and bond angles in degrees.

${ }^{\mathrm{b}}$ Difference between the respective refined and starting (CCSD(T)/cc-pwCVTZ) values.

${ }^{\mathrm{c}}$ Factor of disagreement between model and experimental $s M(s)$ functions. 
Table S11: Deviations of the refined molecular parameters of $\mathrm{PZA}^{\mathrm{a}}$ from their starting $\mathrm{CCSD}(\mathrm{T})$ values for different values of regularizaton parameter $\alpha$. Total intensities $I(s)$ obtained from LMSU GED data were averaged in the refinement procedure.

\begin{tabular}{|c|c|c|c|c|c|c|c|c|c|}
\hline \multirow{2}{*}{ Parameter } & \multirow{2}{*}{$\operatorname{CCSD}(\mathrm{T})$} & \multicolumn{8}{|c|}{ GED } \\
\hline & & $\Delta^{\mathrm{b}}$ & $\Delta$ & $\Delta$ & $\Delta$ & $\Delta$ & $\Delta$ & $\Delta$ & $\Delta$ \\
\hline$\alpha$ & 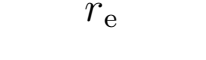 & 150.0 & 100.0 & 80.0 & 60.0 & 40.0 & 20.0 & 1.0 & 0.1 \\
\hline$r(\mathrm{C} 1-\mathrm{N} 2)$ & 1.337 & 0.000 & 0.000 & 0.000 & 0.000 & 0.000 & 0.000 & -0.003 & -0.015 \\
\hline$r(\mathrm{C} 1-\mathrm{C} 6)$ & 1.394 & 0.000 & 0.000 & 0.000 & 0.000 & 0.000 & 0.001 & 0.002 & -0.008 \\
\hline$r(\mathrm{C} 1-\mathrm{C} 7)$ & 1.505 & 0.000 & -0.001 & -0.001 & -0.001 & -0.002 & -0.003 & -0.011 & -0.006 \\
\hline$r(\mathrm{~N} 2-\mathrm{C} 3)$ & 1.335 & 0.000 & 0.000 & 0.000 & 0.000 & 0.000 & 0.000 & 0.003 & 0.004 \\
\hline$r(\mathrm{C} 3-\mathrm{C} 4)$ & 1.393 & 0.000 & 0.000 & 0.000 & 0.000 & 0.001 & 0.001 & 0.014 & 0.021 \\
\hline$r(\mathrm{C} 3-\mathrm{H} 10)$ & 1.082 & 0.000 & 0.000 & 0.000 & 0.000 & 0.000 & 0.000 & 0.002 & 0.005 \\
\hline$r(\mathrm{C} 4-\mathrm{N} 5)$ & 1.337 & 0.000 & 0.000 & 0.000 & 0.000 & 0.000 & 0.001 & 0.005 & 0.039 \\
\hline$r(\mathrm{C} 4-\mathrm{H} 11)$ & 1.082 & 0.000 & 0.000 & 0.000 & 0.000 & 0.000 & 0.000 & -0.002 & -0.020 \\
\hline$r(\mathrm{~N} 5-\mathrm{C} 6)$ & 1.336 & 0.000 & 0.000 & 0.001 & 0.001 & 0.001 & 0.001 & 0.001 & -0.011 \\
\hline$r(\mathrm{C} 6-\mathrm{H} 12)$ & 1.080 & 0.000 & 0.000 & 0.000 & 0.000 & 0.000 & 0.000 & 0.000 & 0.005 \\
\hline$r(\mathrm{C} 7-\mathrm{N} 8)$ & 1.350 & 0.000 & 0.000 & 0.000 & 0.000 & -0.001 & -0.001 & -0.009 & -0.013 \\
\hline$r(\mathrm{C} 7-\mathrm{O} 9)$ & 1.219 & 0.000 & 0.000 & 0.001 & 0.001 & 0.001 & 0.002 & 0.006 & 0.008 \\
\hline$r(\mathrm{~N} 8-\mathrm{H} 13)$ & 1.003 & 0.000 & 0.000 & 0.000 & 0.000 & 0.000 & 0.000 & 0.003 & 0.014 \\
\hline$r(\mathrm{~N} 8-\mathrm{H} 14)$ & 1.002 & 0.000 & 0.000 & 0.000 & 0.000 & 0.000 & 0.000 & 0.001 & 0.020 \\
\hline$\angle(\mathrm{N} 2-\mathrm{C} 1-\mathrm{C} 6)$ & 122.2 & -0.1 & -0.1 & -0.1 & -0.1 & -0.2 & -0.2 & -0.9 & -1.0 \\
\hline$\angle(\mathrm{N} 2-\mathrm{C} 1-\mathrm{C} 7)$ & 118.5 & 0.0 & 0.0 & 0.0 & 0.0 & 0.0 & -0.1 & 0.5 & 0.3 \\
\hline$\angle(\mathrm{C} 1-\mathrm{N} 2-\mathrm{C} 3)$ & 115.9 & 0.0 & 0.0 & 0.1 & 0.1 & 0.1 & 0.1 & 0.6 & 1.5 \\
\hline$\angle(\mathrm{C} 6-\mathrm{C} 1-\mathrm{C} 7)$ & 119.3 & 0.1 & 0.1 & 0.2 & 0.2 & 0.2 & 0.3 & 0.4 & 0.6 \\
\hline$\angle(\mathrm{C} 1-\mathrm{C} 6-\mathrm{N} 5)$ & 122.0 & 0.1 & 0.1 & 0.1 & 0.1 & 0.2 & 0.2 & 1.2 & 2.7 \\
\hline$\angle(\mathrm{C} 1-\mathrm{C} 6-\mathrm{H} 12)$ & 119.6 & -0.1 & -0.1 & -0.1 & -0.2 & -0.2 & -0.2 & -1.4 & -3.8 \\
\hline$\angle(\mathrm{C} 1-\mathrm{C} 7-\mathrm{N} 8)$ & 113.8 & 0.0 & 0.0 & 0.0 & 0.0 & 0.0 & 0.0 & 0.2 & 0.3 \\
\hline$\angle(\mathrm{C} 1-\mathrm{C} 7-\mathrm{O} 9)$ & 121.2 & 0.0 & 0.0 & 0.0 & 0.0 & 0.0 & 0.0 & -0.7 & -1.0 \\
\hline$\angle(\mathrm{N} 2-\mathrm{C} 3-\mathrm{C} 4)$ & 121.9 & 0.0 & 0.0 & 0.0 & 0.0 & 0.0 & 0.1 & 0.1 & -0.7 \\
\hline$\angle(\mathrm{N} 2-\mathrm{C} 3-\mathrm{H} 10)$ & 117.2 & 0.0 & 0.0 & 0.0 & 0.0 & 0.0 & 0.0 & 0.0 & -0.6 \\
\hline$\angle(\mathrm{C} 4-\mathrm{C} 3-\mathrm{H} 10)$ & 120.9 & 0.0 & 0.0 & 0.0 & 0.0 & 0.0 & -0.1 & 0.0 & 1.2 \\
\hline$\angle(\mathrm{C} 3-\mathrm{C} 4-\mathrm{N} 5)$ & 122.5 & 0.0 & 0.0 & 0.0 & 0.0 & 0.0 & -0.1 & -0.6 & -1.0 \\
\hline$\angle(\mathrm{C} 3-\mathrm{C} 4-\mathrm{H} 11)$ & 120.5 & 0.0 & 0.0 & 0.0 & 0.0 & 0.0 & 0.0 & 0.0 & -0.1 \\
\hline$\angle(\mathrm{N} 5-\mathrm{C} 4-\mathrm{H} 11)$ & 117.0 & 0.0 & 0.0 & 0.0 & 0.0 & 0.0 & 0.1 & 0.6 & 1.0 \\
\hline$\angle(\mathrm{C} 4-\mathrm{N} 5-\mathrm{C} 6)$ & 115.6 & 0.0 & -0.1 & -0.1 & -0.1 & -0.1 & -0.1 & -0.4 & -1.5 \\
\hline$\angle(\mathrm{N} 5-\mathrm{C} 6-\mathrm{H} 12)$ & 118.3 & 0.0 & 0.0 & 0.0 & 0.0 & 0.0 & 0.0 & 0.2 & 1.1 \\
\hline$\angle(\mathrm{N} 8-\mathrm{C} 7-\mathrm{O} 9)$ & 125.0 & 0.0 & 0.0 & 0.0 & 0.0 & 0.0 & 0.0 & 0.5 & 0.7 \\
\hline$\angle(\mathrm{C} 7-\mathrm{N} 8-\mathrm{H} 13)$ & 119.4 & 0.0 & 0.0 & 0.0 & 0.0 & 0.0 & 0.0 & 0.1 & 0.6 \\
\hline$\angle(\mathrm{C} 7-\mathrm{N} 8-\mathrm{H} 14)$ & 119.0 & 0.0 & 0.0 & 0.0 & 0.0 & 0.0 & 0.0 & -0.2 & -0.7 \\
\hline$\angle(\mathrm{H} 13-\mathrm{N} 8-\mathrm{H} 14)$ & 121.6 & 0.0 & 0.0 & 0.0 & 0.0 & 0.0 & 0.0 & 0.1 & 0.0 \\
\hline$R_{\mathrm{f}}{ }^{\mathrm{c}} \%$ & & 4.53 & 4.51 & 4.49 & 4.46 & 4.41 & 4.31 & 3.92 & 3.94 \\
\hline
\end{tabular}

${ }^{\text {a }}$ Bond lengths and corresponding deviations in $\AA$ and bond angles in degrees.

b Difference between the respective refined and starting (CCSD(T)/cc-pwCVTZ) values.

${ }^{\mathrm{c}}$ Factor of disagreement between model and experimental $s M(s)$ functions. 
Table S12: Deviations of the refined molecular parameters of $\mathrm{PZA}^{\mathrm{a}}$ from their starting $\mathrm{CCSD}(\mathrm{T})$ values for different values of regularizaton parameter $\alpha$. Total intensities $I(s)$ obtained from ISUCT GED data were averaged in the refinement procedure.

\begin{tabular}{|c|c|c|c|c|c|c|c|c|}
\hline \multirow{2}{*}{ Parameter } & \multirow{2}{*}{$\operatorname{CCSD}(\mathrm{T})$} & \multicolumn{7}{|c|}{ GED } \\
\hline & & $\Delta^{\mathrm{b}}$ & $\Delta$ & $\Delta$ & $\Delta$ & $\Delta$ & $\Delta$ & $\Delta$ \\
\hline$\alpha$ & & 150.0 & 100.0 & 80.0 & 60.0 & 40.0 & 20.0 & 1.0 \\
\hline$r(\mathrm{C} 1-\mathrm{N} 2)$ & 1.337 & -0.003 & -0.003 & -0.003 & -0.003 & -0.003 & 0.000 & -0.064 \\
\hline$r(\mathrm{C} 1-\mathrm{C} 6)$ & 1.394 & -0.001 & -0.001 & -0.001 & -0.001 & -0.001 & 0.001 & 0.016 \\
\hline$r(\mathrm{C} 1-\mathrm{C} 7)$ & 1.505 & -0.004 & -0.005 & -0.006 & -0.006 & -0.008 & -0.007 & -0.002 \\
\hline$r(\mathrm{~N} 2-\mathrm{C} 3)$ & 1.335 & -0.002 & -0.002 & -0.002 & -0.002 & -0.002 & -0.007 & -0.010 \\
\hline$r(\mathrm{C} 3-\mathrm{C} 4)$ & 1.393 & -0.001 & -0.001 & -0.001 & -0.001 & -0.001 & -0.004 & -0.030 \\
\hline$r(\mathrm{C} 3-\mathrm{H} 10)$ & 1.082 & 0.001 & 0.001 & 0.002 & 0.002 & 0.002 & 0.002 & -0.014 \\
\hline$r(\mathrm{C} 4-\mathrm{N} 5)$ & 1.337 & -0.003 & -0.003 & -0.003 & -0.004 & -0.004 & -0.008 & 0.054 \\
\hline$r(\mathrm{C} 4-\mathrm{H} 11)$ & 1.082 & 0.001 & 0.002 & 0.002 & 0.002 & 0.002 & 0.003 & -0.013 \\
\hline$r(\mathrm{~N} 5-\mathrm{C} 6)$ & 1.336 & -0.002 & -0.002 & -0.002 & -0.001 & -0.001 & 0.000 & 0.014 \\
\hline$r(\mathrm{C} 6-\mathrm{H} 12)$ & 1.080 & 0.001 & 0.002 & 0.002 & 0.002 & 0.002 & 0.002 & -0.020 \\
\hline$r(\mathrm{C} 7-\mathrm{N} 8)$ & 1.350 & -0.003 & -0.003 & -0.003 & -0.004 & -0.004 & 0.000 & -0.011 \\
\hline$r(\mathrm{C} 7-\mathrm{O} 9)$ & 1.219 & -0.007 & -0.007 & -0.008 & -0.008 & -0.008 & -0.010 & -0.010 \\
\hline$r(\mathrm{~N} 8-\mathrm{H} 13)$ & 1.003 & -0.001 & -0.001 & -0.001 & -0.001 & -0.002 & -0.003 & -0.099 \\
\hline$r(\mathrm{~N} 8-\mathrm{H} 14)$ & 1.002 & -0.001 & -0.001 & -0.001 & -0.001 & -0.002 & -0.005 & -0.009 \\
\hline$\angle(\mathrm{N} 2-\mathrm{C} 1-\mathrm{C} 6)$ & 122.2 & -0.2 & -0.2 & -0.2 & -0.3 & -0.3 & 0.1 & -1.3 \\
\hline$\angle(\mathrm{N} 2-\mathrm{C} 1-\mathrm{C} 7)$ & 118.5 & 0.0 & 0.1 & 0.1 & 0.2 & 0.3 & 0.3 & 3.3 \\
\hline$\angle(\mathrm{C} 1-\mathrm{N} 2-\mathrm{C} 3)$ & 115.9 & 0.1 & 0.1 & 0.2 & 0.2 & 0.3 & 0.3 & 4.0 \\
\hline$\angle(\mathrm{C} 6-\mathrm{C} 1-\mathrm{C} 7)$ & 119.3 & 0.1 & 0.1 & 0.1 & 0.1 & 0.1 & -0.3 & -2.0 \\
\hline$\angle(\mathrm{C} 1-\mathrm{C} 6-\mathrm{N} 5)$ & 122.0 & 0.1 & 0.1 & 0.1 & 0.1 & 0.1 & -0.9 & 1.4 \\
\hline$\angle(\mathrm{C} 1-\mathrm{C} 6-\mathrm{H} 12)$ & 119.6 & -0.2 & -0.2 & -0.2 & -0.2 & -0.2 & 0.8 & -2.4 \\
\hline$\angle(\mathrm{C} 1-\mathrm{C} 7-\mathrm{N} 8)$ & 113.8 & 0.2 & 0.3 & 0.3 & 0.4 & 0.5 & 0.8 & -1.1 \\
\hline$\angle(\mathrm{C} 1-\mathrm{C} 7-\mathrm{O} 9)$ & 121.2 & 0.1 & 0.1 & 0.2 & 0.2 & 0.2 & 0.4 & 1.5 \\
\hline$\angle(\mathrm{N} 2-\mathrm{C} 3-\mathrm{C} 4)$ & 121.9 & 0.0 & 0.0 & 0.0 & 0.0 & -0.1 & -0.2 & -1.8 \\
\hline$\angle(\mathrm{N} 2-\mathrm{C} 3-\mathrm{H} 10)$ & 117.2 & 0.0 & 0.0 & 0.0 & 0.1 & 0.1 & 0.1 & 1.4 \\
\hline$\angle(\mathrm{C} 4-\mathrm{C} 3-\mathrm{H} 10)$ & 120.9 & 0.0 & 0.0 & 0.0 & 0.0 & 0.0 & 0.1 & 0.4 \\
\hline$\angle(\mathrm{C} 3-\mathrm{C} 4-\mathrm{N} 5)$ & 122.5 & 0.0 & -0.1 & -0.1 & -0.1 & -0.1 & 0.0 & 1.7 \\
\hline$\angle(\mathrm{C} 3-\mathrm{C} 4-\mathrm{H} 11)$ & 120.5 & 0.0 & 0.0 & -0.1 & -0.1 & -0.1 & -0.2 & -0.5 \\
\hline$\angle(\mathrm{N} 5-\mathrm{C} 4-\mathrm{H} 11)$ & 117.0 & 0.1 & 0.1 & 0.1 & 0.2 & 0.2 & 0.1 & -1.2 \\
\hline$\angle(\mathrm{C} 4-\mathrm{N} 5-\mathrm{C} 6)$ & 115.6 & 0.0 & 0.0 & 0.0 & 0.1 & 0.1 & 0.7 & -4.0 \\
\hline$\angle(\mathrm{N} 5-\mathrm{C} 6-\mathrm{H} 12)$ & 118.3 & 0.0 & 0.0 & 0.1 & 0.1 & 0.1 & 0.2 & 1.0 \\
\hline$\angle(\mathrm{N} 8-\mathrm{C} 7-\mathrm{O} 9)$ & 125.0 & -0.3 & -0.4 & -0.5 & -0.6 & -0.7 & -1.2 & -0.4 \\
\hline$\angle(\mathrm{C} 7-\mathrm{N} 8-\mathrm{H} 13)$ & 119.4 & 0.0 & 0.0 & 0.0 & 0.0 & 0.0 & 0.0 & 0.2 \\
\hline$\angle(\mathrm{C} 7-\mathrm{N} 8-\mathrm{H} 14)$ & 119.0 & 0.0 & 0.0 & 0.0 & 0.0 & 0.0 & 0.0 & 2.4 \\
\hline$\angle(\mathrm{H} 13-\mathrm{N} 8-\mathrm{H} 14)$ & 121.6 & 0.0 & 0.0 & 0.0 & 0.0 & 0.0 & 0.0 & -2.7 \\
\hline$R_{\mathrm{f}},{ }^{\mathrm{c}} \%$ & & 6.19 & 6.10 & 6.06 & 5.90 & 5.78 & 5.85 & 5.39 \\
\hline
\end{tabular}

a Bond lengths and corresponding deviations in $\AA$ and bond angles in degrees.

b Difference between the respective refined and starting (CCSD $(\mathrm{T}) / \mathrm{cc}-\mathrm{pwCVTZ})$ values.

${ }^{\mathrm{c}}$ Factor of disagreement between model and experimental $s M(s)$ functions. 
Table S13: Computed (anharmonic) and observed (xenon matrix, $20 \mathrm{~K}$ ) vibrational frequencies of PZA and root-mean-square deviations (all values in $\mathrm{cm}^{-1}$ )

\begin{tabular}{|c|c|c|c|c|c|c|c|c|}
\hline expt. & B3LYP & O3LYP & X3LYP & PW6B95 & PBE0 & B3PW91 & TPSSh & BP86 \\
\hline Ref. [2] & \multicolumn{7}{|c|}{ def2-TZVP } & def2-SV $(\mathrm{P})$ \\
\hline \multicolumn{9}{|c|}{$\mathrm{A}^{\prime}$} \\
\hline 3530 & 3522 & 3537 & 3529 & 3582 & 3564 & 3545 & 3493 & 3367 \\
\hline 3404 & 3407 & 3417 & 3413 & 3465 & 3440 & 3422 & 3369 & 3246 \\
\hline 3075 & 3067 & 3061 & 3076 & 3117 & 3076 & 3077 & 3062 & 2962 \\
\hline 3058 & 3043 & 3033 & 3051 & 3090 & 3059 & 3055 & 3041 & 2945 \\
\hline 3049 & 2999 & 2986 & 3008 & 3047 & 3040 & 3013 & 2995 & 2909 \\
\hline 1716 & 1726 & 1732 & 1733 & 1766 & 1773 & 1750 & 1715 & 1724 \\
\hline 1570 & 1576 & 1568 & 1581 & 1608 & 1604 & 1585 & 1573 & 1532 \\
\hline 1554 & 1552 & 1539 & 1551 & 1575 & 1577 & 1558 & 1544 & 1513 \\
\hline 1551 & 1538 & 1538 & 1546 & 1664 & 1562 & 1549 & 1526 & 1506 \\
\hline \multirow[t]{3}{*}{1470} & 1473 & 1462 & 1478 & 1491 & 1488 & 1477 & 1466 & 1428 \\
\hline & 1413 & 1399 & 1416 & 1435 & 1428 & 1416 & 1404 & 1374 \\
\hline & 1347 & 1338 & 1352 & 1367 & 1363 & 1355 & 1342 & 1324 \\
\hline 1312 & 1292 & 1281 & 1296 & 1301 & 1297 & 1291 & 1286 & 1240 \\
\hline \multirow[t]{2}{*}{1205} & 1197 & 1217 & 1199 & 1221 & 1234 & 1220 & 1203 & 1247 \\
\hline & 1169 & 1174 & 1172 & 1198 & 1191 & 1180 & 1173 & 1144 \\
\hline \multirow[t]{2}{*}{1164} & 1164 & 1161 & 1168 & 1176 & 1169 & 1177 & 1166 & 1133 \\
\hline & 1088 & 1088 & 1091 & 1178 & 1103 & 1091 & 1087 & 1078 \\
\hline \multirow[t]{2}{*}{1047} & 1054 & 1050 & 1056 & 1073 & 1067 & 1059 & 1049 & 1028 \\
\hline & 1024 & 1013 & 1027 & 1029 & 1029 & 1022 & 1013 & 988 \\
\hline 812 & 799 & 788 & 802 & 806 & 806 & 800 & 789 & 779 \\
\hline 641 & 645 & 638 & 647 & 670 & 647 & 643 & 634 & 625 \\
\hline \multirow[t]{3}{*}{605} & 607 & 597 & 609 & 603 & 605 & 603 & 591 & 587 \\
\hline & 374 & 367 & 373 & 437 & 377 & 372 & 365 & 368 \\
\hline & 211 & 206 & 211 & 282 & 214 & 209 & 203 & 211 \\
\hline \multicolumn{9}{|c|}{$\mathrm{A}^{\prime \prime}$} \\
\hline \multirow[t]{4}{*}{953} & 992 & 984 & 994 & 1007 & 1001 & 993 & 987 & 939 \\
\hline & 969 & 957 & 972 & 980 & 973 & 969 & 961 & 927 \\
\hline & 876 & 867 & 879 & 887 & 882 & 878 & 871 & 841 \\
\hline & 784 & 775 & 786 & 791 & 787 & 783 & 775 & 759 \\
\hline 721 & 731 & 724 & 734 & 737 & 740 & 734 & 722 & 709 \\
\hline 605 & 586 & 582 & 589 & 600 & 598 & 593 & 587 & 603 \\
\hline \multirow[t]{5}{*}{439} & 457 & 442 & 460 & 457 & 460 & 456 & 445 & 456 \\
\hline & 390 & 374 & 392 & 390 & 391 & 385 & 373 & 406 \\
\hline & 409 & 408 & 414 & 428 & 405 & 409 & 406 & 392 \\
\hline & 151 & 147 & 155 & 153 & 160 & 151 & 148 & 167 \\
\hline & 76 & 73 & 82 & 77 & 82 & 80 & 81 & 73 \\
\hline \multicolumn{9}{|c|}{ RMSDs } \\
\hline & 17 & 21 & 16 & 39 & 24 & 17 & 21 & 72 \\
\hline
\end{tabular}


Table S14: GED terms (in $\AA$ ) for the refinement of the UBi GED data. Molecular intensities $s M(s)$ were averaged in the refinement procedure.

\begin{tabular}{|c|c|c|c|c|c|c|c|c|}
\hline Atom 1 & Atom 2 & $r_{\mathrm{a}}$ & $l_{\text {calc. }}$ & $l_{\text {exp. }}$ & $\sigma_{\mathrm{LS}}$ & $\sigma_{\exp }$ & $\left(r_{\mathrm{e}}-r_{\mathrm{a}}\right)$ & Group \\
\hline N8 & H14 & 1.022475 & 0.070100 & 0.072866 & $1.7 \mathrm{e}-03$ & $2.6 \mathrm{e}-03$ & -0.017100 & 100 \\
\hline N8 & H13 & 1.022734 & 0.070300 & 0.073073 & $1.7 \mathrm{e}-03$ & $2.6 \mathrm{e}-03$ & -0.017100 & 100 \\
\hline C6 & H12 & 1.098059 & 0.075300 & 0.078271 & $1.9 \mathrm{e}-03$ & $2.8 \mathrm{e}-03$ & -0.015900 & 100 \\
\hline C3 & H10 & 1.099861 & 0.075700 & 0.078686 & $1.9 \mathrm{e}-03$ & $2.8 \mathrm{e}-03$ & -0.016000 & 100 \\
\hline $\mathrm{C} 4$ & H11 & 1.100329 & 0.075700 & 0.078686 & $1.9 \mathrm{e}-03$ & $2.8 \mathrm{e}-03$ & -0.016000 & 100 \\
\hline $\mathrm{C} 7$ & O9 & 1.222243 & 0.038200 & 0.037261 & $7.4 \mathrm{e}-04$ & $7.9 \mathrm{e}-04$ & -0.002300 & 101 \\
\hline $\mathrm{N} 2$ & C3 & 1.335137 & 0.044600 & 0.043504 & $8.6 \mathrm{e}-04$ & $9.2 \mathrm{e}-04$ & -0.006300 & 101 \\
\hline N5 & C6 & 1.338261 & 0.044600 & 0.043504 & $8.6 \mathrm{e}-04$ & $9.2 \mathrm{e}-04$ & -0.005600 & 101 \\
\hline $\mathrm{C} 4$ & N5 & 1.338811 & 0.044800 & 0.043699 & $8.7 \mathrm{e}-04$ & $9.2 \mathrm{e}-04$ & -0.006200 & 101 \\
\hline $\mathrm{C} 1$ & $\mathrm{~N} 2$ & 1.338423 & 0.045000 & 0.043894 & $8.7 \mathrm{e}-04$ & $9.3 \mathrm{e}-04$ & -0.005700 & 101 \\
\hline $\mathrm{C} 7$ & N8 & 1.364939 & 0.044500 & 0.043406 & $8.6 \mathrm{e}-04$ & $9.2 \mathrm{e}-04$ & -0.017700 & 101 \\
\hline C3 & $\mathrm{C} 4$ & 1.398940 & 0.046700 & 0.045552 & $9.1 \mathrm{e}-04$ & $9.6 \mathrm{e}-04$ & -0.007700 & 101 \\
\hline $\mathrm{C} 1$ & C6 & 1.405920 & 0.046900 & 0.045747 & $9.1 \mathrm{e}-04$ & $9.6 \mathrm{e}-04$ & -0.008300 & 101 \\
\hline $\mathrm{C} 1$ & $\mathrm{C} 7$ & 1.510916 & 0.052300 & 0.051014 & $1.0 \mathrm{e}-03$ & $1.1 \mathrm{e}-03$ & -0.009500 & 101 \\
\hline H13 & H14 & 1.747698 & 0.117100 & 0.110564 & $3.1 \mathrm{e}-03$ & $5.5 \mathrm{e}-03$ & 0.007800 & 102 \\
\hline $\mathrm{C} 7$ & H14 & 2.028778 & 0.103900 & 0.098101 & $2.8 \mathrm{e}-03$ & $4.9 \mathrm{e}-03$ & 0.006100 & 102 \\
\hline $\mathrm{C} 7$ & H13 & 2.038023 & 0.102800 & 0.097062 & $2.7 \mathrm{e}-03$ & $4.8 \mathrm{e}-03$ & 0.000300 & 102 \\
\hline $\mathrm{N} 2$ & H10 & 2.076921 & 0.096300 & 0.090925 & $2.6 \mathrm{e}-03$ & $4.5 \mathrm{e}-03$ & -0.013500 & 102 \\
\hline N5 & H11 & 2.079426 & 0.096300 & 0.090925 & $2.6 \mathrm{e}-03$ & $4.5 \mathrm{e}-03$ & -0.013900 & 102 \\
\hline N5 & H12 & 2.088763 & 0.096600 & 0.091208 & $2.6 \mathrm{e}-03$ & $4.6 \mathrm{e}-03$ & -0.011300 & 102 \\
\hline C1 & H12 & 2.165988 & 0.098600 & 0.093097 & $2.6 \mathrm{e}-03$ & $4.6 \mathrm{e}-03$ & -0.018000 & 102 \\
\hline C3 & H11 & 2.170827 & 0.098200 & 0.092719 & $2.6 \mathrm{e}-03$ & $4.6 \mathrm{e}-03$ & -0.016100 & 102 \\
\hline $\mathrm{C} 4$ & H10 & 2.173207 & 0.098100 & 0.092625 & $2.6 \mathrm{e}-03$ & $4.6 \mathrm{e}-03$ & -0.015800 & 102 \\
\hline $\mathrm{C} 4$ & C6 & 2.265160 & 0.054200 & 0.060563 & 8.7e-04 & $9.3 \mathrm{e}-04$ & -0.010700 & 103 \\
\hline $\mathrm{C} 1$ & C3 & 2.268514 & 0.054100 & 0.060451 & 8.7e-04 & $9.3 \mathrm{e}-04$ & -0.010800 & 103 \\
\hline N8 & O9 & 2.292661 & 0.055500 & 0.062016 & $8.9 \mathrm{e}-04$ & $9.5 \mathrm{e}-04$ & -0.011600 & 103 \\
\hline $\mathrm{N} 2$ & H13 & 2.289650 & 0.195700 & 0.218676 & $3.1 \mathrm{e}-03$ & $3.4 \mathrm{e}-03$ & -0.047500 & 103 \\
\hline N2 & $\mathrm{C} 4$ & 2.387143 & 0.054600 & 0.061010 & $8.7 \mathrm{e}-04$ & $9.4 \mathrm{e}-04$ & -0.008000 & 103 \\
\hline $\mathrm{N} 2$ & C6 & 2.393707 & 0.054800 & 0.061234 & $8.8 \mathrm{e}-04$ & $9.4 \mathrm{e}-04$ & -0.007500 & 103 \\
\hline $\mathrm{C} 1$ & N5 & 2.398133 & 0.054700 & 0.061122 & $8.8 \mathrm{e}-04$ & $9.4 \mathrm{e}-04$ & -0.008000 & 103 \\
\hline C3 & N5 & 2.394883 & 0.054700 & 0.061122 & $8.8 \mathrm{e}-04$ & $9.4 \mathrm{e}-04$ & -0.007400 & 103 \\
\hline $\mathrm{C} 1$ & O9 & 2.378788 & 0.063500 & 0.070955 & $1.0 \mathrm{e}-03$ & $1.1 \mathrm{e}-03$ & -0.006100 & 103 \\
\hline C1 & N8 & 2.412973 & 0.065500 & 0.073190 & $1.0 \mathrm{e}-03$ & $1.1 \mathrm{e}-03$ & -0.026700 & 103 \\
\hline N2 & $\mathrm{C} 7$ & 2.444921 & 0.065800 & 0.073525 & $1.1 \mathrm{e}-03$ & $1.1 \mathrm{e}-03$ & -0.010600 & 103 \\
\hline H10 & H11 & 2.515697 & 0.158500 & 0.177108 & $2.5 \mathrm{e}-03$ & $2.7 \mathrm{e}-03$ & -0.019100 & 103 \\
\hline C6 & $\mathrm{C} 7$ & 2.524145 & 0.070200 & 0.078442 & $1.1 \mathrm{e}-03$ & $1.2 \mathrm{e}-03$ & -0.014200 & 103 \\
\hline $\mathrm{C} 1$ & H13 & 2.508964 & 0.154700 & 0.172862 & $2.5 \mathrm{e}-03$ & $2.7 \mathrm{e}-03$ & -0.015300 & 103 \\
\hline O9 & H14 & 2.526027 & 0.147100 & 0.164370 & $2.4 \mathrm{e}-03$ & $2.5 \mathrm{e}-03$ & 0.017000 & 103 \\
\hline O9 & H12 & 2.569048 & 0.187300 & 0.209289 & $3.0 \mathrm{e}-03$ & $3.2 \mathrm{e}-03$ & -0.042500 & 103 \\
\hline C1 & $\mathrm{C} 4$ & 2.662908 & 0.060000 & 0.058659 & $1.4 \mathrm{e}-03$ & $1.8 \mathrm{e}-03$ & -0.011700 & 104 \\
\hline C3 & C6 & 2.664063 & 0.060400 & 0.059050 & $1.4 \mathrm{e}-03$ & $1.8 \mathrm{e}-03$ & -0.010800 & 104 \\
\hline N2 & N8 & 2.717286 & 0.105100 & 0.102751 & $2.5 \mathrm{e}-03$ & $3.2 \mathrm{e}-03$ & -0.042800 & 104 \\
\hline $\mathrm{C} 7$ & H12 & 2.725202 & 0.143100 & 0.139902 & $3.4 \mathrm{e}-03$ & $4.3 \mathrm{e}-03$ & -0.022200 & 104 \\
\hline $\mathrm{N} 2$ & N5 & 2.814189 & 0.064600 & 0.063156 & $1.5 \mathrm{e}-03$ & $1.9 \mathrm{e}-03$ & -0.004200 & 104 \\
\hline C6 & O9 & 2.852788 & 0.108700 & 0.106271 & $2.6 \mathrm{e}-03$ & $3.3 \mathrm{e}-03$ & -0.021300 & 104 \\
\hline O9 & H13 & 3.149544 & 0.097300 & 0.096063 & $2.8 \mathrm{e}-03$ & $9.4 \mathrm{e}-03$ & 0.010100 & 105 \\
\hline
\end{tabular}


Table S14 - continued from previous page

\begin{tabular}{|c|c|c|c|c|c|c|c|c|}
\hline Atom 1 & Atom 2 & $r_{\mathrm{a}}$ & $l_{\text {calc. }}$ & $l_{\text {exp. }}$ & $\sigma_{\mathrm{LS}}$ & $\sigma_{\text {exp }}$ & $\left(r_{\mathrm{e}}-r_{\mathrm{a}}\right)$ & Group \\
\hline C6 & H11 & 3.251631 & 0.093300 & 0.092114 & $2.7 \mathrm{e}-03$ & $9.1 \mathrm{e}-03$ & -0.017100 & 105 \\
\hline $\mathrm{C} 1$ & H10 & 3.254004 & 0.093300 & 0.092114 & $2.7 \mathrm{e}-03$ & $9.1 \mathrm{e}-03$ & -0.017100 & 105 \\
\hline $\mathrm{C} 4$ & H12 & 3.256965 & 0.093200 & 0.092015 & $2.7 \mathrm{e}-03$ & $9.0 \mathrm{e}-03$ & -0.016100 & 105 \\
\hline $\mathrm{C} 1$ & H14 & 3.320408 & 0.100100 & 0.098827 & $2.9 \mathrm{e}-03$ & $9.7 \mathrm{e}-03$ & 0.000100 & 105 \\
\hline N2 & H12 & 3.368197 & 0.094200 & 0.093002 & $2.7 \mathrm{e}-03$ & $9.1 \mathrm{e}-03$ & -0.016100 & 105 \\
\hline N2 & H11 & 3.369370 & 0.094100 & 0.092904 & $2.7 \mathrm{e}-03$ & $9.1 \mathrm{e}-03$ & -0.015500 & 105 \\
\hline N5 & H10 & 3.377511 & 0.094200 & 0.093002 & $2.7 \mathrm{e}-03$ & $9.1 \mathrm{e}-03$ & -0.015000 & 105 \\
\hline $\mathrm{N} 2$ & O9 & 3.539499 & 0.066000 & 0.069589 & $1.7 \mathrm{e}-03$ & $2.9 \mathrm{e}-03$ & 0.003000 & 106 \\
\hline C3 & H13 & 3.562817 & 0.204100 & 0.215198 & $5.3 \mathrm{e}-03$ & $9.0 \mathrm{e}-03$ & -0.044700 & 106 \\
\hline C3 & $\mathrm{C} 7$ & 3.650899 & 0.067000 & 0.070643 & $1.8 \mathrm{e}-03$ & $3.0 \mathrm{e}-03$ & -0.013000 & 106 \\
\hline C6 & N8 & 3.687997 & 0.071900 & 0.075810 & $1.9 \mathrm{e}-03$ & $3.2 \mathrm{e}-03$ & -0.016000 & 106 \\
\hline $\mathrm{N} 2$ & H14 & 3.691320 & 0.133800 & 0.141076 & $3.5 \mathrm{e}-03$ & $5.9 \mathrm{e}-03$ & -0.013300 & 106 \\
\hline $\mathrm{C} 1$ & H11 & 3.753592 & 0.093100 & 0.098163 & $2.4 \mathrm{e}-03$ & $4.1 \mathrm{e}-03$ & -0.018200 & 106 \\
\hline C3 & H12 & 3.752360 & 0.093100 & 0.098163 & $2.4 \mathrm{e}-03$ & $4.1 \mathrm{e}-03$ & -0.017500 & 106 \\
\hline C6 & H10 & 3.754401 & 0.093300 & 0.098373 & $2.4 \mathrm{e}-03$ & $4.1 \mathrm{e}-03$ & -0.017400 & 106 \\
\hline N5 & $\mathrm{C} 7$ & 3.761179 & 0.069500 & 0.073279 & $1.8 \mathrm{e}-03$ & $3.1 \mathrm{e}-03$ & -0.011800 & 106 \\
\hline C6 & H13 & 3.892036 & 0.155500 & 0.163811 & $4.1 \mathrm{e}-03$ & 7.3e-03 & -0.004600 & 107 \\
\hline H10 & H13 & 3.990344 & 0.237800 & 0.250510 & $6.3 \mathrm{e}-03$ & $1.1 \mathrm{e}-02$ & -0.060600 & 107 \\
\hline C3 & N8 & 4.040605 & 0.109100 & 0.114931 & $2.9 \mathrm{e}-03$ & $5.1 \mathrm{e}-03$ & -0.037400 & 107 \\
\hline N8 & H12 & 4.037255 & 0.142000 & 0.149590 & $3.8 \mathrm{e}-03$ & $6.7 \mathrm{e}-03$ & -0.013300 & 107 \\
\hline H11 & H12 & 4.148529 & 0.128100 & 0.134947 & $3.4 \mathrm{e}-03$ & $6.0 \mathrm{e}-03$ & -0.019200 & 107 \\
\hline $\mathrm{C} 4$ & $\mathrm{C} 7$ & 4.165726 & 0.069800 & 0.073531 & $1.9 \mathrm{e}-03$ & $3.3 \mathrm{e}-03$ & -0.013600 & 107 \\
\hline N5 & O9 & 4.178985 & 0.111200 & 0.117144 & $2.9 \mathrm{e}-03$ & $5.2 \mathrm{e}-03$ & -0.015000 & 107 \\
\hline H12 & H13 & 4.469238 & 0.177000 & 0.182055 & $4.8 \mathrm{e}-03$ & $1.0 \mathrm{e}-02$ & 0.001100 & 108 \\
\hline $\mathrm{C} 7$ & H10 & 4.512298 & 0.110400 & 0.113553 & $3.0 \mathrm{e}-03$ & $6.5 \mathrm{e}-03$ & -0.016300 & 108 \\
\hline C6 & H14 & 4.509960 & 0.112100 & 0.115301 & $3.1 \mathrm{e}-03$ & $6.6 \mathrm{e}-03$ & 0.010800 & 108 \\
\hline N8 & H10 & 4.654549 & 0.160200 & 0.164775 & $4.4 \mathrm{e}-03$ & $9.5 \mathrm{e}-03$ & -0.044500 & 108 \\
\hline C3 & O9 & 4.627646 & 0.074100 & 0.076216 & $2.0 \mathrm{e}-03$ & $4.4 \mathrm{e}-03$ & 0.000400 & 108 \\
\hline $\mathrm{C} 4$ & H13 & 4.633786 & 0.194600 & 0.200158 & $5.3 \mathrm{e}-03$ & $1.1 \mathrm{e}-02$ & -0.027700 & 108 \\
\hline H12 & H14 & 4.702389 & 0.183900 & 0.189152 & $5.0 \mathrm{e}-03$ & $1.1 \mathrm{e}-02$ & 0.011200 & 108 \\
\hline N5 & N8 & 4.790989 & 0.077900 & 0.080125 & $2.1 \mathrm{e}-03$ & $4.6 \mathrm{e}-03$ & -0.014700 & 108 \\
\hline N5 & H13 & 4.794964 & 0.175300 & 0.180306 & $4.8 \mathrm{e}-03$ & $1.0 \mathrm{e}-02$ & -0.007900 & 108 \\
\hline H10 & H12 & 4.840502 & 0.117500 & 0.120856 & $3.2 \mathrm{e}-03$ & $6.9 \mathrm{e}-03$ & -0.021800 & 108 \\
\hline $\mathrm{C} 4$ & O9 & 4.881981 & 0.095500 & 0.098227 & 2.6e-03 & $5.6 \mathrm{e}-03$ & -0.006800 & 108 \\
\hline $\mathrm{C} 4$ & N8 & 4.901023 & 0.094000 & 0.096685 & 2.6e-03 & $5.5 \mathrm{e}-03$ & -0.026700 & 108 \\
\hline C3 & H14 & 5.013652 & 0.137500 & 0.141427 & $3.8 \mathrm{e}-03$ & $8.1 \mathrm{e}-03$ & -0.006800 & 108 \\
\hline $\mathrm{C} 7$ & H11 & 5.253629 & 0.100100 & 0.100382 & $3.0 \mathrm{e}-03$ & $3.1 \mathrm{e}-02$ & -0.017200 & 109 \\
\hline O9 & H10 & 5.569835 & 0.108400 & 0.108706 & $3.2 \mathrm{e}-03$ & $3.4 \mathrm{e}-02$ & 0.001700 & 109 \\
\hline H10 & H14 & 5.598322 & 0.192000 & 0.192541 & $5.7 \mathrm{e}-03$ & $6.0 \mathrm{e}-02$ & -0.014000 & 109 \\
\hline H11 & H13 & 5.629596 & 0.215600 & 0.216208 & $6.4 \mathrm{e}-03$ & $6.7 \mathrm{e}-02$ & -0.033600 & 109 \\
\hline N5 & H14 & 5.680523 & 0.108700 & 0.109006 & $3.2 \mathrm{e}-03$ & $3.4 \mathrm{e}-02$ & 0.015300 & 109 \\
\hline $\mathrm{C} 4$ & H14 & 5.861226 & 0.117800 & 0.118132 & $3.5 \mathrm{e}-03$ & $3.7 \mathrm{e}-02$ & 0.004900 & 109 \\
\hline O9 & H11 & 5.948488 & 0.123600 & 0.123948 & $3.7 \mathrm{e}-03$ & $3.8 \mathrm{e}-02$ & -0.008700 & 109 \\
\hline N8 & H11 & 5.957784 & 0.123500 & 0.123848 & $3.7 \mathrm{e}-03$ & $3.8 \mathrm{e}-02$ & -0.029300 & 109 \\
\hline H11 & H14 & 6.921923 & 0.142700 & 0.143102 & $4.3 \mathrm{e}-03$ & $4.4 \mathrm{e}-02$ & 0.004300 & 109 \\
\hline
\end{tabular}


Table S15: GED terms (in $\AA$ ) for the refinement of the LMSU GED data. Molecular intensities $s M(s)$ were averaged in the refinement procedure.

\begin{tabular}{|c|c|c|c|c|c|c|c|c|}
\hline Atom 1 & Atom 2 & $r_{\mathrm{a}}$ & $l_{\text {calc. }}$ & $l_{\text {exp. }}$ & $\sigma_{\mathrm{LS}}$ & $\sigma_{\exp }$ & $\left(r_{\mathrm{e}}-r_{\mathrm{a}}\right)$ & Group \\
\hline N8 & H14 & 1.018620 & 0.070100 & 0.071064 & $1.0 \mathrm{e}-03$ & $2.1 \mathrm{e}-03$ & -0.016700 & 100 \\
\hline N8 & H13 & 1.020110 & 0.070300 & 0.071267 & $1.0 \mathrm{e}-03$ & $2.1 \mathrm{e}-03$ & -0.016700 & 100 \\
\hline C6 & H12 & 1.096215 & 0.075300 & 0.076336 & $1.1 \mathrm{e}-03$ & $2.2 \mathrm{e}-03$ & -0.015800 & 100 \\
\hline C3 & H10 & 1.097964 & 0.075700 & 0.076741 & $1.1 \mathrm{e}-03$ & $2.3 \mathrm{e}-03$ & -0.015900 & 100 \\
\hline $\mathrm{C} 4$ & H11 & 1.098511 & 0.075700 & 0.076741 & $1.1 \mathrm{e}-03$ & 2.3e-03 & -0.015900 & 100 \\
\hline $\mathrm{C} 7$ & O9 & 1.223731 & 0.038200 & 0.039160 & $4.2 \mathrm{e}-04$ & $4.6 \mathrm{e}-04$ & -0.002400 & 101 \\
\hline $\mathrm{N} 2$ & C3 & 1.342672 & 0.044300 & 0.045413 & $4.9 \mathrm{e}-04$ & $5.3 \mathrm{e}-04$ & -0.006100 & 101 \\
\hline N5 & C6 & 1.343248 & 0.044400 & 0.045516 & $4.9 \mathrm{e}-04$ & $5.3 \mathrm{e}-04$ & -0.005500 & 101 \\
\hline $\mathrm{C} 4$ & N5 & 1.344459 & 0.044500 & 0.045618 & $4.9 \mathrm{e}-04$ & $5.4 \mathrm{e}-04$ & -0.006100 & 101 \\
\hline $\mathrm{C} 1$ & $\mathrm{~N} 2$ & 1.344420 & 0.044700 & 0.045823 & $4.9 \mathrm{e}-04$ & $5.4 \mathrm{e}-04$ & -0.005600 & 101 \\
\hline $\mathrm{C} 7$ & N8 & 1.368253 & 0.044200 & 0.045311 & $4.9 \mathrm{e}-04$ & $5.3 \mathrm{e}-04$ & -0.016600 & 101 \\
\hline C3 & $\mathrm{C} 4$ & 1.404279 & 0.046400 & 0.047566 & $5.1 \mathrm{e}-04$ & $5.6 \mathrm{e}-04$ & -0.007500 & 101 \\
\hline $\mathrm{C} 1$ & C6 & 1.406114 & 0.046600 & 0.047771 & $5.1 \mathrm{e}-04$ & $5.6 \mathrm{e}-04$ & -0.008000 & 101 \\
\hline $\mathrm{C} 1$ & $\mathrm{C} 7$ & 1.515321 & 0.051600 & 0.052897 & $5.7 \mathrm{e}-04$ & $6.2 \mathrm{e}-04$ & -0.009100 & 101 \\
\hline H13 & H14 & 1.745482 & 0.116300 & 0.116623 & $1.7 \mathrm{e}-03$ & $5.3 \mathrm{e}-03$ & 0.005400 & 102 \\
\hline $\mathrm{C} 7$ & H14 & 2.030912 & 0.102500 & 0.102785 & $1.5 \mathrm{e}-03$ & $4.7 \mathrm{e}-03$ & 0.004700 & 102 \\
\hline $\mathrm{C} 7$ & H13 & 2.041121 & 0.101300 & 0.101581 & $1.5 \mathrm{e}-03$ & $4.6 \mathrm{e}-03$ & -0.000600 & 102 \\
\hline $\mathrm{N} 2$ & H10 & 2.082076 & 0.095900 & 0.096166 & $1.4 \mathrm{e}-03$ & $4.4 \mathrm{e}-03$ & -0.013500 & 102 \\
\hline N5 & H11 & 2.082504 & 0.096000 & 0.096267 & $1.4 \mathrm{e}-03$ & $4.4 \mathrm{e}-03$ & -0.013800 & 102 \\
\hline N5 & H12 & 2.091888 & 0.096300 & 0.096567 & $1.4 \mathrm{e}-03$ & $4.4 \mathrm{e}-03$ & -0.011400 & 102 \\
\hline C1 & H12 & 2.163575 & 0.098100 & 0.098372 & $1.5 \mathrm{e}-03$ & $4.5 \mathrm{e}-03$ & -0.017600 & 102 \\
\hline C3 & H11 & 2.174435 & 0.097700 & 0.097971 & $1.5 \mathrm{e}-03$ & $4.4 \mathrm{e}-03$ & -0.015900 & 102 \\
\hline $\mathrm{C} 4$ & H10 & 2.177720 & 0.097600 & 0.097871 & $1.5 \mathrm{e}-03$ & $4.4 \mathrm{e}-03$ & -0.015600 & 102 \\
\hline $\mathrm{C} 4$ & C6 & 2.273566 & 0.053400 & 0.054994 & $5.7 \mathrm{e}-04$ & $6.8 \mathrm{e}-04$ & -0.010600 & 103 \\
\hline $\mathrm{C} 1$ & C3 & 2.278459 & 0.053300 & 0.054891 & $5.7 \mathrm{e}-04$ & $6.8 \mathrm{e}-04$ & -0.010500 & 103 \\
\hline N8 & O9 & 2.293975 & 0.054400 & 0.056024 & $5.8 \mathrm{e}-04$ & $6.9 \mathrm{e}-04$ & -0.011200 & 103 \\
\hline $\mathrm{N} 2$ & H13 & 2.301897 & 0.189000 & 0.194641 & $2.0 \mathrm{e}-03$ & $2.4 \mathrm{e}-03$ & -0.043300 & 103 \\
\hline N2 & $\mathrm{C} 4$ & 2.397632 & 0.053800 & 0.055406 & $5.8 \mathrm{e}-04$ & $6.9 \mathrm{e}-04$ & -0.008000 & 103 \\
\hline $\mathrm{N} 2$ & C6 & 2.401111 & 0.054000 & 0.055612 & $5.8 \mathrm{e}-04$ & $6.9 \mathrm{e}-04$ & -0.007500 & 103 \\
\hline $\mathrm{C} 1$ & N5 & 2.404052 & 0.053800 & 0.055406 & $5.8 \mathrm{e}-04$ & $6.9 \mathrm{e}-04$ & -0.007900 & 103 \\
\hline C3 & N5 & 2.405136 & 0.053900 & 0.055509 & $5.8 \mathrm{e}-04$ & $6.9 \mathrm{e}-04$ & -0.007500 & 103 \\
\hline $\mathrm{C} 1$ & O9 & 2.387114 & 0.061900 & 0.063747 & $6.6 \mathrm{e}-04$ & $7.9 \mathrm{e}-04$ & -0.006100 & 103 \\
\hline C1 & N8 & 2.420222 & 0.063800 & 0.065704 & $6.8 \mathrm{e}-04$ & $8.1 \mathrm{e}-04$ & -0.025000 & 103 \\
\hline N2 & $\mathrm{C} 7$ & 2.456358 & 0.064000 & 0.065910 & $6.9 \mathrm{e}-04$ & $8.2 \mathrm{e}-04$ & -0.010200 & 103 \\
\hline H10 & H11 & 2.521194 & 0.157300 & 0.161995 & $1.7 \mathrm{e}-03$ & $2.0 \mathrm{e}-03$ & -0.018600 & 103 \\
\hline C6 & $\mathrm{C} 7$ & 2.523819 & 0.068300 & 0.070338 & 7.3e-04 & $8.7 \mathrm{e}-04$ & -0.013600 & 103 \\
\hline $\mathrm{C} 1$ & H13 & 2.516569 & 0.151000 & 0.155507 & $1.6 \mathrm{e}-03$ & $1.9 \mathrm{e}-03$ & -0.014500 & 103 \\
\hline O9 & H14 & 2.525718 & 0.143700 & 0.147989 & $1.5 \mathrm{e}-03$ & $1.8 \mathrm{e}-03$ & 0.014700 & 103 \\
\hline O9 & H12 & 2.563013 & 0.181300 & 0.186711 & $1.9 \mathrm{e}-03$ & 2.3e-03 & -0.039200 & 103 \\
\hline C1 & $\mathrm{C} 4$ & 2.671558 & 0.058900 & 0.060013 & $8.3 \mathrm{e}-04$ & $1.4 \mathrm{e}-03$ & -0.011500 & 104 \\
\hline C3 & C6 & 2.674338 & 0.059300 & 0.060421 & $8.3 \mathrm{e}-04$ & $1.4 \mathrm{e}-03$ & -0.010800 & 104 \\
\hline N2 & N8 & 2.729475 & 0.100700 & 0.102604 & $1.4 \mathrm{e}-03$ & $2.4 \mathrm{e}-03$ & -0.039500 & 104 \\
\hline $\mathrm{C} 7$ & H12 & 2.718155 & 0.140300 & 0.142952 & $2.0 \mathrm{e}-03$ & $3.3 \mathrm{e}-03$ & -0.021200 & 104 \\
\hline $\mathrm{N} 2$ & N5 & 2.825772 & 0.063000 & 0.064191 & $8.8 \mathrm{e}-04$ & $1.5 \mathrm{e}-03$ & -0.004500 & 104 \\
\hline C6 & O9 & 2.854843 & 0.104200 & 0.106170 & $1.5 \mathrm{e}-03$ & $2.5 \mathrm{e}-03$ & -0.020000 & 104 \\
\hline O9 & H13 & 3.152231 & 0.095700 & 0.094752 & $1.5 \mathrm{e}-03$ & $7.2 \mathrm{e}-03$ & 0.008000 & 105 \\
\hline
\end{tabular}


Table S15 - continued from previous page

\begin{tabular}{|c|c|c|c|c|c|c|c|c|}
\hline Atom 1 & Atom 2 & $r_{\mathrm{a}}$ & $l_{\text {calc. }}$ & $l_{\text {exp. }}$ & $\sigma_{\mathrm{LS}}$ & $\sigma_{\exp }$ & $\left(r_{\mathrm{e}}-r_{\mathrm{a}}\right)$ & Group \\
\hline C6 & H11 & 3.258214 & 0.092800 & 0.091880 & $1.4 \mathrm{e}-03$ & $7.0 \mathrm{e}-03$ & -0.017100 & 105 \\
\hline $\mathrm{C} 1$ & H10 & 3.262542 & 0.092800 & 0.091880 & $1.4 \mathrm{e}-03$ & $7.0 \mathrm{e}-03$ & -0.017100 & 105 \\
\hline $\mathrm{C} 4$ & H12 & 3.264010 & 0.092700 & 0.091781 & $1.4 \mathrm{e}-03$ & $7.0 \mathrm{e}-03$ & -0.016200 & 105 \\
\hline $\mathrm{C} 1$ & H14 & 3.326688 & 0.098400 & 0.097425 & $1.5 \mathrm{e}-03$ & $7.4 \mathrm{e}-03$ & -0.001100 & 105 \\
\hline N2 & H12 & 3.373170 & 0.093600 & 0.092672 & $1.4 \mathrm{e}-03$ & $7.1 \mathrm{e}-03$ & -0.016100 & 105 \\
\hline N2 & H11 & 3.378898 & 0.093500 & 0.092573 & $1.4 \mathrm{e}-03$ & $7.1 \mathrm{e}-03$ & -0.015600 & 105 \\
\hline N5 & H10 & 3.386811 & 0.093600 & 0.092672 & $1.4 \mathrm{e}-03$ & $7.1 \mathrm{e}-03$ & -0.015200 & 105 \\
\hline $\mathrm{N} 2$ & O9 & 3.554974 & 0.064000 & 0.065341 & $9.3 \mathrm{e}-04$ & $2.0 \mathrm{e}-03$ & 0.001800 & 106 \\
\hline C3 & H13 & 3.585796 & 0.196800 & 0.200923 & $2.9 \mathrm{e}-03$ & $6.2 \mathrm{e}-03$ & -0.041200 & 106 \\
\hline C3 & $\mathrm{C} 7$ & 3.667660 & 0.065300 & 0.066668 & $9.5 \mathrm{e}-04$ & $2.1 \mathrm{e}-03$ & -0.012900 & 106 \\
\hline C6 & N8 & 3.694096 & 0.069700 & 0.071160 & $1.0 \mathrm{e}-03$ & $2.2 \mathrm{e}-03$ & -0.015800 & 106 \\
\hline $\mathrm{N} 2$ & H14 & 3.703567 & 0.129100 & 0.131805 & $1.9 \mathrm{e}-03$ & $4.1 \mathrm{e}-03$ & -0.013400 & 106 \\
\hline $\mathrm{C} 1$ & H11 & 3.760857 & 0.092300 & 0.094234 & $1.3 \mathrm{e}-03$ & $2.9 \mathrm{e}-03$ & -0.018300 & 106 \\
\hline C3 & H12 & 3.761074 & 0.092300 & 0.094234 & $1.3 \mathrm{e}-03$ & $2.9 \mathrm{e}-03$ & -0.017700 & 106 \\
\hline C6 & H10 & 3.763103 & 0.092500 & 0.094438 & $1.3 \mathrm{e}-03$ & $2.9 \mathrm{e}-03$ & -0.017600 & 106 \\
\hline N5 & $\mathrm{C} 7$ & 3.768580 & 0.067600 & 0.069016 & $9.8 \mathrm{e}-04$ & $2.1 \mathrm{e}-03$ & -0.011800 & 106 \\
\hline C6 & H13 & 3.900838 & 0.151600 & 0.154001 & $2.3 \mathrm{e}-03$ & $5.4 \mathrm{e}-03$ & -0.005300 & 107 \\
\hline H10 & H13 & 4.015149 & 0.230000 & 0.233643 & $3.4 \mathrm{e}-03$ & $8.2 \mathrm{e}-03$ & -0.055900 & 107 \\
\hline C3 & N8 & 4.061330 & 0.104500 & 0.106155 & $1.6 \mathrm{e}-03$ & $3.7 \mathrm{e}-03$ & -0.035000 & 107 \\
\hline N8 & H12 & 4.036902 & 0.139000 & 0.141202 & 2.1e-03 & $5.0 \mathrm{e}-03$ & -0.013800 & 107 \\
\hline H11 & H12 & 4.154446 & 0.127500 & 0.129520 & $1.9 \mathrm{e}-03$ & $4.5 \mathrm{e}-03$ & -0.019600 & 107 \\
\hline $\mathrm{C} 4$ & $\mathrm{C} 7$ & 4.179301 & 0.067900 & 0.068976 & $1.0 \mathrm{e}-03$ & $2.4 \mathrm{e}-03$ & -0.013700 & 107 \\
\hline N5 & O9 & 4.187309 & 0.106500 & 0.108187 & $1.6 \mathrm{e}-03$ & $3.8 \mathrm{e}-03$ & -0.014800 & 107 \\
\hline H12 & H13 & 4.471660 & 0.173600 & 0.175180 & $2.6 \mathrm{e}-03$ & $7.9 \mathrm{e}-03$ & -0.000900 & 108 \\
\hline $\mathrm{C} 7$ & H10 & 4.529056 & 0.108900 & 0.109891 & $1.6 \mathrm{e}-03$ & $5.0 \mathrm{e}-03$ & -0.016500 & 108 \\
\hline C6 & H14 & 4.514019 & 0.109900 & 0.110900 & $1.7 \mathrm{e}-03$ & $5.0 \mathrm{e}-03$ & 0.008200 & 108 \\
\hline N8 & H10 & 4.676184 & 0.155400 & 0.156814 & $2.3 \mathrm{e}-03$ & 7.1e-03 & -0.041800 & 108 \\
\hline C3 & O9 & 4.647787 & 0.071600 & 0.072252 & $1.1 \mathrm{e}-03$ & $3.3 \mathrm{e}-03$ & -0.001000 & 108 \\
\hline $\mathrm{C} 4$ & H13 & 4.657328 & 0.188100 & 0.189812 & $2.8 \mathrm{e}-03$ & $8.6 \mathrm{e}-03$ & -0.026200 & 108 \\
\hline H12 & H14 & 4.699375 & 0.180000 & 0.181638 & $2.7 \mathrm{e}-03$ & $8.2 \mathrm{e}-03$ & 0.008400 & 108 \\
\hline N5 & N8 & 4.806020 & 0.075100 & 0.075783 & $1.1 \mathrm{e}-03$ & $3.4 \mathrm{e}-03$ & -0.014900 & 108 \\
\hline N5 & H13 & 4.813913 & 0.170100 & 0.171648 & $2.6 \mathrm{e}-03$ & $7.7 \mathrm{e}-03$ & -0.008400 & 108 \\
\hline H10 & H12 & 4.847829 & 0.116700 & 0.117762 & $1.8 \mathrm{e}-03$ & $5.3 \mathrm{e}-03$ & -0.022400 & 108 \\
\hline $\mathrm{C} 4$ & O9 & 4.897708 & 0.091700 & 0.092534 & $1.4 \mathrm{e}-03$ & $4.2 \mathrm{e}-03$ & -0.007600 & 108 \\
\hline $\mathrm{C} 4$ & N8 & 4.921136 & 0.090300 & 0.091122 & $1.4 \mathrm{e}-03$ & $4.1 \mathrm{e}-03$ & -0.025800 & 108 \\
\hline C3 & H14 & 5.034633 & 0.132600 & 0.133807 & $2.0 \mathrm{e}-03$ & $6.0 \mathrm{e}-03$ & -0.007900 & 108 \\
\hline $\mathrm{C} 7$ & H11 & 5.266007 & 0.098700 & 0.098979 & $1.5 \mathrm{e}-03$ & $2.8 \mathrm{e}-02$ & -0.017800 & 109 \\
\hline O9 & H10 & 5.589838 & 0.106600 & 0.106901 & $1.7 \mathrm{e}-03$ & $3.0 \mathrm{e}-02$ & -0.000500 & 109 \\
\hline H10 & H14 & 5.621283 & 0.186100 & 0.186625 & $2.9 \mathrm{e}-03$ & $5.2 \mathrm{e}-02$ & -0.014700 & 109 \\
\hline H11 & H13 & 5.654181 & 0.208900 & 0.209490 & $3.3 \mathrm{e}-03$ & $5.8 \mathrm{e}-02$ & -0.032300 & 109 \\
\hline N5 & H14 & 5.693567 & 0.106400 & 0.106700 & $1.7 \mathrm{e}-03$ & $3.0 \mathrm{e}-02$ & 0.012000 & 109 \\
\hline $\mathrm{C} 4$ & H14 & 5.880578 & 0.114300 & 0.114623 & $1.8 \mathrm{e}-03$ & $3.2 \mathrm{e}-02$ & 0.002400 & 109 \\
\hline O9 & H11 & 5.962515 & 0.120200 & 0.120539 & $1.9 \mathrm{e}-03$ & $3.4 \mathrm{e}-02$ & -0.010200 & 109 \\
\hline N8 & H11 & 5.977860 & 0.120100 & 0.120439 & $1.9 \mathrm{e}-03$ & $3.4 \mathrm{e}-02$ & -0.028900 & 109 \\
\hline H11 & H14 & 6.941465 & 0.139300 & 0.139693 & $2.2 \mathrm{e}-03$ & $3.9 \mathrm{e}-02$ & 0.001100 & 109 \\
\hline
\end{tabular}


Table S16: GED terms (in $\AA$ ) for the refinement of the ISUCT GED data. Molecular intensities $s M(s)$ were averaged in the refinement procedure.

\begin{tabular}{|c|c|c|c|c|c|c|c|c|}
\hline Atom 1 & Atom 2 & $r_{\mathrm{a}}$ & $l_{\text {calc. }}$ & $l_{\text {exp. }}$ & $\sigma_{\mathrm{LS}}$ & $\sigma_{\exp }$ & $\left(r_{\mathrm{e}}-r_{\mathrm{a}}\right)$ & Group \\
\hline N8 & H14 & 1.017364 & 0.070100 & 0.078201 & $2.8 \mathrm{e}-03$ & $5.2 \mathrm{e}-03$ & -0.016500 & 100 \\
\hline N8 & H13 & 1.017132 & 0.070300 & 0.078424 & $2.8 \mathrm{e}-03$ & $5.2 \mathrm{e}-03$ & -0.016500 & 100 \\
\hline C6 & H12 & 1.099476 & 0.075300 & 0.084002 & $3.0 \mathrm{e}-03$ & $5.6 \mathrm{e}-03$ & -0.015800 & 100 \\
\hline C3 & H10 & 1.101344 & 0.075700 & 0.084448 & $3.0 \mathrm{e}-03$ & $5.7 \mathrm{e}-03$ & -0.015900 & 100 \\
\hline $\mathrm{C} 4$ & H11 & 1.102108 & 0.075700 & 0.084448 & $3.0 \mathrm{e}-03$ & $5.7 \mathrm{e}-03$ & -0.015900 & 100 \\
\hline $\mathrm{C} 7$ & O9 & 1.216620 & 0.038100 & 0.037996 & $1.1 \mathrm{e}-03$ & $1.2 \mathrm{e}-03$ & -0.002500 & 101 \\
\hline N2 & C3 & 1.336272 & 0.044200 & 0.044079 & $1.3 \mathrm{e}-03$ & $1.4 \mathrm{e}-03$ & -0.006000 & 101 \\
\hline N5 & C6 & 1.335639 & 0.044200 & 0.044079 & $1.3 \mathrm{e}-03$ & $1.4 \mathrm{e}-03$ & -0.005500 & 101 \\
\hline $\mathrm{C} 4$ & N5 & 1.340396 & 0.044400 & 0.044278 & $1.3 \mathrm{e}-03$ & $1.4 \mathrm{e}-03$ & -0.006000 & 101 \\
\hline $\mathrm{C} 1$ & $\mathrm{~N} 2$ & 1.340356 & 0.044600 & 0.044478 & $1.3 \mathrm{e}-03$ & $1.4 \mathrm{e}-03$ & -0.005500 & 101 \\
\hline $\mathrm{C} 7$ & N8 & 1.364174 & 0.044000 & 0.043879 & $1.3 \mathrm{e}-03$ & $1.4 \mathrm{e}-03$ & -0.015900 & 101 \\
\hline C3 & $\mathrm{C} 4$ & 1.398112 & 0.046300 & 0.046173 & $1.3 \mathrm{e}-03$ & $1.5 \mathrm{e}-03$ & -0.007400 & 101 \\
\hline $\mathrm{C} 1$ & C6 & 1.399757 & 0.046400 & 0.046273 & $1.3 \mathrm{e}-03$ & $1.5 \mathrm{e}-03$ & -0.007800 & 101 \\
\hline $\mathrm{C} 1$ & $\mathrm{C} 7$ & 1.505539 & 0.051200 & 0.051060 & $1.5 \mathrm{e}-03$ & $1.6 \mathrm{e}-03$ & -0.008800 & 101 \\
\hline H13 & H14 & 1.742487 & 0.115800 & 0.107534 & $4.7 \mathrm{e}-03$ & $8.8 \mathrm{e}-03$ & 0.003700 & 102 \\
\hline $\mathrm{C} 7$ & H14 & 2.028791 & 0.101600 & 0.094348 & $4.1 \mathrm{e}-03$ & $7.7 \mathrm{e}-03$ & 0.003700 & 102 \\
\hline $\mathrm{C} 7$ & H13 & 2.037307 & 0.100500 & 0.093326 & $4.1 \mathrm{e}-03$ & $7.6 \mathrm{e}-03$ & -0.001300 & 102 \\
\hline $\mathrm{N} 2$ & H10 & 2.079705 & 0.095700 & 0.088869 & $3.9 \mathrm{e}-03$ & $7.3 \mathrm{e}-03$ & -0.013400 & 102 \\
\hline N5 & H11 & 2.082221 & 0.095800 & 0.088962 & $3.9 \mathrm{e}-03$ & $7.3 \mathrm{e}-03$ & -0.013700 & 102 \\
\hline N5 & H12 & 2.088453 & 0.096000 & 0.089148 & $3.9 \mathrm{e}-03$ & 7.3e-03 & -0.011500 & 102 \\
\hline C1 & H12 & 2.156951 & 0.097800 & 0.090819 & $4.0 \mathrm{e}-03$ & $7.4 \mathrm{e}-03$ & -0.017300 & 102 \\
\hline C3 & H11 & 2.171237 & 0.097500 & 0.090540 & $4.0 \mathrm{e}-03$ & $7.4 \mathrm{e}-03$ & -0.015700 & 102 \\
\hline $\mathrm{C} 4$ & H10 & 2.174343 & 0.097400 & 0.090448 & $3.9 \mathrm{e}-03$ & $7.4 \mathrm{e}-03$ & -0.015500 & 102 \\
\hline $\mathrm{C} 4$ & C6 & 2.261810 & 0.052900 & 0.053362 & $1.4 \mathrm{e}-03$ & $1.6 \mathrm{e}-03$ & -0.010400 & 103 \\
\hline $\mathrm{C} 1$ & C3 & 2.270322 & 0.052800 & 0.053261 & $1.4 \mathrm{e}-03$ & $1.6 \mathrm{e}-03$ & -0.010400 & 103 \\
\hline N8 & O9 & 2.279347 & 0.053700 & 0.054169 & $1.5 \mathrm{e}-03$ & $1.6 \mathrm{e}-03$ & -0.011000 & 103 \\
\hline $\mathrm{N} 2$ & H13 & 2.310204 & 0.184500 & 0.186112 & $5.0 \mathrm{e}-03$ & $5.6 \mathrm{e}-03$ & -0.040600 & 103 \\
\hline N2 & $\mathrm{C} 4$ & 2.386935 & 0.053300 & 0.053766 & $1.5 \mathrm{e}-03$ & $1.6 \mathrm{e}-03$ & -0.008000 & 103 \\
\hline $\mathrm{N} 2$ & C6 & 2.389933 & 0.053500 & 0.053967 & $1.5 \mathrm{e}-03$ & $1.6 \mathrm{e}-03$ & -0.007600 & 103 \\
\hline $\mathrm{C} 1$ & N5 & 2.395040 & 0.053300 & 0.053766 & $1.5 \mathrm{e}-03$ & $1.6 \mathrm{e}-03$ & -0.007800 & 103 \\
\hline C3 & N5 & 2.396598 & 0.053400 & 0.053866 & $1.5 \mathrm{e}-03$ & $1.6 \mathrm{e}-03$ & -0.007600 & 103 \\
\hline $\mathrm{C} 1$ & O9 & 2.373247 & 0.060800 & 0.061331 & $1.7 \mathrm{e}-03$ & $1.8 \mathrm{e}-03$ & -0.006100 & 103 \\
\hline $\mathrm{C} 1$ & N8 & 2.414023 & 0.062700 & 0.063248 & $1.7 \mathrm{e}-03$ & $1.9 \mathrm{e}-03$ & -0.023900 & 103 \\
\hline N2 & $\mathrm{C} 7$ & 2.447962 & 0.062900 & 0.063449 & $1.7 \mathrm{e}-03$ & $1.9 \mathrm{e}-03$ & -0.010000 & 103 \\
\hline H10 & H11 & 2.516631 & 0.156600 & 0.157968 & $4.3 \mathrm{e}-03$ & $4.7 \mathrm{e}-03$ & -0.018300 & 103 \\
\hline C6 & $\mathrm{C} 7$ & 2.509145 & 0.066900 & 0.067484 & $1.8 \mathrm{e}-03$ & $2.0 \mathrm{e}-03$ & -0.013300 & 103 \\
\hline $\mathrm{C} 1$ & H13 & 2.516887 & 0.148500 & 0.149797 & $4.0 \mathrm{e}-03$ & $4.5 \mathrm{e}-03$ & -0.014000 & 103 \\
\hline O9 & H14 & 2.511915 & 0.141500 & 0.142736 & $3.9 \mathrm{e}-03$ & $4.3 \mathrm{e}-03$ & 0.013200 & 103 \\
\hline O9 & H12 & 2.539337 & 0.177300 & 0.178849 & $4.8 \mathrm{e}-03$ & $5.3 \mathrm{e}-03$ & -0.037000 & 103 \\
\hline $\mathrm{C} 1$ & $\mathrm{C} 4$ & 2.661451 & 0.058100 & 0.060555 & $2.1 \mathrm{e}-03$ & $2.8 \mathrm{e}-03$ & -0.011300 & 104 \\
\hline C3 & C6 & 2.661048 & 0.058600 & 0.061077 & $2.1 \mathrm{e}-03$ & $2.8 \mathrm{e}-03$ & -0.010700 & 104 \\
\hline $\mathrm{N} 2$ & N8 & 2.729261 & 0.097600 & 0.101725 & $3.5 \mathrm{e}-03$ & $4.7 \mathrm{e}-03$ & -0.037200 & 104 \\
\hline $\mathrm{C} 7$ & H12 & 2.699843 & 0.138500 & 0.144353 & $5.0 \mathrm{e}-03$ & $6.6 \mathrm{e}-03$ & -0.020600 & 104 \\
\hline $\mathrm{N} 2$ & N5 & 2.814919 & 0.062000 & 0.064620 & $2.2 \mathrm{e}-03$ & $3.0 \mathrm{e}-03$ & -0.004700 & 104 \\
\hline C6 & O9 & 2.837711 & 0.101100 & 0.105373 & $3.7 \mathrm{e}-03$ & $4.8 \mathrm{e}-03$ & -0.019200 & 104 \\
\hline O9 & H13 & 3.138754 & 0.094700 & 0.095581 & $4.1 \mathrm{e}-03$ & $1.4 \mathrm{e}-02$ & 0.006600 & 105 \\
\hline
\end{tabular}


Table S16 - continued from previous page

\begin{tabular}{|c|c|c|c|c|c|c|c|c|}
\hline Atom 1 & Atom 2 & $r_{\mathrm{a}}$ & $l_{\text {calc. }}$ & $l_{\text {exp. }}$ & $\sigma_{\mathrm{LS}}$ & $\sigma_{\exp }$ & $\left(r_{\mathrm{e}}-r_{\mathrm{a}}\right)$ & Group \\
\hline C6 & H11 & 3.250670 & 0.092500 & 0.093361 & $4.0 \mathrm{e}-03$ & $1.3 \mathrm{e}-02$ & -0.017200 & 105 \\
\hline $\mathrm{C} 1$ & H10 & 3.258143 & 0.092500 & 0.093361 & $4.0 \mathrm{e}-03$ & $1.3 \mathrm{e}-02$ & -0.017100 & 105 \\
\hline $\mathrm{C} 4$ & H12 & 3.256877 & 0.092500 & 0.093361 & $4.0 \mathrm{e}-03$ & $1.3 \mathrm{e}-02$ & -0.016300 & 105 \\
\hline $\mathrm{C} 1$ & H14 & 3.320299 & 0.097200 & 0.098105 & $4.2 \mathrm{e}-03$ & $1.4 \mathrm{e}-02$ & -0.002000 & 105 \\
\hline N2 & H12 & 3.363737 & 0.093300 & 0.094168 & $4.0 \mathrm{e}-03$ & $1.4 \mathrm{e}-02$ & -0.016200 & 105 \\
\hline N2 & H11 & 3.371381 & 0.093200 & 0.094067 & $4.0 \mathrm{e}-03$ & $1.4 \mathrm{e}-02$ & -0.015700 & 105 \\
\hline N5 & H10 & 3.381234 & 0.093300 & 0.094168 & $4.0 \mathrm{e}-03$ & $1.4 \mathrm{e}-02$ & -0.015300 & 105 \\
\hline N2 & O9 & 3.540878 & 0.062700 & 0.062659 & $2.5 \mathrm{e}-03$ & $4.5 \mathrm{e}-03$ & 0.001000 & 106 \\
\hline C3 & H13 & 3.589515 & 0.191800 & 0.191675 & 7.6e-03 & $1.4 \mathrm{e}-02$ & -0.038800 & 106 \\
\hline C3 & $\mathrm{C} 7$ & 3.652384 & 0.064200 & 0.064158 & $2.5 \mathrm{e}-03$ & $4.6 \mathrm{e}-03$ & -0.012800 & 106 \\
\hline C6 & N8 & 3.681649 & 0.068200 & 0.068156 & $2.7 \mathrm{e}-03$ & $4.9 \mathrm{e}-03$ & -0.015700 & 106 \\
\hline N2 & H14 & 3.704880 & 0.125800 & 0.125718 & $5.0 \mathrm{e}-03$ & $9.0 \mathrm{e}-03$ & -0.013400 & 106 \\
\hline $\mathrm{C} 1$ & H11 & 3.754539 & 0.091800 & 0.091740 & $3.6 \mathrm{e}-03$ & 6.6e-03 & -0.018300 & 106 \\
\hline C3 & H12 & 3.751140 & 0.091800 & 0.091740 & $3.6 \mathrm{e}-03$ & $6.6 \mathrm{e}-03$ & -0.017800 & 106 \\
\hline C6 & H10 & 3.753477 & 0.092000 & 0.091940 & $3.6 \mathrm{e}-03$ & $6.6 \mathrm{e}-03$ & -0.017800 & 106 \\
\hline N5 & $\mathrm{C} 7$ & 3.749005 & 0.066300 & 0.066257 & $2.6 \mathrm{e}-03$ & $4.7 \mathrm{e}-03$ & -0.011800 & 106 \\
\hline C6 & H13 & 3.895700 & 0.149100 & 0.146970 & $5.7 \mathrm{e}-03$ & $9.9 \mathrm{e}-03$ & -0.005900 & 107 \\
\hline H10 & H13 & 4.022408 & 0.224700 & 0.221490 & $8.6 \mathrm{e}-03$ & $1.5 \mathrm{e}-02$ & -0.052700 & 107 \\
\hline C3 & N8 & 4.055428 & 0.101300 & 0.099853 & $3.9 \mathrm{e}-03$ & $6.7 \mathrm{e}-03$ & -0.033400 & 107 \\
\hline N8 & H12 & 4.018943 & 0.137000 & 0.135043 & $5.3 \mathrm{e}-03$ & $9.1 \mathrm{e}-03$ & -0.014200 & 107 \\
\hline H11 & H12 & 4.151169 & 0.127300 & 0.125482 & $4.9 \mathrm{e}-03$ & $8.5 \mathrm{e}-03$ & -0.019800 & 107 \\
\hline $\mathrm{C} 4$ & $\mathrm{C} 7$ & 4.159789 & 0.066700 & 0.065747 & $2.6 \mathrm{e}-03$ & $4.4 \mathrm{e}-03$ & -0.013700 & 107 \\
\hline N5 & O9 & 4.163216 & 0.103200 & 0.101726 & $4.0 \mathrm{e}-03$ & $6.9 \mathrm{e}-03$ & -0.014600 & 107 \\
\hline H12 & H13 & 4.462024 & 0.171500 & 0.179375 & $6.9 \mathrm{e}-03$ & $1.5 \mathrm{e}-02$ & -0.002200 & 108 \\
\hline $\mathrm{C} 7$ & H10 & 4.518647 & 0.108000 & 0.112959 & $4.4 \mathrm{e}-03$ & $9.6 \mathrm{e}-03$ & -0.016600 & 108 \\
\hline C6 & H14 & 4.500303 & 0.108500 & 0.113482 & $4.4 \mathrm{e}-03$ & $9.6 \mathrm{e}-03$ & 0.006400 & 108 \\
\hline N8 & H10 & 4.675366 & 0.152100 & 0.159084 & $6.1 \mathrm{e}-03$ & $1.3 \mathrm{e}-02$ & -0.040000 & 108 \\
\hline C3 & O9 & 4.627027 & 0.070000 & 0.073214 & $2.8 \mathrm{e}-03$ & $6.2 \mathrm{e}-03$ & -0.001900 & 108 \\
\hline $\mathrm{C} 4$ & H13 & 4.655780 & 0.183700 & 0.192135 & $7.4 \mathrm{e}-03$ & $1.6 \mathrm{e}-02$ & -0.025300 & 108 \\
\hline H12 & H14 & 4.677544 & 0.177500 & 0.185650 & $7.2 \mathrm{e}-03$ & $1.6 \mathrm{e}-02$ & 0.006400 & 108 \\
\hline N5 & N8 & 4.791939 & 0.073300 & 0.076666 & $3.0 \mathrm{e}-03$ & $6.5 \mathrm{e}-03$ & -0.015000 & 108 \\
\hline N5 & H13 & 4.809715 & 0.166700 & 0.174355 & $6.7 \mathrm{e}-03$ & $1.5 \mathrm{e}-02$ & -0.008800 & 108 \\
\hline H10 & H12 & 4.841573 & 0.116300 & 0.121640 & $4.7 \mathrm{e}-03$ & $1.0 \mathrm{e}-02$ & -0.022800 & 108 \\
\hline $\mathrm{C} 4$ & O9 & 4.873419 & 0.089100 & 0.093191 & $3.6 \mathrm{e}-03$ & $7.9 \mathrm{e}-03$ & -0.008200 & 108 \\
\hline $\mathrm{C} 4$ & N8 & 4.909499 & 0.087800 & 0.091832 & $3.5 \mathrm{e}-03$ & $7.8 \mathrm{e}-03$ & -0.025200 & 108 \\
\hline C3 & H14 & 5.030211 & 0.129200 & 0.135133 & $5.2 \mathrm{e}-03$ & $1.1 \mathrm{e}-02$ & -0.008500 & 108 \\
\hline $\mathrm{C} 7$ & H11 & 5.250493 & 0.097700 & 0.098020 & $4.4 \mathrm{e}-03$ & $4.7 \mathrm{e}-02$ & -0.018200 & 109 \\
\hline O9 & H10 & 5.574134 & 0.105500 & 0.105846 & $4.7 \mathrm{e}-03$ & $5.1 \mathrm{e}-02$ & -0.002000 & 109 \\
\hline H10 & H14 & 5.623254 & 0.182100 & 0.182697 & $8.2 \mathrm{e}-03$ & $8.7 \mathrm{e}-02$ & -0.015200 & 109 \\
\hline H11 & H13 & 5.656841 & 0.204400 & 0.205070 & $9.2 \mathrm{e}-03$ & $9.8 \mathrm{e}-02$ & -0.031600 & 109 \\
\hline N5 & H14 & 5.678144 & 0.104900 & 0.105244 & $4.7 \mathrm{e}-03$ & $5.0 \mathrm{e}-02$ & 0.009700 & 109 \\
\hline $\mathrm{C} 4$ & H14 & 5.869097 & 0.112000 & 0.112367 & $5.0 \mathrm{e}-03$ & $5.4 \mathrm{e}-02$ & 0.000700 & 109 \\
\hline O9 & H11 & 5.942073 & 0.117800 & 0.118186 & $5.3 \mathrm{e}-03$ & $5.6 \mathrm{e}-02$ & -0.011200 & 109 \\
\hline N8 & H11 & 5.970578 & 0.117800 & 0.118186 & $5.3 \mathrm{e}-03$ & $5.6 \mathrm{e}-02$ & -0.028700 & 109 \\
\hline H11 & H14 & 6.934657 & 0.137100 & 0.137550 & $6.1 \mathrm{e}-03$ & $6.6 \mathrm{e}-02$ & -0.001100 & 109 \\
\hline
\end{tabular}


Table S17: Equilibrium Cartesian coordinates for PZA structure refined from UBi GED data $\left(\alpha=3.0 \cdot 10^{5}\right)$.

\begin{tabular}{cccc}
\hline Atom & $\mathrm{x}$ & $\mathrm{y}$ & $\mathrm{z}$ \\
\hline $\mathrm{C}$ & 0.1793956940 & 0.0131822262 & 0.0000000001 \\
$\mathrm{~N}$ & -0.3860090687 & -1.1936597492 & 0.0000000006 \\
$\mathrm{C}$ & -1.7146658956 & -1.2155584710 & 0.0000000008 \\
$\mathrm{C}$ & -2.4711072877 & -0.0479344462 & 0.0000000005 \\
$\mathrm{~N}$ & -1.9157512150 & 1.1634415446 & -0.0000000000 \\
$\mathrm{C}$ & -0.5832543525 & 1.1843814658 & -0.0000000002 \\
$\mathrm{C}$ & 1.6785170943 & 0.0961655698 & -0.0000000001 \\
$\mathrm{~N}$ & 2.2870343513 & -1.1058156534 & 0.0000000003 \\
$\mathrm{O}$ & 2.2482178629 & 1.1749154912 & -0.0000000005 \\
$\mathrm{H}$ & -2.1943141722 & -2.1875112133 & -0.0000000191 \\
$\mathrm{H}$ & -3.5544598603 & -0.0939494963 & 0.0000000006 \\
$\mathrm{H}$ & -0.0847268077 & 2.1448702536 & -0.0000000006 \\
$\mathrm{H}$ & 1.7277261194 & -1.9415634485 & 0.0000000006 \\
$\mathrm{H}$ & 3.2916775789 & -1.1441741955 & 0.0000000002 \\
\hline
\end{tabular}

Table S18: Equilibrium Cartesian coordinates for PZA structure refined from LMSU GED data $(\alpha=$ $\left.3.0 \cdot 10^{6}\right)$.

\begin{tabular}{cccc}
\hline Atom & $\mathrm{x}$ & $\mathrm{y}$ & $\mathrm{z}$ \\
\hline $\mathrm{C}$ & 0.1788297320 & 0.0102290474 & 0.0000000001 \\
$\mathrm{~N}$ & -0.3906775925 & -1.2014229129 & 0.0000000006 \\
$\mathrm{C}$ & -1.7271341569 & -1.2189743565 & 0.0000000008 \\
$\mathrm{C}$ & -2.4806973035 & -0.0429072096 & 0.0000000005 \\
$\mathrm{~N}$ & -1.9173354682 & 1.1711060039 & -0.0000000000 \\
$\mathrm{C}$ & -0.5796568620 & 1.1847165531 & -0.0000000002 \\
$\mathrm{C}$ & 1.6825606034 & 0.0967966726 & -0.0000000001 \\
$\mathrm{~N}$ & 2.2976328411 & -1.1068032685 & 0.0000000003 \\
$\mathrm{O}$ & 2.2551713890 & 1.1755767722 & -0.0000000005 \\
$\mathrm{H}$ & -2.2088956039 & -2.1878754284 & -0.0000000191 \\
$\mathrm{H}$ & -3.5625659533 & -0.0829879165 & 0.0000000006 \\
$\mathrm{H}$ & -0.0767202218 & 2.1409340931 & -0.0000000006 \\
$\mathrm{H}$ & 1.7427628352 & -1.9428358950 & 0.0000000006 \\
$\mathrm{H}$ & 3.2989863048 & -1.1404916045 & 0.0000000002 \\
\hline
\end{tabular}


Table S19: Equilibrium Cartesian coordinates for PZA structure refined from ISUCT GED data $(\alpha=$ $\left.5.0 \cdot 10^{4}\right)$.

\begin{tabular}{cccc}
\hline Atom & $\mathrm{x}$ & $\mathrm{y}$ & $\mathrm{z}$ \\
\hline $\mathrm{C}$ & 0.1787895321 & 0.0078135747 & 0.0000000001 \\
$\mathrm{~N}$ & -0.3922217615 & -1.1987462661 & 0.0000000006 \\
$\mathrm{C}$ & -1.7224072333 & -1.2139467137 & 0.0000000008 \\
$\mathrm{C}$ & -2.4708960768 & -0.0418354114 & 0.0000000005 \\
$\mathrm{~N}$ & -1.9076444827 & 1.1678593996 & -0.0000000000 \\
$\mathrm{C}$ & -0.5775329428 & 1.1763689636 & -0.0000000002 \\
$\mathrm{C}$ & 1.6728661963 & 0.0970428514 & -0.0000000001 \\
$\mathrm{~N}$ & 2.2979374658 & -1.0975819740 & 0.0000000003 \\
$\mathrm{O}$ & 2.2409708542 & 1.1700495180 & -0.0000000005 \\
$\mathrm{H}$ & -2.2081634351 & -2.1846317219 & -0.0000000191 \\
$\mathrm{H}$ & -3.5563862077 & -0.0813122752 & 0.0000000006 \\
$\mathrm{H}$ & -0.0686175695 & 2.1331123419 & -0.0000000006 \\
$\mathrm{H}$ & 1.7538352407 & -1.9373535098 & 0.0000000006 \\
$\mathrm{H}$ & 3.2984798014 & -1.1229570263 & 0.0000000002 \\
\hline
\end{tabular}

Table S20: Equilibrium Cartesian coordinates for PZA structure optimized at CCSD(T)/cc-pwCVTZ level of theory.

\begin{tabular}{cccc}
\hline Atom & $\mathrm{x}$ & $\mathrm{y}$ & $\mathrm{z}$ \\
\hline $\mathrm{C}$ & -0.176935535639 & 0.009313038031 & 0.000000000000 \\
$\mathrm{~N}$ & 0.392544096066 & -1.200531888597 & 0.000000000000 \\
$\mathrm{C}$ & 1.727200869188 & -1.215715523777 & 0.000000000000 \\
$\mathrm{C}$ & 2.475978887034 & -0.041369897262 & 0.000000000000 \\
$\mathrm{~N}$ & 1.910712808006 & 1.170066101310 & 0.000000000000 \\
$\mathrm{C}$ & 0.574887135260 & 1.183734585606 & 0.000000000000 \\
$\mathrm{C}$ & -1.679575207825 & 0.096049200306 & 0.000000000000 \\
$\mathrm{~N}$ & -2.294186414434 & -1.105951276868 & 0.000000000000 \\
$\mathrm{O}$ & -2.250544267107 & 1.172790141743 & 0.000000000000 \\
$\mathrm{H}$ & 2.210786571836 & -2.183751858704 & 0.000000000000 \\
$\mathrm{H}$ & 3.557628683011 & -0.079068743777 & 0.000000000000 \\
$\mathrm{H}$ & 0.072248220549 & 2.140107393581 & 0.000000000000 \\
$\mathrm{H}$ & -1.739504229643 & -1.942073714486 & 0.000000000000 \\
$\mathrm{H}$ & -3.295885571582 & -1.139621227107 & 0.000000000000 \\
\hline
\end{tabular}


Root-mean-square deviations (RMSD) and mean absolute deviations (MAD) have been calculated for all pairs of parameter sets as:

$$
\begin{aligned}
\operatorname{RMSD} & =\sqrt{\frac{\sum_{i=1}^{N}\left(p_{i}^{\mathrm{A}}-p_{i}^{\mathrm{B}}\right)^{2}}{N}} \\
\mathrm{MAD} & =\frac{\sum_{i=1}^{N}\left|p_{i}^{\mathrm{A}}-p_{i}^{\mathrm{B}}\right|}{N}
\end{aligned}
$$

where $p_{i}^{\mathrm{A}}$ and $p_{i}^{\mathrm{B}}$ are the values of $i$-th parameter from sets $\mathrm{A}$ and $\mathrm{B}$, respectively; $N$ is the total number of parameters.

Table S21: RMSD for bond lengths (A, lower triangle) and angles (degrees, upper triangle) in PZA

\begin{tabular}{ccccc}
\hline & CCSD $(\mathrm{T})$ & UBi & LMSU & ISUCT \\
CCSD $(\mathrm{T})$ & & 0.20 & 0.11 & 0.27 \\
$\mathrm{UBi}$ & 0.003 & & 0.15 & 0.30 \\
LMSU & 0.002 & 0.004 & & 0.20 \\
ISUCT & 0.004 & 0.003 & 0.005 & \\
\hline
\end{tabular}

Table S22: MAD for bond lengths ( $\AA$, lower triangle) and angles (degrees, upper triangle) in PZA

\begin{tabular}{ccccc}
\hline & CCSD $(\mathrm{T})$ & UBi & LMSU & ISUCT \\
CCSD $(\mathrm{T})$ & & 0.14 & 0.06 & 0.19 \\
$\mathrm{UBi}$ & 0.003 & & 0.11 & 0.21 \\
$\mathrm{LMSU}$ & 0.001 & 0.004 & & 0.15 \\
$\mathrm{ISUCT}$ & 0.003 & 0.003 & 0.005 & \\
\hline
\end{tabular}

Table S23: RMSD ( $\AA$, upper triangle) and MAD ( $\AA$, lower triangle) for amplitudes of interatomic vibrations in PZA

\begin{tabular}{ccccc}
\hline & B3LYP & UBi & LMSU & ISUCT \\
B3LYP & & 0.010 & 0.004 & 0.004 \\
UBi & 0.008 & & 0.007 & 0.009 \\
LMSU & 0.003 & 0.005 & & 0.004 \\
ISUCT & 0.003 & 0.007 & 0.003 & \\
\hline
\end{tabular}

\section{References}

[1] Tomáš Baše, Josef Holub, Jindřich Fanfrlík, Drahomír Hnyk, Paul D. Lane, Derek A. Wann, Yury V. Vishnevskiy, Denis Tikhonov, Christian G. Reuter, and Norbert W. Mitzel. Icosahedral carbaboranes with peripheral hydrogen-chalcogenide groups: Structures from gas electron diffraction and chemical shielding in solution. Chemistry - A European Journal, 25(9):2313-2321, 2019.

[2] Ana Borba, Merwe Albrecht, Andrea Gómez-Zavaglia, Martin A. Suhm, and Rui Fausto. Low temperature infrared spectroscopy study of pyrazinamide: From the isolated monomer to the stable low temperature crystalline phase. The Journal of Physical Chemistry A, 114(1):151-161, 2010. 